Mechanical Properties of Arteries

An In Vivo Parameter Identification Method

\title{
Jan-Lucas Gade
}

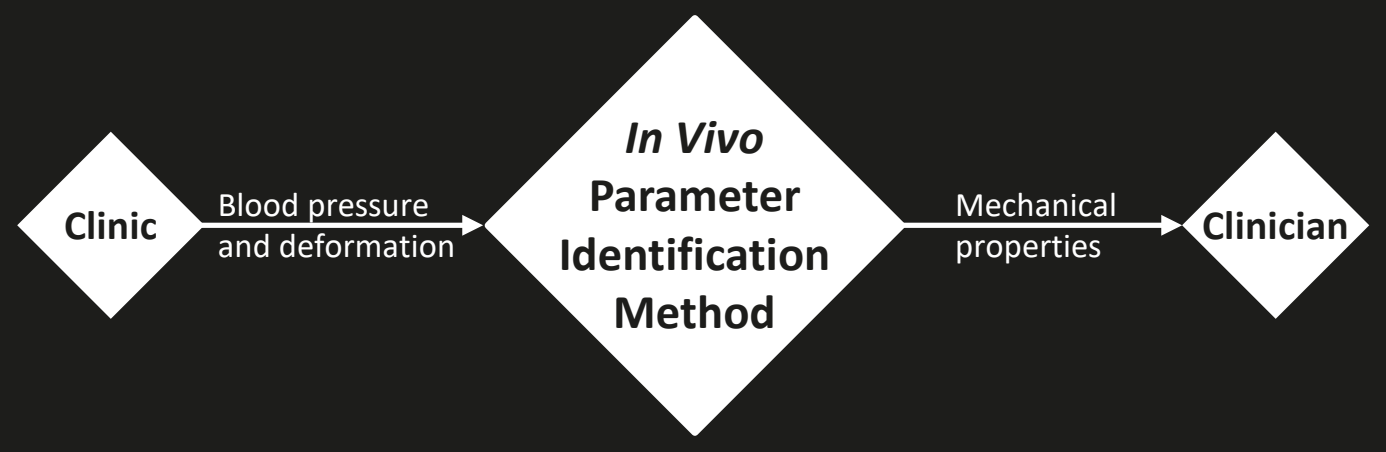



Linköping Studies in Science and Technology.

Dissertations, No. 2113

\title{
Mechanical Properties of Arteries An In Vivo Parameter Identification Method
}

\author{
Jan-Lucas Gade
}

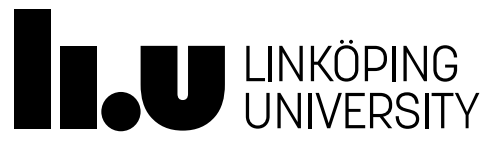

Solid Mechanics

Department of Management and Engineering

Linköping University

SE-581 83 Linköping, Sweden

Linköping, February 2021 
Cover:

Overall project divided into parts.

Printed by:

LiU-Tryck, Linköping, Sweden, 2021

ISBN: 978-91-7929-718-3

ISSN: 0345-7524

Distributed by:

Solid Mechanics

Department of Management and Engineering

Linköping University

SE-581 83 Linköping, Sweden

\section{(C) 2021 Jan-Lucas Gade}

This document was prepared with IATEX, January 22, 2021

No part of this publication may be reproduced, stored in a retrieval system, or be transmitted, in any form or by any means, electronic, mechanical, photocopying, recording, or otherwise, without prior permission of the author. 


\section{Preface}

The work presented in this dissertation has been created at the division of Solid Mechanics at Linköping University. The research has been financially supported by the Swedish Research Council, the graduate research school at the Department of Management and Engineering at Linköping University, and by Linköping University, the support of which is greatly acknowledged.

First and foremost, I would like to thank my main supervisor Professor Jonas Stålhand and my co-supervisor Associate Professor Carl-Johan Thore for their endless support. Throughout the project you always had an open door and guided me with your valuable comments, thoughts and ideas.

I would also like to take the opportunity and thank my research group: Jerker Karlsson, who I always consulted for medical input; and the late Professor Toste Länne, who invited me to present my work to the medical community and provided in vivo data.

Furthermore, I would like to thank my fellow Ph.D. colleagues for not only making the Ph.D. life a lot of fun but also many fruitful discussions. A special thank you goes to my former office mate and dear friend Dr. Christian Busse, without whom I would have not started this Ph.D. project.

Finally, I would like to express my gratitude to my family and especially my parents Regina and Rüdiger for their unconditional support and encouragement.

\section{Thank you!}

Linköping, December 2020

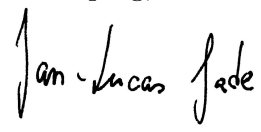

Jan-Lucas Gade 



\section{Abstract}

In this dissertation, a method is proposed that identifies the mechanical properties of arteries in vivo. The mechanical properties of an artery are linked to the development of cardiovascular diseases. The possibility to identify the mechanical properties of an artery inside the human body could, thus, facilitate disease diagnostization, treatment and monitoring.

Supplied with information obtainable in the clinic, typically limited to timeresolved pressure-radius measurement pairs, the proposed in vivo parameter identification method solves a non-convex minimization problem to determine parameters related to the mechanical properties of the blood vessel. The artery is treated as a homogeneous, incompressible, residual stress-free, thin-walled tube consisting of an elastin dominated matrix with embedded collagen fibers.

To validate the in vivo parameter identification method, in silico arteries in the form of finite element models are created using published data for the human abdominal aorta. With these in silico arteries which serve as mock experiments with pre-defined material parameters and boundary conditions, in vivo-like pressureradius data sets are generated. The mechanical properties of the in silico arteries are then determined using the proposed parameter identification method. By comparing the identified and the pre-defined parameters, the identification method is quantitatively validated and it is shown that the parameters agree well for healthy arteries. Furthermore, the identified parameters are used to compare the stress state in the arterial model and in the in silico arteries. The stress state is thereby decomposed into an isotropic and an anisotropic component which are primarily associated with the elastin dominated matrix and the collagen fibers, respectively. The comparison of the decomposed stress states shows a close agreement for arteries exhibiting a physiological stress gradient.

Another important aspect is the identification of parameters by solving a nonconvex minimization problem. The non-convexity of the problem implies that incorrect parameter values, corresponding to local minima, may be found when common gradient-based solution techniques are employed. A problem-specific global algorithm based on the branch-and-bound method is, therefore, created which ensures that the global minimum and accordingly the correct parameters are obtained. It turns out that the gradient-based solution technique identifies the correct parameters if certain requirements are met, among others the use of the heuristic multi-start method.

In a last step, the in vivo parameter identification method is extended to also identify parameters related to smooth muscle contraction. To prevent an 
overparameterization caused by the increased number of model parameters, the model is simultaneously fit to clinical data measured during three different arterial conditions: basal; constricted; and dilated. Despite the simple contraction model the extended method fits the clinical data well.

Finally, in this dissertation it is shown that the proposed in vivo parameter identification method identifies the mechanical properties of arteries well. An open question for future research is how this method can be applied in a clinical setting to facilitate cardiovascular disease diagnostization, treatment and monitoring. 


\section{Zusammenfassung}

In dieser Dissertation der Ingenieurwissenschaften wird eine Methode zur Identifikation der mechanischen Eigenschaften von Arterien in vivo vorgestellt. Die Ausbildung kardiovaskulärer Krankheiten ist mit den mechanischen Eigenschaften der Arterie verknüpft und deren Identifikation hat daher das Potenzial die Diagnose, die Behandlung und die Überwachung dieser Krankheiten zu verbessern.

Basierend auf klinisch möglichen Messungen, die üblicherweise auf ein zeitaufgelöstes Druck-Radiussignal limitiert sind, berechnet die Methode durch Lösen eines nicht-konvexen Minimierungsproblems Parameter, die die mechanischen Eigenschaften des Blutgefäßes darstellen. Die Arterie wird dabei als eine homogene, inkompressible, restspannungsfreie und dünnwandige Röhre beschrieben, deren Wand aus einer elastindominierten Matrix mit eingebetteten Kollagenfasern besteht.

Um die in vivo Parameteridentifikationsmethode zu validieren, werden virtuelle Arterien in Form von Finite Elemente Modellen erstellt. Diese in silico Arterien beruhen auf publizierten Materialparametern der menschlichen Abdominalaorta und dienen als Pseudoexperimente mit vordefinierten mechanischen Eigenschaften und Randbedingungen. Mit diesen Arterien werden in vivo ähnliche Druck-Radiussignale erstellt und anschließend werden ihre mechanischen Eigenschaften mit Hilfe der Parameteridentifikationsmethode bestimmt. Der Vergleich der berechneten und der vordefinierten Parameter ermöglicht die quantitative Validierung der Methode und es zeigt sich, dass im Falle gesunder Arterien die Parameter gut übereinstimmen. Weiterhin wird mit Hilfe der berechneten Parameter der Spannungszustand in dem Arterienmodell bestimmt und mit den in silico Arterien verglichen.

Der Spannungszustand ist dabei aufgeteilt in einen isotropen und einen anisotropen Anteil, welcher respektive mit der elastindomierten Matrix bzw. mit den Kollagenfasern verknüpft ist. Der Vergleich der Spannungszustände zeigt eine gute Übereinstimmung für Arterien mit einem physiologischen Spannungsgradienten in der Arterienwand.

Ein weiterer wichtiger Aspekt ist die Berechnung der Parameter durch das Lösen des nicht-konvexen Minimierungsproblems. Bei Verwendung gewöhnlicher Gradientenverfahren können infolge der Nicht-Konvexität Parameter berechnet werden, die zu einem lokalen Minimum gehören. Es wird daher ein auf der Branch-and-Bound Methode basierender Algorithmus entwickelt, welcher die Erkennung des globalen Minimums sicherstellt. Es zeigt sich, dass auch gewöhnliche Gradientenverfahren die richtigen Parameter berechnen, jedoch nur in Verbindung mit heuristischen Maßnahmen unter anderem der Multi-Start Methode.

In einem letzten Schritt wird die in vivo Parameteridentifikationsmethode 
erweitert um auch Parameter zu berechnen, die mit der Kontraktion der glatten Muskulatur in Verbindung stehen. Zur Vermeidung einer Überparametrisierung, die infolge der erhöhten Modellparameteranzahl entsteht, wird das Arterienmodell simultan mit drei klinischen Messungen abgestimmt. Die klinischen Messungen sind dabei zu unterschiedlichen arteriellen Zuständen erhoben worden: normal, verengt und geweitet. Trotz des einfachen Kontraktionsmodells spiegelt die erweiterte Methode die klinischen Messungen gut wieder.

Zusammenfassend lässt sich sagen, dass die in dieser Dissertation entwickelte in vivo Parameteridentifikationsmethode in der Lage ist, die mechanischen Eigenschaften einer Arterie zu bestimmen. Inwiefern diese Methode in der Klinik eingesetzt werden kann um die Diagnose, die Behandlung und die Überwachung von Herz-Kreislauferkrankungen zu verbessern, ist eine offene Frage und bedarf weiterer Forschung. 


\section{Sammanfattning}

I denna avhandling föreslås en metod för att identifiera mekaniska egenskaper hos artärer in vivo. Utvecklingen av hjärt-kärlsjukdomar är kopplad till förändringar i de mekaniska egenskaperna, och möjligheten att identifiera dessa förändringar skulle således underlätta diagnostisering, behandling och uppföljning.

Den förslagna metoden använder kliniskt mätbara tryck-radie-signaler och löser ett icke-konvex minimeringsproblem för att bestämma parametrar som beskriver kärlets mekaniska egenskaper. Artären modelleras som ett homogent, inkompressibelt och spänningsfritt tunnväggigt rör. Kärlväggen utgörs av en elastindominerad matris med inbäddade kollagenfibrer.

För att validera parameteridentifieringen skapas en uppsättning fysiologiskt representativa, virtuella artärer med hjälp av finita elementmetoden. Dessa in silico-artärer baseras på publicerade data för mänsklig bukaorta och används för att generera fiktiva tryck-radie-signaler, vilka sedan matas in i den förslagna metoden. Genom att parametrar och randvillkor för in silico-artärerna är kända kan resultatet från parameteridentifieringen direkt jämföras mot in silico-artärerna. Jämförelsen visar god överensstämmelse för parametrar som svarar mot friska kärl. De identifierade parametrarna har också använts för att jämföra spänningstillståndet i artärmodellen och in silico-artäreren. Spänningstillståndet har delats upp i en isotrop och en anisotrop komponent som svarar mot, i huvudsak, den elastindominerade matrisen samt kollagenfibrerna. Resultatet visar en mycket god överensstämmelse för bägge komponenterna när de jämförs mot in silico-artärer med fysiologisk spänningsgradient.

En annan viktig aspekt i arbetet är identifieringen av parametrar som sker genom att lösa ett icke-konvex minimeringsproblem. Problemets icke-konvexitet innebär att suboptimala parameterar, kopplade till lokala minima, kan identifieras när vanliga gradientbaserade tekniker används. En problemspecifik global algoritm baserad på branch-and-bound metoden har därför utvecklats för att säkerställa att det globala minimumet identifieras. Det visar sig att den gradientbaserade teknik som används i studien identifierar korrekta parametrarna under vissa förutsättningar, bland annat att en heuristisk multi-start-metod används.

I ett sista steg utökas metoden till att också identifiera parametrar kopplade till kontraktion av glatt muskulatur. För att förhindra en överparameterisering av modellen på grund av det ökade antalet parametrar används data från aorta i tre arteriella tillstånd: normal, kontraherad och dilaterad. Trots den enkla muskelmodellen visar metoden en mycket god överensstämmelse med mätdata.

Slutligen visas i denna avhandling att den föreslagna metoden för att identi- 
fiera mekaniska egenskaper hos artärer in vivo fungerar väl. En öppen fråga för framtida forskning är hur metoden kan tillämpas i en klinisk miljö för att underlätta diagnostisering, behandling och uppföljning av hjärt-kärlsjukdomar. 


\section{List of Papers}

In this dissertation, the following papers are included:

I. J.-L. Gade, J. Stålhand, C.-J. Thore (2019). An in vivo parameter identification method for arteries: numerical validation for the human abdominal aorta, Computer Methods in Biomechanics and Biomedical Engineering, Volume 22, Issue 4.

II. J.-L. Gade, J. Karlsson, C.-J. Thore, J. Stålhand (2020). Assessing the accuracy of the stress predicted by an in vivo parameter identification method for arteries, Submitted.

III. J.-L. Gade, C.-J. Thore, J. Stålhand (2020). Identification of mechanical properties of arteries with certification of global optimality, Submitted.

IV. J.-L. Gade, C.-J. Thore, B. Sonesson, J. Stålhand (2020). In vivo parameter identification in arteries considering multiple levels of smooth muscle activity, Submitted.

\section{Note}

The appended papers are reformatted to fit the layout of the thesis and the published appended papers are reprinted with the permission of the respective copyright holder.

\section{The author's contribution}

All authors contributed to the design and analysis in the papers above. Jan-Lucas Gade is responsible for all finite element and MATLAB implementations, and performed the numerical and statistical analyses in all studies. In addition, the manuscripts were prepared and submitted by him after they were critically reviewed by all authors. 



\section{Contents}

$\begin{array}{ll}\text { Preface } & \text { iii }\end{array}$

Abstract $\quad$ v

Zusammenfassung vii

Sammanfattning ix

List of Papers $\quad$ xi

Contents

Abbreviations and Glossary xvii

$\begin{array}{ll}\text { Part I - Summary of the Work } & 1\end{array}$

1 Introduction 3

1.1 Aim of the work . . . . . . . . . . . . . . . 5 5

1.2 Scope of the work . . . . . . . . . . . . . . . 5

1.3 Outline . . . . . . . . . . . . . . . . . . 6

2 Arteries $\quad 9$

2.1 Function and structure . . . . . . . . . . . . . . . . . 9

2.2 Mechanical properties . . . . . . . . . . . . . . . . 11

2.3 Smooth muscle cell contraction . . . . . . . . . . . . . 16

3 Continuum Mechanics $\quad 19$

3.1 Kinematics . . . . . . . . . . . . . . . . . . . . . . . . . . 19

3.2 Definition of stress . . . . . . . . . . . . . . . . . 20

3.3 Balance principles . . . . . . . . . . . . . . . . . . . 21

3.3 .1 Conservation of mass . . . . . . . . . . . . . . . . 21

3.3.2 Conservation of linear momentum . . . . . . . . . . . . . 22

3.3.3 Conservation of angular momentum . . . . . . . . . . . . 22

3.3.4 Conservation of energy . . . . . . . . . . . . . . . 23

3.3 .5 Entropy inequality . . . . . . . . . . . . . . . . . 24

3.4 Constitutive equation . . . . . . . . . . . . . . . 25 
3.4.1 Constitutive equation in terms of invariants . . . . . . . 26

3.4.2 Constitutive equation with directional properties . . . . . 26

3.5 Boundary conditions ... . . . . . . . . . . . 27

4 Mechanical Models for Arteries $\quad 29$

4.1 General continuum-mechanical model for arteries . . . . . . . . . . 29

4.1 .1 Kinematics . . . . . . . . . . . . . . . . . . . . . . . 29

4.1 .2 Constitutive equation . . . . . . . . . . . . . . . 31

4.1.2.1 Passive constitutive model . . . . . . . . . . . . . 32

4.1.2.2 Active constitutive model . . . . . . . . . . . 33

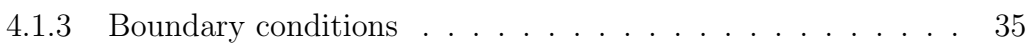

4.1 .4 Equilibrium . . . . . . . . . . . . . . . . . . . . . . 35

4.2 Constitutive membrane model . . . . . . . . . . . . . . 36

4.2 .1 Equilibrium stresses . . . . . . . . . . . . . . . . 37

4.2.2 Constitutively determined stresses . . . . . . . . . . . 38

5 Parameter Identification $\quad 41$

5.1 Normalization . . . . . . . . . . . . . . . . . . . . . 43

5.2 Solving the minimization problem numerically . . . . . . . . . . . 45

5.3 Branch-and-Bound method . . . . . . . . . . . . . . . . . 49

5.3.1 Construction of convex relaxation . . . . . . . . . . . 51

5.3 .2 Branching method ... . . . . . . . . . . 54

6 In Vivo Parameter Identification Method 57

6.1 Pre-processing clinical data . . . . . . . . . . . . . . . . . 59

6.2 Validation . . . . . . . . . . . . . . . . . . 61

6.2.1 Model parameters . . . . . . . . . . . . . . . . . 63

6.2 .2 Stress state . . . . . . . . . . . . . . . . . . . 64

6.3 Global optimization . . . . . . . . . . . . . . . . . . . 65

6.4 Extension to account for smooth muscle activity . . . . . . . . . 66

$\begin{array}{lll}7 & \text { Discussion and Conclusion } & 69\end{array}$

8 Outlook $\quad 71$

9 Review of Appended Papers $\quad 73$

$\begin{array}{ll}\text { Part II - Appended Papers } & 87\end{array}$

Paper I: An in vivo parameter identification method for arteries: numerical validation for the human abdominal aorta . . . . . . . . . . . 91

Paper II: Assessing the accuracy of the stress predicted by an in vivo parameter identification method for arteries . . . . . . . . . . 123

Paper III: Identification of mechanical properties of arteries with certification of global optimality . . . . . . . . . . . . . . 147 
Paper IV: In vivo parameter identification in arteries considering multiple levels of smooth muscle activity . . . . . . . . . . . . . 177 



\section{Abbreviations and Glossary}

AA abdominal aorta

B\&B Branch-and-Bound

CaM calmodulin

FE finite element

HGO Holzapfel-Gasser-Ogden (strain-energy function (Holzapfel et al., 2000))

IMT intima-media thickness

in silico Latin for within the computer, used in the context of examining something modeled on the computer

in situ Latin for in its original place, used in the context of examining something outside the living organism but under natural conditions

in vitro Latin for within the glass, used in the context of examining something outside the living organism

in vivo Latin for within the living, used in the context of examining something within the living organism

IPOPT Interior Point Optimizer (Wächter and Biegler, 2006)

KKT Karush-Kuhn-Tucker (conditions)

MAP mean arterial pressure

MLCK myosin light-chain kinase

MLCP myosin light-chain phosphatase

PI parameter identification

PWV pulse wave velocity

RMSE root-mean-square error 



\section{Part I}

\section{Summary of the Work}





\section{Introduction}

The leading cause of death in the western world are cardiovascular diseases. According to Wilkins et al. (2017) and Mozaffarian et al. (2016) cardiovascular diseases account for $37 \%$ and $31 \%$ of all deaths in the European Union and the United States of America, respectively. People at risk of developing cardiovascular diseases are generally found by screening for biomarkers, e.g. age, sex, and blood pressure (Curry et al., 2018, Vlachopoulos et al., 2015). These biomarkers are combined into one risk score which is used to predict the risk of developing cardiovascular diseases in the near future. Risk scores such as SCORE (Conroy et al., 2003) or the Framingham Risk Score (D'Agostino et al., 2008), which are used in the European Union and the United States of America respectively, are not flawless, however, and there is a constant search for better ways to assess the risk for cardiovascular disease development. One biomarker, which has attracted a lot of attention, is arterial stiffness (Laurent et al., 2012, Van Sloten et al., 2014).

Arterial stiffness reflects the mechanical properties of the underlying tissue which is determined by its constituents and their arrangement. From a mechanical perspective the most important constituents are smooth muscle cells and the connective fibers elastin and collagen. While elastin and collagen build up an extracellular network providing passive resilience to the arterial wall, smooth muscle cells modulate arterial stiffness through their ability to actively contract or relax. It is well accepted that the development of cardiovascular diseases is associated with changes of these constituents, in particular elastin and collagen (Burton, 1954, Cocciolone et al., 2018, Ecobici and Stoicescu, 2017, Laurent et al., 2005, 2006, Roy, 1881, Tsamis et al., 2013, Vorp, 2007). In the case of abdominal aortic aneurysms it has been suggested that the loss of elastin initiates dilation and the aorta attempts to compensate by an increased collagen synthesis (Choke et al., 2005). An increased collagen synthesis, in general, stiffens the arterial wall which is associated with systemic hypertension (Laurent et al., 2012, O'Rourke, 1990, Zieman et al., 2005). A change in arterial stiffness appears therefore to precede cardiovascular diseases and the possibility to identify the mechanical properties of arteries inside the human body has, therefore, the potential to greatly facilitate disease diagnostization, treatment, and monitoring.

The potential of quantifying arterial stiffness has been recognized by the medical community for a long time and different measures have been introduced to clinically assess patients. Measures such as the pulse wave velocity (PWV) (Bramwell and Hill, 1922), the pressure-strain elastic modulus (Ep) (Peterson et al., 1960), and the stiffness index $(\beta)$ (Kawasaki et al., 1987) are commonly used within the 
clinic to quantify arterial stiffness (Ecobici and Stoicescu, 2017, Laurent et al., 2006, Mancia et al., 2007). These measures reflect the overall arterial stiffness and, typically, rely on a linearized response. The arterial wall exhibits distinctive non-linear stiffening (Roach and Burton, 1957), however, and these arterial stiffness measures are, therefore, blood pressure dependent (Zieff et al., 2019). Another limiting assumption is that these measures do not distinguish between the stiffness provided by the intricate fiber network and the active stiffness modulation by smooth muscle cell contraction.

To address these shortcomings, several research groups have proposed methods that use non-linear continuum-mechanical models whose parameters are related to the stiffness of the arterial wall constituents (Masson et al., 2008, Schulze-Bauer and Holzapfel, 2003, Smoljkić et al., 2015, Spronck et al., 2015, Stålhand, 2009, Wittek et al., 2016). The model parameters are identified by fitting the model's response to clinical measurements.

Data that can be measured inside the human body, i.e. in vivo, is typically limited to time-resolved blood pressure, inner radius of the (assumed) circular artery and in some cases thickness of the arterial wall. Information about the loading in the longitudinal direction and constraints posed by surrounding tissue, organs, and bones is not available. Furthermore, the level of smooth muscle activation, degree of residual stress, and the stress-free state of an artery are unknown.

This limited amount of in vivo measurable data restricts the number of model parameters which can be uniquely identified (Reesink and Spronck, 2019, Stålhand et al., 2004). Introducing too many model parameters leads to overparameterization, meaning that not one set of parameters describes the unique mechanical properties of the artery but that there exist many different model parameter combinations which describe the properties equally well. In the context of using the identified parameters as surrogates for arterial stiffness it becomes even more apparent that an overparameterized model must be avoided, because which parameter combination should clinicians use to diagnose diseases or as intervention criterion?

Overparameterization can be prevented by adding measurements exciting other modes of the arterial model, which is unlikely to be feasible in vivo, by fixing some model parameters to values reported in the literature, or by reducing the complexity of the model. However, if many model parameters are fixed to reported values the question arises how representative these parameters are for the individual patient? Similarly, how well does a simplified arterial model represent the mechanical properties of an artery? These are typical questions to be answered by a proper validation. Out of the cited methods only the one proposed in Smoljkić et al. (2015) has been (numerically) validated, however, only using one artery.

Another issue that arises when fitting a model's response to clinical data is the possibility to identify a suboptimal combination of model parameters. Fitting is an iterative process during which the difference between the model's response and the measured data is minimized until no progress can be achieved anymore. This process is performed by solving a minimization problem; typically the weighted leastsquares differences of measured and predicted pressure-radius response (Masson et al., 2008, Schulze-Bauer and Holzapfel, 2003, Smoljkić et al., 2015, Spronck et al., 
2015, Stålhand, 2009, Wittek et al., 2016). The non-linear continuum-mechanical model makes the minimization problem non-convex, however, and such problems typically posses suboptimal (local) solutions that are not the desired best-fit (global) solution (Nocedal and Wright, 1999). This issue is typically addressed by using a gradient-based optimization algorithm, which identifies a local solution, initiate this algorithm from multiple starting points, and take the best local solution as the global solution (Smoljkić et al., 2015, Spronck et al., 2015, Wittek et al., 2016). Unfortunately, there is no guarantee that this heuristic method finds the global solution.

\subsection{Aim of the work}

The aim of this Ph.D. project is to propose an in vivo parameter identification method, which can determine the mechanical properties of arteries within the human body, and address the shortcomings presented in the Introduction. In particular, the following four research questions are answered:

RQ1: How well does the proposed in vivo parameter identification method identify the mechanical properties of an artery?

RQ2: How well does the arterial model used in the in vivo parameter identification method in combination with the identified model parameters represent the examined artery?

RQ3: Is it feasible to employ deterministic global optimization methods to solve the minimization problem within the in vivo parameter identification method to obtain the global solution?

RQ4: How can the arterial model be extended to account for smooth muscle activity without causing overparameterization?

These four research questions are answered with respect to the proposed in vivo parameter identification method. The methodology used to answer these questions is, however, general and can be applied to other methods as well.

\subsection{Scope of the work}

As stated in Section 1.1 a method is proposed in this dissertation which is capable to determine the mechanical properties of arteries and the identified mechanical properties are supposed to facilitate disease diagnostization, treatment, and monitoring. This process is divided into three parts, see Figure 1:

Clinic: Measurements are performed in the clinic.

In vivo parameter identification method: The clinical data are pre-processed and afterwards used to identify the mechanical properties of an artery. 


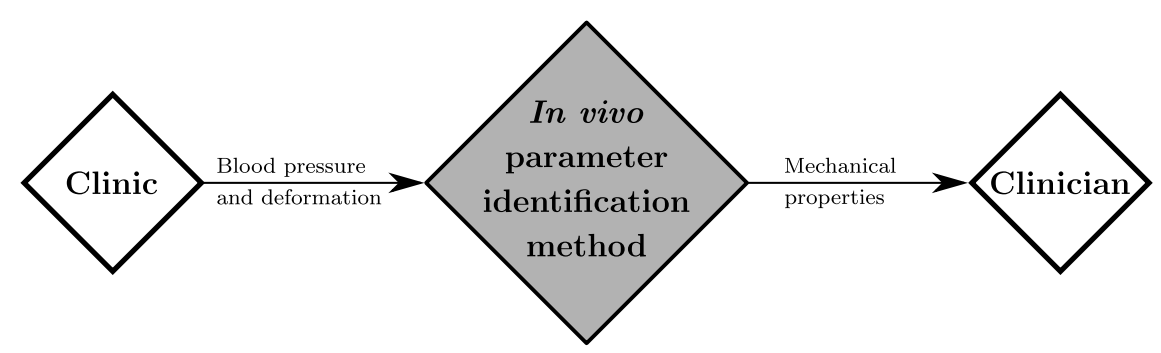

Figure 1: Overall project divided into parts.

Clinician: Use the identified mechanical properties to diagnose, treat, and monitor cardiovascular diseases.

To limit the scope of the work, only the in vivo parameter identification method is considered in this dissertation. How the clinical data are measured and how the identified mechanical properties can be used to guide clinicians exceed the scope of this work.

\subsection{Outline}

This dissertation builds upon the licentiate thesis Mechanical Properties of Arteries Identification and Application (Gade, 2019) presented in September 2019. Additional research has been conducted in the meanwhile and this dissertation represents the whole research outcome of this Ph.D. project. Some parts of the licentiate thesis are reused but not indicated as such to facilitate the flow of reading. The dissertation is structured as a compilation thesis and consists of two parts:

Part I - Summary of the Work, and

Part II - Appended Papers.

Part I is an extended summary integrating the research expressed in the appended papers. For that purpose, Part I begins with an introduction into the research area and highlights topics which have not been addressed sufficiently in the literature. These research gaps lead to four research questions addressed in this dissertation. In order to provide the necessary background for this dissertation, the physiology of arteries with a focus on their mechanical properties and the fundamentals of continuum mechanics are introduced. The mechanically relevant characteristics of an artery are translated into a general continuum-mechanical model which is the basis for a simplified arterial model used in the in vivo parameter identification method. Afterwards, it is explained how parameters can be identified using in vivo data in general. Next, the proposed in vivo parameter identification method is presented. Assumptions and limitations are scrutinized within the respective sections and the separate concise discussion is integrated into the conclusion in 
which the research questions are answered. Finally, an outlook is given and the appended papers are reviewed.

Part II contains the four academic papers produced within the Ph.D. project: Paper I has been peer-reviewed and published in an international journal. Papers II, III, and IV have been submitted to international journals and are currently undergoing peer-review. Papers I, II, III, and IV are connected to the research questions $R Q 1-4$, respectively. 



\section{Arteries}

This chapter provides a brief overview about the systemic arterial system. The focus is on the main characteristics of arterial tissue in general and of the human abdominal aorta in particular.

\subsection{Function and structure}

The primary function of systemic arteries in the cardiovascular system is to provide a conduit system for transportation of blood from the heart to the capillaries, where oxygen and nutrients are exchanged for carbon dioxide and other waste products of the cells inside the body.

Arteries are structurally similar throughout the body, but differ in detail, depending on their specific function in the cardiovascular system. Arteries near the heart possess large lumen diameters and are elastic. With increasing distance from the heart the arterial size decreases gradually and they contain more smooth muscle cells. Arteries are, therefore, typically categorized as being of elastic or muscular type and prominent members are the aorta and the femoral artery, respectively (Humphrey, 2002, Tortora and Derrickson, 2012).

Due to their compliant behavior, elastic arteries distend greatly when the heart contracts (systole) and blood is ejected from the left ventricle into the aorta. This dilation stores parts of the energy and by recoiling of the arterial wall when the heart muscle relaxes (diastole) the blood flow is further propelled. This, so called, Windkessel effect dampens the pressure difference and provides the capillaries with an almost constant blood pressure throughout the cardiac cycle (Frank, 1990).

The role of muscular arteries in the cardiovascular system is to regulate blood flow by adjusting the activation level of the contractile smooth muscle cells and the accompanied change in the lumen area. An example of this adjustment is that during physical exercise, blood flow into the exercising limbs is promoted while it is reduced in other parts of the body (Atkinson et al., 2015, Green et al., 2017).

Independent of arterial type, the arterial wall consists of three layers (tunics), see Figure 2: the intima (tunica interna); the media (tunica media); and the adventitia (tunica externa). These layers are separated by elastic laminae: the internal elastic lamina between the intima and the media; and the external elastic lamina between the media and the adventitia.

The intima is the innermost layer of the arterial wall and has direct contact with the blood flowing through the lumen, see Figure 2. At its interface, a thin layer 


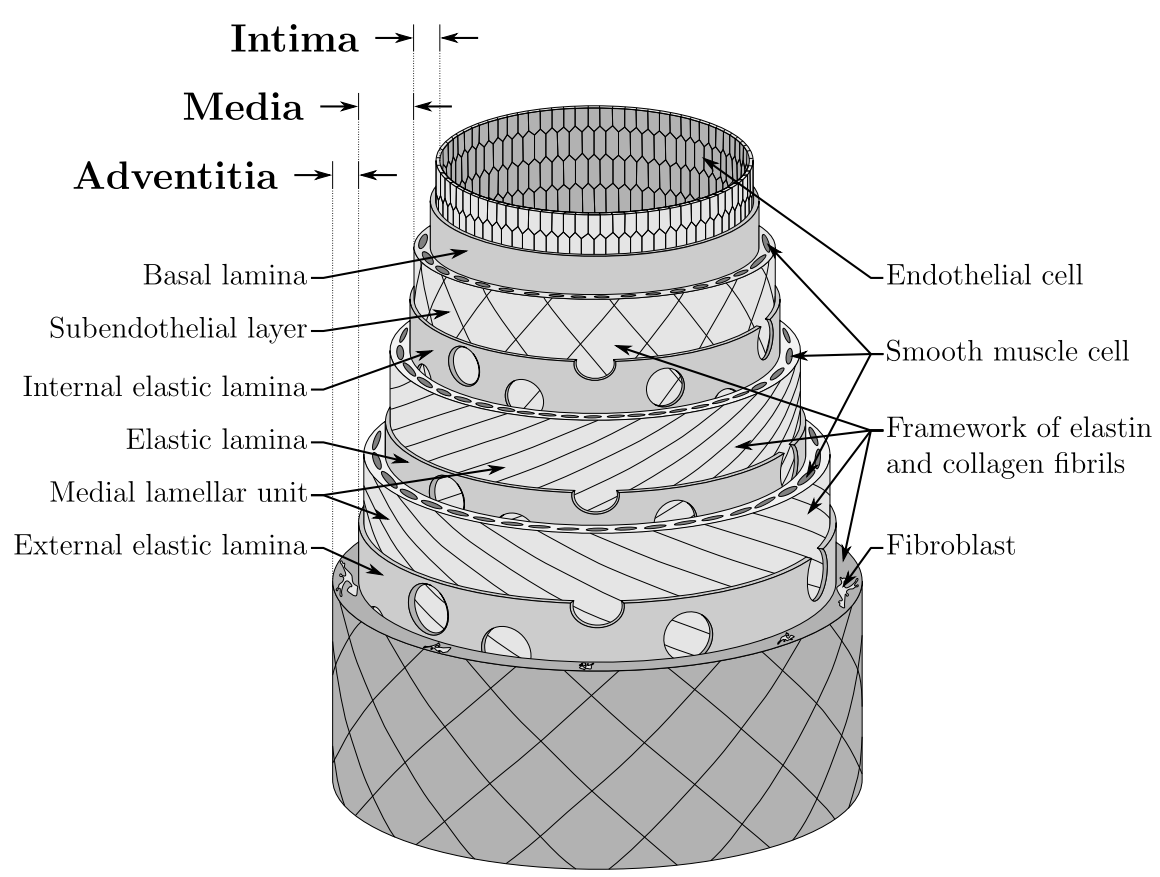

Figure 2: Schematic drawing of a healthy elastic artery.

of flattened cells, the endothelium, is located which act as a barrier to control the diffusion of blood constituents into the arterial wall and vice versa. Furthermore, the endothelium is biologically active and synthesizes for example growth and smooth muscle regulating substances (Humphrey, 2002, Tortora and Derrickson, 2012). The endothelium is held in place by the basal lamina (basement membrane) consisting of collagen fibers. In elastic arteries, such as the aorta, the basal lamina is followed by a subendothelial layer composed of connective tissue and smooth muscle cells (Humphrey, 2002, Rhodin, 2014). The intima is very thin compared to the other two tunics and, therefore, of minor mechanical relevance. In certain pathologies however, e.g. atherosclerosis, the intima thickens and stiffens substantially and may become mechanically relevant (Holzapfel et al., 2000).

The middle layer of the artery, the media, is the thickest of the three layers and consists primarily of smooth muscle cells held together by a framework of elastin and collagen fibrils embedded in an aqueous ground substance (Humphrey, 2002, Rhodin, 2014). In elastic arteries, the smooth muscles cells are arranged in medial lamellar units which are concentric layers separated by fenestrated elastic laminae, see Figure 2. Up to 60 of these structured subunits are found in the aorta (Rhodin, 2014). The elastic laminae, and therefore the medial lamellar units, are less well-defined in muscular arteries and there the media appears as one thick layer (Humphrey, 2002, Rhodin, 2014). 
The adventitia is the outermost layer of a blood vessel and believed to serve primarily as a protective sheath against over-distension (Holzapfel et al., 2000, Humphrey, 2002, Schulze-Bauer et al., 2002). For this purpose it consists of elastin and wavy collagen fibers which give the blood vessel high resilience. Besides these two constituents, various cells, e.g. fibroblasts, and in large elastic arteries vasa vasorum, which are small vessels to supply the vascular wall with blood, are present (Humphrey, 2002, Nichols and O'Rourke, 2005, Rhodin, 2014). The thickness of the adventitia depends strongly on arterial type and location. While some arteries do not have an adventitia, e.g. cerebral blood vessels, the adventitia comprises approximately $30 \%$ of the wall thickness in the elastic abdominal aorta (Holzapfel et al., 2007) and $25 \%$ in the muscular femoral artery (Auer et al., 2010).

\subsection{Mechanical properties}

A typical response of an artery is shown in Figure 3. During systole, the pressure inside the vessel rises quickly caused by the contraction of the heart, see Figure 3a. The deformation response of the artery, displayed in terms of inner radius in Figure $3 \mathrm{~b}$, is almost linear in the beginning but the vascular wall stiffens exponentially at higher pressures, cf. $14 \mathrm{kPa}$ in Figure $3 \mathrm{~b}$. This characteristic behavior is primarily associated with the network of elastin and collagen fibers inside the vascular wall. While elastin fibers are already stretched in the low pressure regime and limit the deformation, the wavy collagen fibers get successively straightened as the blood pressure increases. The continuous recruitment of collagen fibers gives rise to the exponential stiffening behavior seen in Figure 3b.

Both elastin and collagen fibers are primarily oriented in the circumferential-

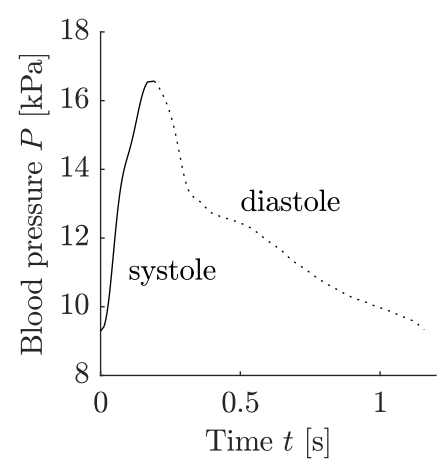

(a) Pressure-time curve

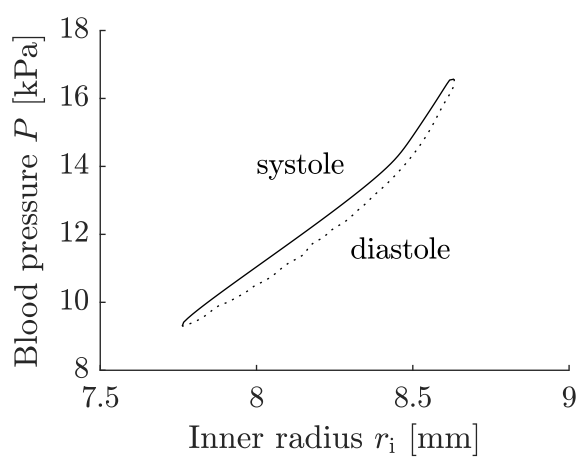

(b) Pressure-radius curve

Figure 3: A typical in vivo-recorded cardiac cycle of the abdominal aorta in a healthy 24 -year-old male. The solid and dotted lines denote the systolic and the diastolic phase, respectively. Data is taken from Sonesson et al. (1994). 
axial plane (Gundiah et al., 2007, Humphrey, 2002, Rhodin, 2014, Schriefl et al., 2012), cf. Figure 2. On a macroscopic level, elastin may be regarded as isotropic (Gundiah et al., 2007, 2009), while collagen is anisotropic due to its organized arrangement (Laksari et al., 2016, Nichols and O'Rourke, 2005, Schriefl et al., 2015). Two families of collagen fibers are found both in the media and the adventitia of an abdominal aorta, and they are symmetrically arranged around the circumferential direction. The preferred direction of collagen fibers in the media and the adventitia is more towards the circumferential and the axial direction, respectively (Schrief et al., 2012). The alignment of the collagen fibers causes the distinct anisotropic behavior of the vascular wall and couples the axial and circumferential direction.

The coupling of the circumferential and the axial direction is associated with an arterial characteristic which is beneficial in an energetical context. When an elastic artery is cut out of the body it generally retracts in the axial direction (Horný et al., 2011, Schulze-Bauer et al., 2003, Van Loon et al., 1977, Weizsäcker et al., 1983) (elongating elastic arteries during excision are reported in elderly men (Schulze-Bauer et al., 2003) and some muscular arteries do not change their length to facilitate wound healing (Tortora and Derrickson, 2012)). The arterial system can be described as a closed system of pipes subjected to varying pressure. Such a system does not only expand in the circumferential direction, see Figure 3b, but also axially. In quasi-static experiments of excised arterial rings, i.e. in vitro, it has been shown that if the tissue is stretched to the axial prestretch it had inside the body, i.e. in situ, the applied axial force necessary to hold the arterial ring in place is approximately constant in the physiological pressure range (Schulze-Bauer et al., 2003, Van Loon et al., 1977, Weizsäcker et al., 1983). This characteristic of an artery is energetically efficient, since no axial work is performed during the cardiac cycle, and is enabled by the fiber structure coupling the circumferential and axial direction, see Figure 4.

As pointed out in Section 2.1, the mechanical behavior of arteries is not solely

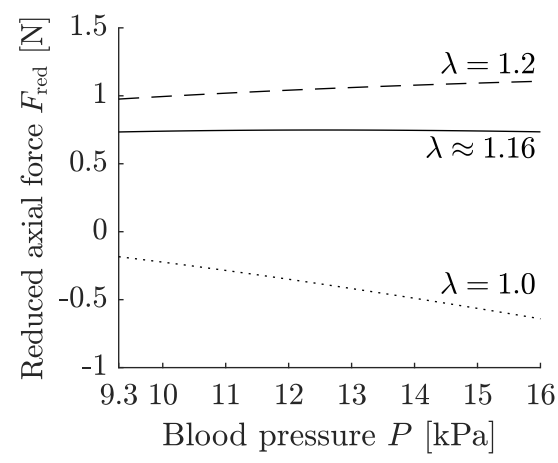

Figure 4: Reduced axial force throughout the cardiac cycle for three different axial prestretches $\lambda$ of an abdominal aorta. Data is taken from Paper I and corresponds to set 2 (F63). 
described by a passive response, but also affected by smooth muscle cells present in the vascular wall. Smooth muscle cells are primarily oriented around the circumferential direction, allowing them to actively constrict or dilate the lumen of the vessel when they contract or relax, respectively (Dobrin, 2011, Rhodin, 2014, Takamizawa et al., 1992). Smooth muscle cells contract slowly, in the order of ten seconds or more until peak tension (Dobrin, 2011, Somlyo and Somlyo, 1992), and thus do not change their contracted state during the cardiac cycle which is in the order of one second or less, cf. Figure 3a. Once contracted, smooth muscle cells maintain this state under minimal energy expenditure for long periods of time (Somlyo and Somlyo, 1992, Tortora and Derrickson, 2012). More information about smooth muscle contraction is found in Section 2.3.

Smooth muscle cells are also believed to cause viscoelastic effects in arteries, since these effects are increased in muscular arteries (Learoyd and Taylor, 1966) and can be reduced by inactivating smooth muscle cells (Cox, 1978a). The viscoelastic effects are expressed in several ways. For example, the load-deformation path during the cardiac cycle is different between the systolic and the diastolic phase, demonstrating hysteresis under cyclic loading, see Figure 3b. Other viscoelastic effects are stress relaxation under constant extension and creep under constant load (Fung et al., 1979).

Another mechanical characteristic of arterial tissue is that it approximately preserves its volume during deformation. The nearly incompressible behavior is attributed to the histology of the arterial wall, i.e. a structured composite of elastin, collagen, and smooth muscle cells in an aqueous ground substance (Humphrey, 2002, Rhodin, 2014). Several studies have tried to quantify the degree of compressibility, but it is experimentally difficult to accurately detect the small volume changes which arteries exhibit under physiological conditions. Recently it has been reported that in female porcine the relative volume change in the physiological pressure range is up to $4.5 \%$ and $2.5 \%$ in the femoral (muscular) and carotid (elastic) artery, respectively. Furthermore, larger arteries demonstrated less volume change compared to smaller arteries (Yosibash et al., 2014).

With respect to the loading situation it has already been pointed out, that an artery is prestretched in situ and subjected to blood pressure typically ranging from $9-16 \mathrm{kPa}$ (Sonesson et al., 1994), see Figure 3. The complete loading situation is, however, more complex since an artery is constrained by surrounding tissue, organs, and bones (Humphrey, 2002). To avoid accounting for these constraints explicitly (Kim et al., 2013), their effect on the artery is typically simplified to a pressure acting on the outside of the arterial wall (Humphrey and Na, 2002, Masson et al., 2008, Singh and Devi, 1990, Wittek et al., 2016). The magnitude of this perivascular pressure is reported to be between $0.67-0.93 \mathrm{kPa}$ in a normal population (De Keulenaer et al., 2009). The perivascular pressure decreases the transmural normal stress, which ranges from the blood pressure at the inside to the perivascular pressure at the outside, and, therefore, lowers the stress state that the arterial wall experiences, see Figure 5.

The flow of pressurized blood does not only cause a normal stress of $9-16 \mathrm{kPa}$ at the endothelium, but also induces a shear stress of approximately 1.5 Pa (Humphrey, 


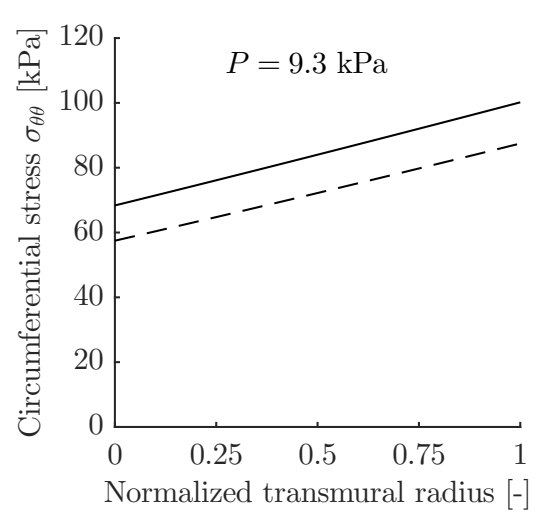

(a) Circumferential stress state

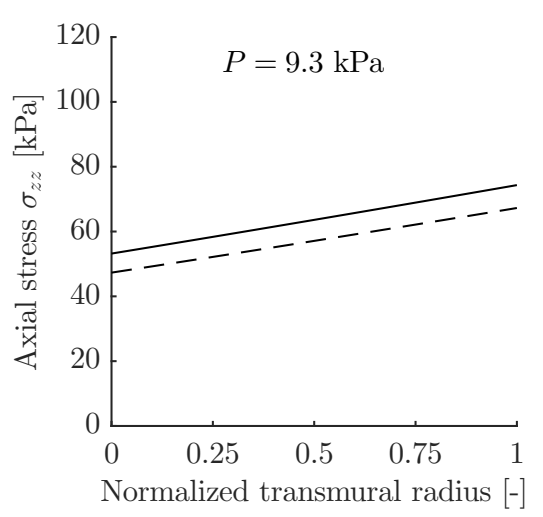

(b) Axial stress state

Figure 5: Transmural cirumferential and axial stress state at diastolic arterial pressure in an abdominal aorta. The solid and dashed lines represent the case without and with a constant perivascular pressure of $0.93 \mathrm{kPa}$, respectively. Data is taken from Paper I and corresponds to set 2 (F63).

2002). Although the shear stress is several orders of magnitude smaller than the normal stress, it has a profound effect on arterial wall mechanics since it is generally accepted that the arterial wall adapts in such a way to keep the shear stress at the homeostatic value of $1.5 \mathrm{~Pa}$ (Humphrey, 2002, Rodbard, 1975). If the shear stress is higher, e.g. due to increased blood flow, the endothelium releases factors relaxing smooth muscle cells to dilate the lumen and restore the shear stress to its homeostatic value. Correspondingly, if shear stress is lower, endothelial-mediated vasoconstriction occurs. With persisting deviation from this homeostatic shear stress value, the artery adapts in the long term its microstructure by growth and remodeling.

Another characteristic associated with growth and remodeling of the arterial wall is the existence of stress in an unloaded artery, i.e. residual stress. If an artery is cut radially it typically springs open to a horse-shoe like geometry, revealing a state of compression at the inner and tension at the outer boundary, see Figure 6 . The opening of the cut arterial segment is conveniently measured with the opening angle $\Phi_{0}$ (Chuong and Fung, 1986). Arteries do not necessarily open up into a cylindrical sector (Holzapfel et al., 2007, Labrosse et al., 2013) and there is no consensus in the literature whether the cut-open arterial segment is stress-free or not. Some studies show that one stress releasing cut is sufficient (Fung and Liu, 1989, Han and Fung, 1996), but there is more evidence that residual stress is more complex and cannot be described by a single opening angle (Greenwald et al., 1997, Taber and Humphrey, 2002, Vossoughi et al., 1993). Residual stress is probably even layer- and constituent-dependent (Holzapfel et al., 2007, Matsumoto et al., 2004, Saini et al., 1995, Schulze-Bauer et al., 2003, Zeller and Skalak, 1998). Nevertheless, 


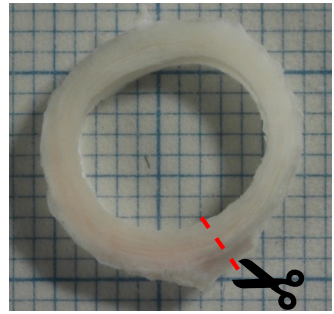

(a) Uncut

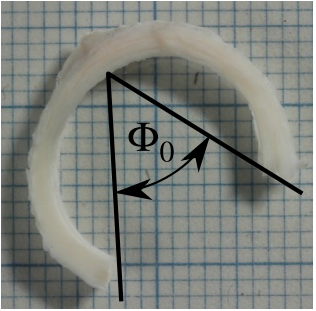

(b) Cut-open

Figure 6: Porcine arterial segment before and after a radial cut (courtesy of Jerker Karlsson).

the residual stress is commonly measured in terms of the opening angle due to its simplicity.

Residual stress has far reaching consequences on the overall stress state inside the arterial wall. If no residual stress would be present, the inner side of the wall would be subjected to much higher stretch and stress compared to the outer part, see Figure 7. The incorporation of residual stress lowers transmural gradients. In

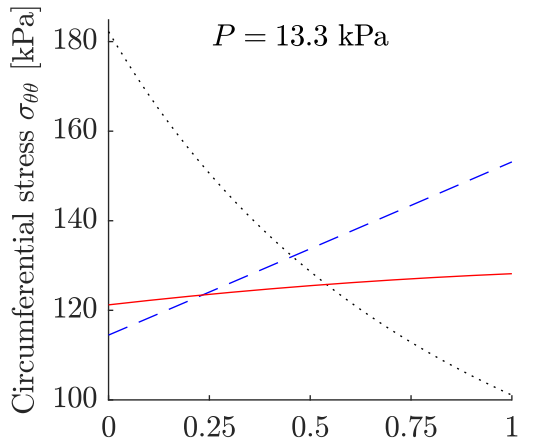

Normalized transmural radius [-]

(a) Stress

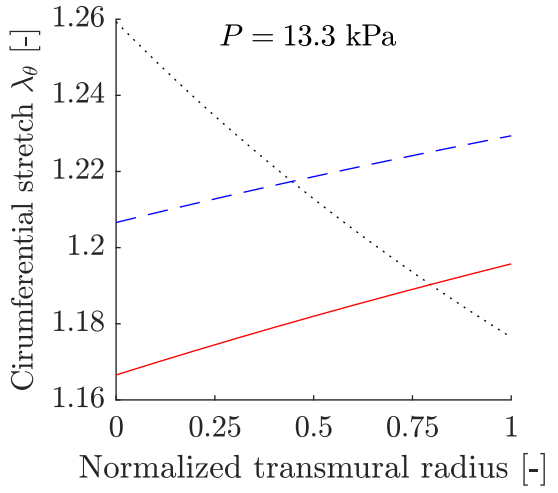

(b) Stretch

Figure 7: Transmural cirumferential stress and stretch state at mean arterial pressure in an abdominal aorta. The dotted black lines represent the case without residual stress, the dashed blue lines once residual stress is introduced, and the solid red lines denote the case when residual stress and smooth muscle activity are accounted for. Data is taken from Paper I and corresponds to set 2 (F63). Smooth muscle activity is introduced using the model proposed in Rachev and Hayashi (1999) with $S=40 \mathrm{kPa}$ and it is assumed that there is a gradual decrease of the number of smooth muscle cells from the inside to the outside of the artery. 
this context the uniform strain hypothesis (Takamizawa and Hayashi, 1987) and the uniform stress hypothesis (Fung, 1991) have been proposed. They suggest that the artery grows and remodels itself such that the circumferential stretch and stress are transmurally uniform at mean arterial pressure (MAP), respectively. As can be seen in Figure 7 the introduction of residual stress does not remove transmural gradients completely and it has been suggested that the combination of residual stress together with smooth muscle activity homogenizes transmural gradients (Humphrey and Wilson, 2003, Rachev and Hayashi, 1999).

\subsection{Smooth muscle cell contraction}

In order to understand how smooth muscle cells contract their microscopic structure needs to be introduced. Smooth muscle cells are spindle-shaped with a length of $30-200 \mu \mathrm{m}$ and a diameter of $3-8 \mu \mathrm{m}$, see Figure 8 (Tortora and Derrickson, 2012). Structural integrity is provided by their cytoskeleton which consists of, so called, dense bodies connected by (intermediate) filaments. The dense bodies also act as anchoring points for thin filaments which consist mostly of the protein actin. The actin filaments are interdigitating with the thick filaments containing mostly myosin and together they act as the contractile unit of the smooth muscle cell. It is the relative sliding of these two filaments which pulls the dense bodies towards each other causing the contraction of the smooth muscle cell, see Figure 8 (b) (Huxley and Niedergerke, 1954, Huxley and Hanson, 1954).

The relative sliding is initiated by the autonomic nervous system, stretching of the smooth muscle cell, hormones, or other chemical substances. All of these cause calcium ions $\mathrm{Ca}^{2+}$ to flow from the extracellular space and the cell internal

(a) Relaxed smooth muscle cell

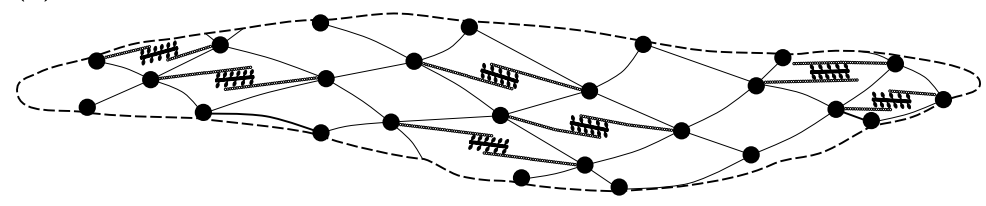

(b) Contracted smooth muscle cell

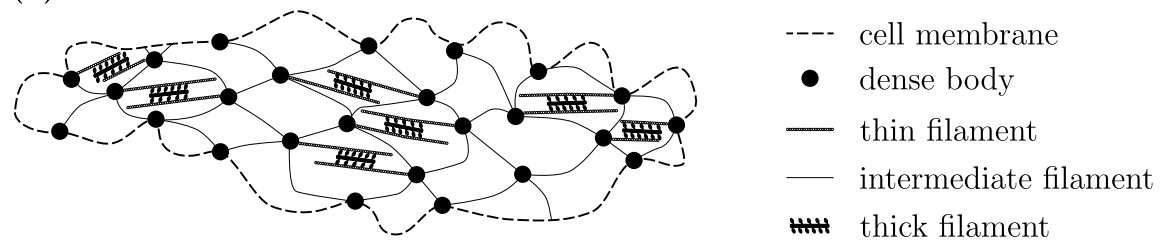

Figure 8: Structure of a vascular smooth muscle cell in its relaxed (a) and contracted (b) state. Figure is inspired by Murtada et al. (2012), Tortora and Derrickson (2012). 


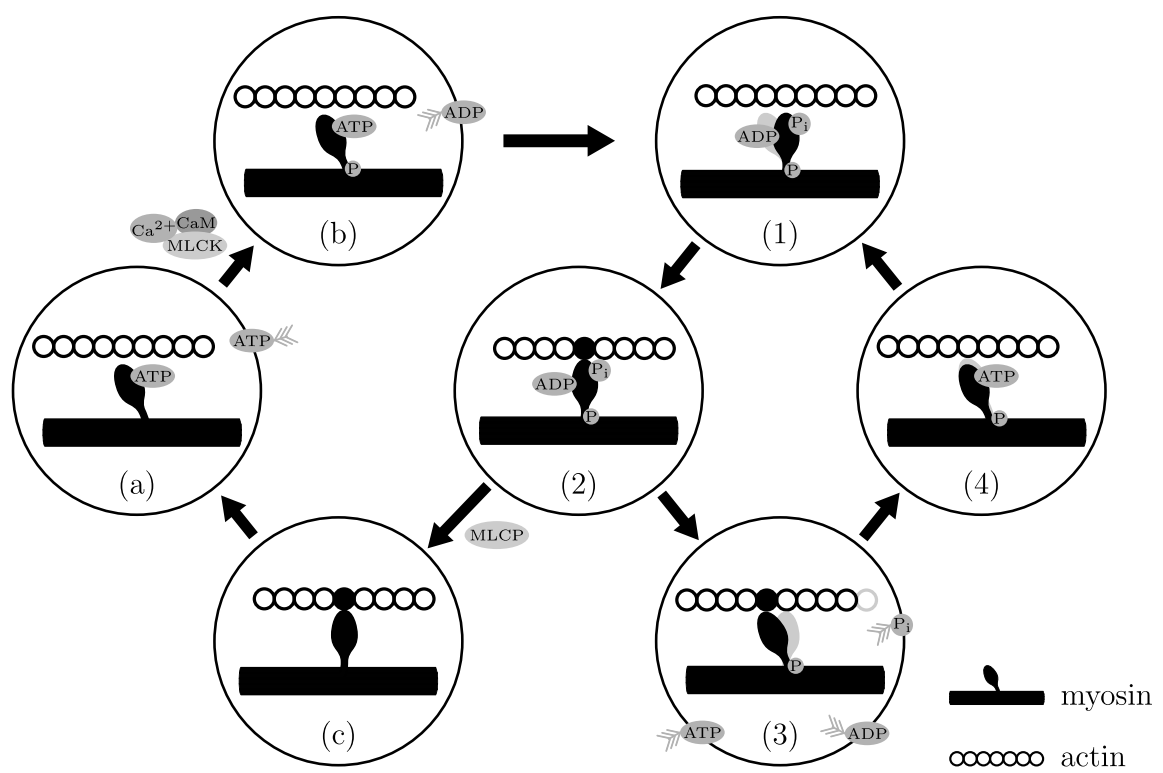

Figure 9: Schematic process of filament sliding. The structure is based on the model proposed in Hai and Murphy (1988) and the drawing is inspired by Murtada et al. (2010). The phospharylated regulatory light-chain in the neck region of the myosin is denoted by $\mathrm{P}$, while the inorganic phosphate triggering the power stroke is denoted by $\mathrm{P}_{\mathrm{i}}$ at the myosin head. A formed cross-bridge between myosin and actin is represented by a filled actin protein.

$\mathrm{Ca}^{2+}$-reservoir into the plasma of the cell, the cytoplasm. In the cytoplasm, $\mathrm{Ca}^{2+}$ binds to the regulatory protein calmodulin (CaM) which activates the enzyme myosin light-chain kinase (MLCK). The enzyme then phosphorylates the regulatory light-chain of the myosin by use of adenosin triphosphate (ATP), activating the myosin head, see Figure 9 (a) and (b). The activated myosin head now hydrolyzes a bound ATP into adenosin diphosphate (ADP) and one inorganic phosphate $\left(\mathrm{P}_{\mathrm{i}}\right)$, reorienting the head and energizing it, see Figure 9 (1). This is the onset of the, so called, cross-bridge cycle. The energized myosin head binds in step (2) to actin forming a, so called, cross-bridge. In the next step (3) the bound $\mathrm{P}_{\mathrm{i}}$ is released, triggering a power stroke which pulls the actin filament inwards. This is followed by the release of the attached ADP freeing up the corresponding binding site on the myosin head. Once a new ATP binds to the myosin head in step (4), the crossbridge detaches and the cross-bridge cycle repeats. The cross-bridge cycle stops once the enzyme myosin light-chain phosphatase (MLCP) removes the phosphor group from the regulatory light-chain of myosin inactivating its head. Dillon et al. (1981) postulated another state in which MLCP removes the phosphor group from the regulatory light-chain of myosin while the cross-bridge still persists, see Figure 9 (c). 


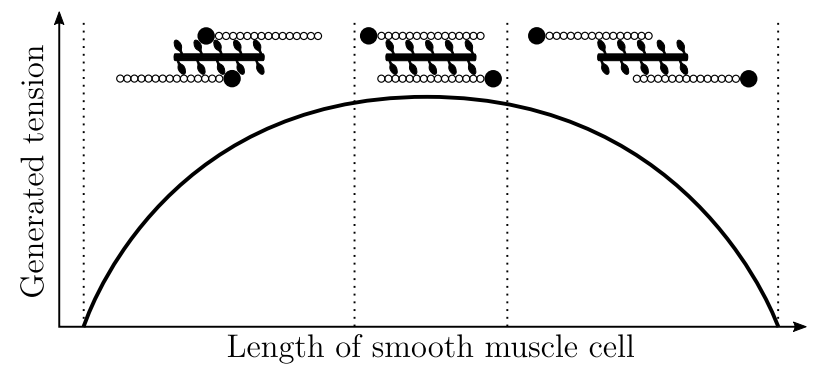

Figure 10: Schematic length-tension relationship of smooth muscle cells.

These, so called, latch-bridges cycle slowly (Murphy, 1982).

The described mechanism contracting a smooth muscle cell explains the characteristics observed during experiments. Once $\mathrm{Ca}^{2+}$ enters the cytoplasm it takes time until it binds to CaM which then activates MLCK which in turn phospharylates the myosin and the cross-bridge cycle is finally started (Arner, 1982, Tortora and Derrickson, 2012). Depending on the magnitude of the $\mathrm{Ca}^{2+}$ influx more myosin is phospharylated and more cross-bridges cycle simultaneously, resulting in a stronger contraction (Arner, 1982).

The relative position of myosin and actin also plays an important role (Cox, 1978b, Dobrin, 1973, Price et al., 1981). At some length of the smooth muscle cell, the thin and thick filaments are pulled apart and myosin cannot bind to actin anymore. Similarly, if cross-bridges have been cycling for some period of time myosin might have reached the dense body and cannot bind to actin either. In between these two extrema there is an optimal region where most cross-bridges are able to cycle and the muscle generates maximum tension. This length-tension relationship has a parabolic shape (Cox, 1978b, Dobrin, 1973, Price et al., 1981) and is expressed schematically in Figure 10.

The postulated slow cycling latch-bridges provide an explanation for why smooth muscle cells are able to maintain their contracted state under minimum energy expenditure (Murphy, 1982).

Finally, it has been observed that the generated tension of smooth muscle cells decreases as the shortening velocity increases (Arner, 1982). 


\section{Continuum Mechanics}

In the following, the concept of continuum mechanics is introduced to provide a basic understanding necessary to comprehend this dissertation. This chapter is supposed to be a basic introduction and the interested reader is referred to the original works by Holzapfel (2000), Humphrey (2002) which the following is based upon.

In continuum mechanics a body is approximated as a continuous aggregation of material with locally averaged properties. For every continuum-mechanical model the following five classes of relations need to be established:

1. Kinematics;

2. Definition of stress;

3. Balance principles;

4. Constitutive equation; and

5. Boundary conditions.

\subsection{Kinematics}

The kinematic relationship describes how a body moves and deforms in space through time. For this purpose, consider an arbitrary body at time $t=0$ and call the domain that this body occupies in three-dimensional Euclidean space the reference configuration $\mathscr{B}_{0}$, see Figure 11a. With advancing time, i.e. $t>0$, the body changes and now occupies another domain in space, denoted the current configuration $\mathscr{B}$. In order to describe the position of the body two Cartesian coordinate systems are introduced, see Figure 11a: one with base vectors $\mathbf{E}_{1}, \mathbf{E}_{2}$ and $\mathbf{E}_{3}$ for the reference configuration; and another one with base vectors $\mathbf{e}_{1}, \mathbf{e}_{2}$ and $\mathbf{e}_{3}$ for the current configuration. For every point $\mathbf{x} \in \mathscr{B}$ and $\mathbf{X} \in \mathscr{B}_{0}$, there exists a one-to-one correspondence expressed through the bijective function

$$
\mathbf{x}=\chi(\mathbf{X}, t)
$$

called the motion of the body. The gradient of this motion is called the deformation gradient defined as

$$
\mathbf{F}=\frac{\partial \chi(\mathbf{X}, t)}{\partial \mathbf{X}}
$$




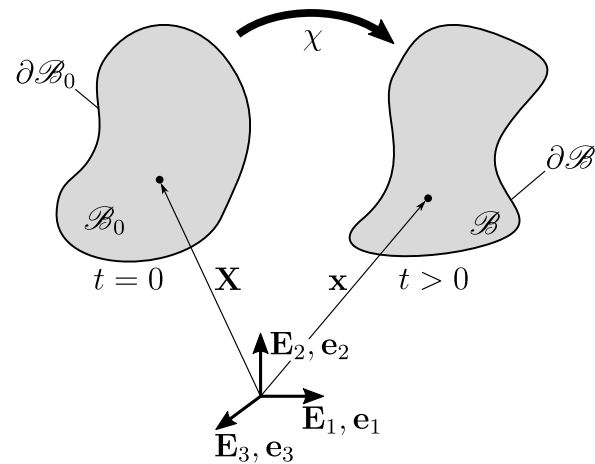

(a) Kinematics

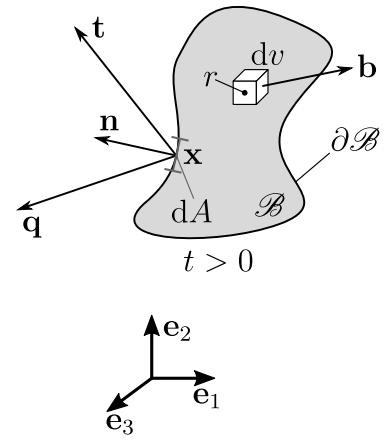

(b) Load state

Figure 11: Kinematics (a) and load state (b) of an arbitrary body.

The deformation gradient is the basis for all deformation measures in continuum mechanics and its determinant represents the change in volume between configurations. For example, a volume preserving deformation is given by

$$
\operatorname{det} \mathbf{F}=1
$$

where det denotes determinant. Furthermore, the deformation gradient can be decomposed using the polar decomposition theorem (Chadwick, 1999) as

$$
\mathbf{F}=\mathbf{R U},
$$

where $\mathbf{R}$ is a proper orthogonal second-order tensor representing a pure rigid-body rotation and $\mathbf{U}$ is a symmetric second-order tensor associated with a pure stretching of the body ${ }^{1}$. Hence, only the tensor $\mathbf{U}$ describes the deformation of a body and ideally a deformation measure should only consider this part. In this dissertation, the right Cauchy-Green stretch tensor $\mathbf{C}$ is used since it exhibits this beneficial characteristic:

$$
\mathbf{C}=\mathbf{F}^{\top} \mathbf{F}=\mathbf{U}^{\top} \mathbf{R}^{\top} \mathbf{R} \mathbf{U}=\mathbf{U}^{\top} \mathbf{I} \mathbf{U}=\mathbf{U}^{2},
$$

where $\mathbf{T}$ denotes the transpose of a tensor, $\mathbf{I}$ is the second-order identity tensor, and it has been used that the transpose of a proper-orthogonal tensor is equal to its inverse, i.e. $\mathbf{R}^{\mathbf{T}}=\mathbf{R}^{-1}$ (Chadwick, 1999).

\subsection{Definition of stress}

In this dissertation, the stress definition by Cauchy is used. Consider therefore a differential force vector $\mathrm{d} \boldsymbol{f}$ acting on the differential surface $\mathrm{d} A$ with unit normal

\footnotetext{
${ }^{1}$ Observe that pure rigid-body translations are not included in the deformation gradient.
} 
vector $\mathbf{n}$ and define the traction vector

$$
\mathbf{t}=\frac{\mathrm{d} \boldsymbol{f}}{\mathrm{d} A} .
$$

Cauchy's stress theorem then states that there exists a unique second-order tensor field called the Cauchy stress tensor $\boldsymbol{\sigma}(\mathbf{x}, t)$ which is independent of $\mathbf{n}$ such that $\mathbf{t}$ is a linear function of $\mathbf{n}$, i.e.

$$
\mathbf{t}(\mathbf{x}, t, \mathbf{n})=\boldsymbol{\sigma}(\mathbf{x}, t) \mathbf{n}
$$

\subsection{Balance principles}

Every body has to obey the fundamental laws of physics, denoted the balance principles. These fundamental laws are: conservation of mass; conservation of linear momentum; conservation of angular momentum; conservation of energy; and the entropy inequality. In the following the balance principles are first introduced in global (integral) form, i.e. integrated over some region of the body which is taken to be the complete body. Afterwards the balance principles are reformulated such that they hold for every point of the body, i.e. in local (differential) form.

\subsubsection{Conservation of mass}

Conservation of mass requires that the total mass $m$ in a closed system, i.e. a system that only allows transfer of energy over its boundary, remains the same. Hence, the mass of a body treated as a closed system does not depend on time, i.e.

$$
\dot{m}=0,
$$

where the superimposed dot represents the time derivative. It is, therefore, irrelevant in which configuration the body's mass is determined:

$$
m=\int_{\mathscr{B}} \varrho(\mathbf{x}, t) \mathrm{d} v=\int_{\mathscr{B}_{0}} \varrho_{0}(\mathbf{X}) \mathrm{d} V
$$

where $\varrho(\mathbf{x}, t)$ and $\varrho_{0}(\mathbf{X})$ are the mass densities, and $\mathrm{d} v$ and $\mathrm{d} V$ are infinitesimal volume elements inside the domains $\mathscr{B}$ and $\mathscr{B}_{0}$, respectively. Applying Reynold's transport theorem (Tadmor et al., 2011) to Eq. (9) one obtains

$$
\dot{m}=\int_{\mathscr{B}} \dot{\varrho}(\mathbf{x}, t)+\varrho(\mathbf{x}, t) \operatorname{div} \mathbf{v} \mathrm{d} v=0,
$$

where div denotes the divergence operator and $\mathbf{v}=\dot{\mathbf{x}}$ is the velocity field of the body. Equation (10) holds for any subbody of $\mathscr{B}$ and using the localization theorem (Gurtin, 1981), one obtains the local form

$$
\dot{\varrho}(\mathbf{x}, t)+\varrho(\mathbf{x}, t) \operatorname{div} \mathbf{v}=0 .
$$


In an open system, mass is allowed to enter through the system boundary. In this case conservation of mass requires, that the change of mass inside the system is equal to the inflow of mass.

All bodies will be treated as closed systems in this dissertation and open systems are, therefore, not considered anymore. Furthermore, bodies are considered to be homogeneous and field quantities such as the density are no longer functions of the position, i.e. $\varrho(\mathbf{x}, t)=\varrho(t)$. Moving forward the dependency on time $t$ is omitted.

\subsubsection{Conservation of linear momentum}

Conservation of linear momentum states that the rate of change of linear momentum, roughly mass times velocity, of a body is equal to all forces acting on the body. Consider therefore a body subjected to a traction force field $\mathbf{t}$ on its boundary $\partial \mathscr{B}$ and a body force $\mathbf{b}$, defined as force per unit mass, in its interior, see Figure 11b. Conservation of linear momentum requires (Holzapfel, 2000):

$$
\frac{\mathrm{d}}{\mathrm{d} t} \int_{\mathscr{B}} \varrho \mathbf{v} \mathrm{d} v=\int_{\partial \mathscr{B}} \mathbf{t} \mathrm{d} A+\int_{\mathscr{B}} \mathbf{b} \varrho \mathrm{d} v .
$$

Applying Cauchy's stress theorem in Eq. (7), Reynolds' transport theorem, conservation of mass, and the divergence theorem to convert a surface integral into a volume integral (Chadwick, 1999), Eq. (12) becomes

$$
\int_{\mathscr{B}}(\operatorname{div} \boldsymbol{\sigma}+\varrho \mathbf{b}-\varrho \dot{\mathbf{v}}) \mathrm{d} v=\mathbf{0}
$$

where $\mathbf{0}$ is the zero vector. Equation (13) holds for any subbody of $\mathscr{B}$ and using the localization theorem, one obtains the local form

$$
\operatorname{div} \boldsymbol{\sigma}+\varrho \mathbf{b}-\varrho \dot{\mathbf{v}}=\mathbf{0},
$$

which is known as Cauchy's first equation of motion. A special case of Cauchy's first equation of motion is the equilibrium equation in which $\dot{\mathbf{v}}=\mathbf{0}$.

\subsubsection{Conservation of angular momentum}

Conservation of angular momentum states that the rate of change of angular momentum, roughly angular velocity times moment of inertia, of a body is equal to all moments acting on the body. For a body subjected to a traction force field $\mathbf{t}$ on its boundary $\partial \mathscr{B}$ and a body force $\mathbf{b}$ in its interior, see Figure 11b, conservation of angular momentum requires (Holzapfel, 2000):

$$
\frac{\mathrm{d}}{\mathrm{d} t} \int_{\mathscr{B}} \mathbf{r} \times \varrho \mathbf{v} \mathrm{d} v=\int_{\partial \mathscr{B}} \mathbf{r} \times \mathbf{t} \mathrm{d} A+\int_{\mathscr{B}} \mathbf{r} \times \varrho \mathbf{b} \mathrm{d} v,
$$

where $\mathbf{r}=\mathbf{x}-\mathbf{x}_{\mathrm{a}}$ is the position vector to an arbitrary point $\mathbf{x}_{\mathrm{a}} \in \mathscr{B}$ and $\times$ denotes the cross product of two vectors. Identical to the conservation of linear momentum, 
Cauchy's stress theorem in Eq. (7), Reynold's transport theorem, conservation of mass, and the divergence theorem are applied to Eq. (15) to obtain (Holzapfel, 2000)

$$
\int_{\mathscr{B}} \mathbf{r} \times \varrho \dot{\mathbf{v}} \mathrm{d} v=\int_{\mathscr{B}} \mathbf{r} \times \operatorname{div} \boldsymbol{\sigma}+\mathcal{E}: \boldsymbol{\sigma}^{\top} \mathrm{d} v+\int_{\mathscr{B}} \mathbf{r} \times \varrho \mathbf{b} \mathrm{d} v,
$$

where : denotes the double contraction operator, $\mathcal{E}$ is the third-order permutation tensor defined as

$$
\mathcal{E}=\varepsilon_{i j k} \mathbf{e}_{i} \otimes \mathbf{e}_{j} \otimes \mathbf{e}_{k},
$$

where $\varepsilon_{i j k}=\left(\mathbf{e}_{i} \times \mathbf{e}_{j}\right) \cdot \mathbf{e}_{k}$ is the Levi-Civita symbol, $\cdot$ denotes the scalar product of two vectors, $\otimes$ denotes the dyadic (tensor) product, and $i, j, k=1,2,3$. Rearranging the terms in Eq. (16) and using the localization theorem, one obtains the local form

$$
\mathbf{r} \times(\varrho \dot{\mathbf{v}}-\operatorname{div} \boldsymbol{\sigma}-\varrho \mathbf{b})=\mathcal{E}: \boldsymbol{\sigma}^{\top}
$$

Here the left-hand side equals the zero vector due to Cauchy's first equation of motion in Eq. (14), thus implying that Cauchy's stress tensor must be symmetric, i.e.

$$
\boldsymbol{\sigma}=\boldsymbol{\sigma}^{\top}
$$

This equation is known as Cauchy's second equation of motion.

\subsubsection{Conservation of energy}

Conservation of energy states that the time rate of change of total energy in a closed system, which consists of mechanical, thermal, chemical etc. energy, equals the rate of energy entering the system. In this dissertation, only mechanical energy is considered, but the energy balance is formulated also accounting for thermal energy since it will make the entropy inequality more intuitive.

Considering a body subjected to a traction force field $\mathbf{t}$ on $\partial \mathscr{B}$, a body force $\mathbf{b}$ in $\mathscr{B}$, an inward heat flux $\mathbf{q} \cdot \mathbf{n}$ on $\partial \mathscr{B}$, and a heat source $r$ in $\mathscr{B}$, see Figure $11 b$, conservation of energy requires (Holzapfel, 2000):

$$
\frac{\mathrm{d}}{\mathrm{d} t} \int_{\mathscr{B}}\left(\frac{1}{2} \varrho \mathbf{v} \cdot \mathbf{v}+u\right) \mathrm{d} v=\int_{\partial \mathscr{B}}(\mathbf{t} \cdot \mathbf{v}-\mathbf{q} \cdot \mathbf{n}) \mathrm{d} A+\int_{\mathscr{B}}(\varrho \mathbf{b} \cdot \mathbf{v}+r) \mathrm{d} v
$$

where $u$ is the internal energy of the body defined per unit (current) volume. Observe in Eq. (20) that the heat flux $\mathbf{q} \cdot \mathbf{n}$ has a negative sign because $\mathbf{n}$ is the outward normal on $\partial \mathscr{B}$. Using Cauchy's stress theorem in Eq. (7), the divergence theorem, and conservation of mass, Eq. (20) can be rewritten as

$$
\frac{\mathrm{d}}{\mathrm{d} t} \int_{\mathscr{B}} u \mathrm{~d} v+\int_{\mathscr{B}} \varrho \dot{\mathbf{v}} \cdot \mathbf{v} \mathrm{d} v=\int_{\mathscr{B}}(\operatorname{div} \boldsymbol{\sigma} \cdot \mathbf{v}+\boldsymbol{\sigma}: \mathbf{d}-\operatorname{div} \mathbf{q}+\varrho \mathbf{b} \cdot \mathbf{v}+r) \mathrm{d} v,
$$


where $\mathbf{d}=\left(\mathbf{l}+\mathbf{l}^{\boldsymbol{\top}}\right) / 2$ is the rate of deformation tensor and $\mathbf{l}=\partial \mathbf{v} / \partial \mathbf{x}$ is the velocity gradient. Using further Cauchy's first equation of motion in Eq. (14), Reynold's transport theorem, and the localization theorem, Eq. (21) is presented in local form:

$$
\dot{u}+u \operatorname{div} \mathbf{v}=\boldsymbol{\sigma}: \mathbf{d}-\operatorname{div} \mathbf{q}+r .
$$

For future use, the Helmholtz free-energy $\Psi$, which measures the internal recoverable energy of a closed system, is introduced according to

$$
\Psi=U-T S,
$$

where $U$ is the internal energy of the body, $T$ is the absolute temperature, and $S$ is the entropy of the system. The quantities $\Psi, U$, and $S$ in Eq. (23) are defined per unit volume in the reference configuration. The internal energies in the current configuration $u$ and in the reference configuration $U$ are related by the change in volume between these two configurations, see Section 3.1:

$$
u=(\operatorname{det} \mathbf{F})^{-1} U \text {. }
$$

In case only mechanical energy is considered the Helmholtz free-energy coincides with the internal energy, i.e. $\Psi=U$.

\subsubsection{Entropy inequality}

The entropy inequality is the fundamental statement about the irreversibility of natural processes by stating that entropy can only be generated but never be destroyed. It is therefore postulated that the total production of entropy per unit time, $\Gamma(t)$, must be non-negative, i.e. (Holzapfel, 2000)

$$
\Gamma(t)=\frac{\mathrm{d}}{\mathrm{d} t} \int_{\mathscr{B}} s \mathrm{~d} v-\tilde{Q}(t) \geq 0,
$$

where $s=(\operatorname{det} \mathbf{F})^{-1} S$ is the entropy of the system defined per unit current volume and $\tilde{Q}(t)$ is the rate of entropy input. Typically it is assumed that the rate of entropy input is related to the heat flux $\mathbf{q} \cdot \mathbf{n}$ and the heat source $r$ by the relation (Holzapfel, 2000):

$$
\tilde{Q}(t)=\int_{\partial \mathscr{B}}-\frac{\mathbf{q}}{T} \cdot \mathbf{n} \mathrm{d} A+\int_{\mathscr{B}} \frac{r}{T} \mathrm{~d} v .
$$

Combining Eqs. (25) and (26) results in

$$
\Gamma(t)=\frac{\mathrm{d}}{\mathrm{d} t} \int_{\mathscr{B}} s \mathrm{~d} v+\int_{\partial \mathscr{B}} \frac{\mathbf{q}}{T} \cdot \mathbf{n} \mathrm{d} A-\int_{\mathscr{B}} \frac{r}{T} \mathrm{~d} v \geq 0,
$$

which is known as the Clausius-Duhem inequality. In order to present Eq. (27) in its local form, Reynold's transport theorem, the divergence theorem, and the localization theorem are applied to get

$$
\dot{s}+s \operatorname{div} \mathbf{v}+\operatorname{div} \frac{\mathbf{q}}{T}-\frac{r}{T} \geq 0 .
$$


By rewriting the term $\operatorname{div} \mathbf{q} T^{-1}$ as

$$
\operatorname{div} \frac{\mathbf{q}}{T}=\frac{1}{T} \operatorname{div} \mathbf{q}-\mathbf{q} \cdot \frac{\partial T^{-1}}{\partial \mathbf{x}}=\frac{1}{T} \operatorname{div} \mathbf{q}-\frac{\mathbf{q}}{T^{2}} \cdot \frac{\partial T}{\partial \mathbf{x}},
$$

and replacing the heat source term in Eq. (28) by Eq. (22) one obtains

$$
T(\dot{s}+s \operatorname{div} \mathbf{v})+-\frac{\mathbf{q}}{T} \cdot \frac{\partial T}{\partial \mathbf{x}}-\dot{u}-u \operatorname{div} \mathbf{v}+\boldsymbol{\sigma}: \mathbf{d} \geq 0 .
$$

Combining Eqs. (23), (24), and (30) one obtains an alternative local form of Eq. (28), namely:

$$
-\frac{\dot{\Psi}}{\operatorname{det} \mathbf{F}}-\dot{T} \mathbf{s}+\boldsymbol{\sigma}: \mathbf{d}-\frac{\mathbf{q}}{T} \cdot \frac{\partial T}{\partial \mathbf{x}} \geq 0 .
$$

In the case of an isothermal motion, i.e. $\dot{T}=0$, and no heat flux, i.e. $\mathbf{q}=\mathbf{0}$, Eq. (31) reduces to

$$
-\frac{\dot{\Psi}}{\operatorname{det} \mathbf{F}}+\boldsymbol{\sigma}: \mathbf{d} \geq 0
$$

which must hold for all motions of the body.

\subsection{Constitutive equation}

The constitutive equation represents the relation between the stress state and the motion of the body. In this dissertation, each body is treated as consisting of a perfectly elastic material, i.e. no entropy is produced by elastic deformation. Consider therefore the Helmholtz free-energy to be a function solely of a stretch tensor, i.e. $\Psi=\Psi(\mathbf{C})$. In this case the term $\dot{\Psi} / \operatorname{det} \mathbf{F}$ in Eq. (32) can be rewritten as

$$
\frac{\dot{\Psi}}{\operatorname{det} \mathbf{F}}=\frac{1}{\operatorname{det} \mathbf{F}} \frac{\partial \Psi}{\partial \mathbf{C}}: \dot{\mathbf{C}}=\frac{1}{\operatorname{det} \mathbf{F}} \frac{\partial \Psi}{\partial \mathbf{C}}:\left(2 \mathbf{F}^{\top} \mathbf{d} \mathbf{F}\right)=\frac{2}{\operatorname{det} \mathbf{F}} \mathbf{F} \frac{\partial \Psi}{\partial \mathbf{C}} \mathbf{F}^{\top}: \mathbf{d},
$$

where the relation $\dot{\mathbf{F}}=\mathbf{l} \mathbf{F}$ has been used. Substituting Eq. (33) in Eq. (32) shows that the following may be taken as a thermodynamically consistent constitutive equation:

$$
\boldsymbol{\sigma}=\frac{2}{\operatorname{det} \mathbf{F}} \mathbf{F} \frac{\partial \Psi}{\partial \mathbf{C}} \mathbf{F}^{\top}
$$

As can be seen in Eq. (34), the Cauchy stress tensor derives from the Helmholtz free-energy. A material that is represented by such a relationship is called a hyperelastic material. In this context, the Helmholtz free-energy $\Psi$ is commonly referred to as the strain-energy function.

In case the constitutive equation is supposed to describe an incompressible material, the deformation cannot be arbitrary but is constrained by $\operatorname{det} \mathbf{F}=1$, 
cf. Eq. (3), which is equivalent to the constraint $\operatorname{det} \mathbf{C}=1$. This constraint can be enforced directly through the strain-energy function by defining a constrained subclass of hyperelastic materials according to

$$
\Psi=\bar{\Psi}-\frac{p}{2}(\operatorname{det} \mathbf{C}-1),
$$

where $\bar{\Psi}$ is an unconstrained strain-energy function and $p$ is an arbitrary Lagrange multiplier enforcing the incompressibility constraint. Combining Eqs. (34) and (35) leads to the following constitutive equation for incompressible hyperelastic materials:

$$
\boldsymbol{\sigma}=-p \mathbf{I}+2 \mathbf{F} \frac{\partial \bar{\Psi}}{\partial \mathbf{C}} \mathbf{F}^{\top}
$$

The term $p \mathbf{I}$ is commonly called the reaction stress or the volumetric stress denoted by $\boldsymbol{\sigma}_{\mathrm{vol}}$. The second term in Eq. (36) is usually referred to as the isochoric stress $\overline{\boldsymbol{\sigma}}$.

Observe that $\Psi$ and $\bar{\Psi}$ are used interchangeably in this dissertation and the incompressibility constraint is introduced by stating the constitutive equation according to Eq. (36) directly.

\subsubsection{Constitutive equation in terms of invariants}

The strain-energy function $\Psi$ is a scalar-valued tensor function and should be invariant to superimposed rigid-body rotations (Holzapfel, 2000). The function can, therefore, be expressed in terms of the principal invariants of its argument instead (Gurtin, 1981). For an isotropic material, i.e. $\Psi=\Psi(\mathbf{C})$, it follows that $\Psi=\Psi\left(I_{1}(\mathbf{C}), I_{2}(\mathbf{C}), I_{3}(\mathbf{C})\right)$, where

$$
I_{1}(\mathbf{C})=\operatorname{tr} \mathbf{C}
$$

is the first invariant, tr denotes trace of a second-order tensor,

$$
I_{2}(\mathbf{C})=\frac{1}{2}\left[(\operatorname{tr} \mathbf{C})^{2}-\operatorname{tr} \mathbf{C}^{2}\right]
$$

is the second invariant, and

$$
I_{3}(\mathbf{C})=\operatorname{det} \mathbf{C}
$$

is the third invariant.

\subsubsection{Constitutive equation with directional properties}

Until now it was assumed that the strain-energy function $\Psi$ does not possess directional properties, i.e. that the material that $\Psi$ describes is isotropic. If the body is, however, reinforced with fibers, which possess different properties along the fibers compared to their transverse direction, the stress does not only depend on the deformation but also on the direction. 
Consider therefore a body which is reinforced with one family of uniformly distributed fibers oriented along the unit vector $\mathbf{M}$ defined in the reference configuration. The strain-energy function is taken to be an even function ${ }^{2}$ of $\mathbf{M}$ by letting the strain-energy function depend on the structural tensor $\mathbf{M} \otimes \mathbf{M}$, i.e. $\Psi=\Psi(\mathbf{C}, \mathbf{M} \otimes \mathbf{M})$ (Holzapfel, 2000).

By introducing, so called, pseudo-invariants, the strain-energy function can still be expressed in terms of invariants of its arguments. Besides the first three invariants in Section 3.4.1 describing the isotropic behavior of the matrix material, two new pseudo-invariants arise (Spencer, 1984):

$$
I_{4}(\mathbf{C}, \mathbf{M})=(\mathbf{M} \otimes \mathbf{M}): \mathbf{C}=\lambda_{\text {fib }}^{2},
$$

where $\lambda_{\text {fib }}$ is the fiber-stretch (Holzapfel, 2000); and

$$
I_{5}(\mathbf{C}, \mathbf{M})=(\mathbf{M} \otimes \mathbf{M}): \mathbf{C}^{2} .
$$

If the body is reinforced with a second family of equally distributed fibers oriented along the unit vector $\mathbf{N}$, the strain-energy function is given by $\Psi=$ $\Psi(\mathbf{C}, \mathbf{M} \otimes \mathbf{M}, \mathbf{N} \otimes \mathbf{N})$ and in addition to $I_{1}, \ldots, I_{5}$, four more pseudo-invariants arise (Spencer, 1984):

$$
\begin{gathered}
I_{6}(\mathbf{C}, \mathbf{N})=(\mathbf{N} \otimes \mathbf{N}): \mathbf{C} ; \quad I_{7}(\mathbf{C}, \mathbf{N})=(\mathbf{N} \otimes \mathbf{N}): \mathbf{C}^{2} \\
I_{8}(\mathbf{C}, \mathbf{M}, \mathbf{N})=\mathbf{M} \cdot \mathbf{C N} ; \quad \text { and } \quad I_{9}(\mathbf{M}, \mathbf{N})=(\mathbf{M} \cdot \mathbf{N})^{2}
\end{gathered}
$$

Here $I_{6}$ represents the squared stretch of the second set of fibers. The strain-energy function can analogously be extended with additional sets of fibers.

\subsection{Boundary conditions}

In order to solve a problem using a continuum-mechanical model, conditions at the boundary need to be specified ${ }^{3}$. In this dissertation, the traction vector $\mathbf{t}$ and displacement boundary conditions are specified along the boundary $\partial \mathscr{B}$.

\footnotetext{
${ }^{2}$ Here an even function means that the amount of recoverable energy does not depend on whether the fibers are in compression or tension.

${ }^{3}$ In case the problem is time-dependent also initial conditions need to be specified but such problems are not considered in this dissertation.
} 



\section{Mechanical Models for Arteries}

A mechanical model for arteries should ultimately reflect all mechanically relevant characteristics discussed in Chapter 2: large non-linear elastic deformations, anisotropy, passive and active behavior, (near) incompressibility, and residual stress. For simplicity viscoelasticity is neglected ${ }^{1}$ and only short time spans are considered, i.e. no arterial adaption in terms of growth and remodeling takes place. Furthermore, it is decided to base the arterial model on the concept of continuum mechanics such that the model describes the macroscopic behavior of arterial tissue.

Following the basics of continuum mechanics introduced in Chapter 3, a general continuum-mechanical model for arteries is established. In this context, relevant classes of relations are made explicit for the arterial model. The general model is the basis to create a simplified arterial model, the constitutive membrane model, which is used in the in vivo parameter identification method.

\subsection{General continuum-mechanical model for arteries}

\subsubsection{Kinematics}

The aorta is approximately cylindrical and tapers with distance from the heart (Humphrey, 2002). Assuming the aorta maintains this cylindrical shape, a small aortic segment is conveniently described as a thick-walled tube with cylindrical coordinates. Furthermore, the aorta is treated as a homogeneous single-layer structure, representing the combined response of the three layers. The stress-free state is taken to be the horse-shoe like geometry obtained after one radial cut, see Figure 6b. The residual stress is introduced into the arterial model by closing the cut-open geometry (Chuong and Fung, 1986), see Figure 12. Following Chuong and Fung (1986), three configurations are defined. The cut-open segment is assumed to be part of a rotationally symmetric domain $\mathscr{B}_{0}$ defined by

$$
\rho_{\mathrm{i}} \leq \rho \leq \rho_{\mathrm{o}}, \quad 0 \leq \phi \leq\left|2 \pi-2 \Phi_{0}\right|, \quad 0 \leq \xi \leq \zeta,
$$

where $\rho$ is the referential radius, the indices $i$ and o denote the inner and outer radius, $\Phi_{0} \neq \pi$ (corresponding to a straight line) is the opening angle, and $\zeta$ is the length of the arterial segment, respectively. In case of high opening angles, i.e. $\Phi_{0}>\pi$, the inner and outer radii become negative and exchange roles, demonstrating

\footnotetext{
${ }^{1} \mathrm{~A}$ simple extension of the arterial model to account for hysteresis under cyclic loading is to treat the systolic and diastolic phases as separate processes, cf. pseudo-elasticity (Fung, 1993).
} 


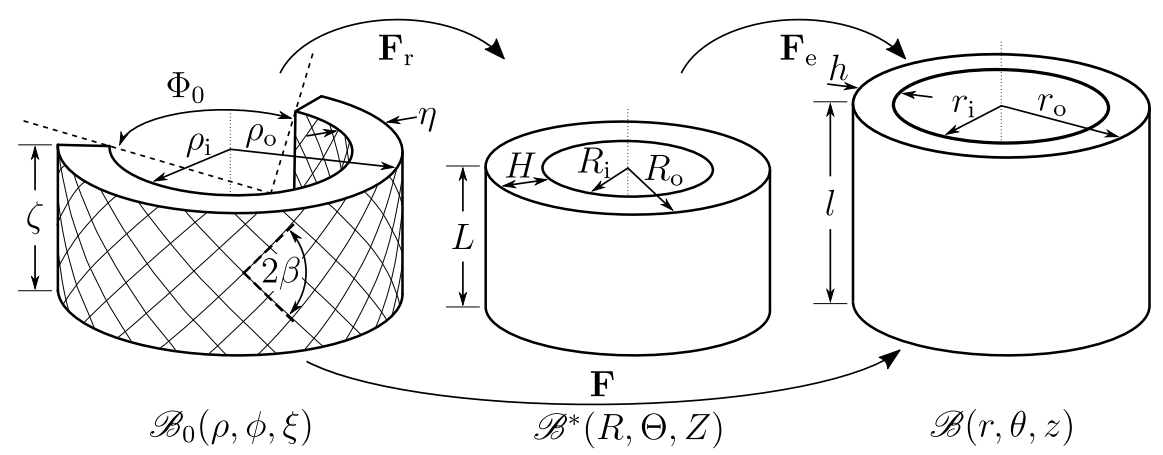

Figure 12: Stress-free $\left(\mathscr{B}_{0}\right)$, unloaded $\left(\mathscr{B}^{*}\right)$, and deformed $(\mathscr{B})$ configuration of an arterial segment. The coordinate triplets $(\rho, \phi, \xi),(R, \Theta, L)$, and $(r, \theta, l)$ are associated with the radial, circumferential, and axial direction of the respective domain (Paper I).

the change of curvature of the arterial segment (Labrosse et al., 2013). Closing the cut-open segment to form a perfect cylinder introduces residual stress and the unloaded configuration $\mathscr{B}^{*}$ is obtained, defined by

$$
R_{\mathrm{i}} \leq R \leq R_{\mathrm{o}}, \quad 0 \leq \Theta \leq 2 \pi, \quad 0 \leq Z \leq L,
$$

where $R$ is the unloaded radius and $L$ denotes the unloaded length. The final state is the deformed configuration $\mathscr{B}$ in which the artery is exposed to both an axial prestretch and a blood pressure. This configuration is defined by

$$
r_{\mathrm{i}} \leq r \leq r_{\mathrm{o}}, \quad 0 \leq \theta \leq 2 \pi, \quad 0 \leq z \leq l,
$$

where $r$ is the deformed radius and $l$ denotes the deformed length.

It is not essential to define the domain $\mathscr{B}^{*}$ since it is a special case of the deformed configuration. It is, however, illustrative to split the deformation into one part associated with residual stress and another part to the deformation caused by loading the artery. Accordingly, the deformation gradient $\mathbf{F}$ between $\mathscr{B}_{0}$ and $\mathscr{B}$ is split into

$$
\mathbf{F}=\mathbf{F}_{\mathrm{e}} \mathbf{F}_{\mathrm{r}}
$$

where $\mathbf{F}_{\mathrm{r}}$ describes the residual deformation between $\mathscr{B}_{0}$ and $\mathscr{B}^{*}$, and $\mathbf{F}_{\mathrm{e}}$ the deformation between $\mathscr{B}^{*}$ and $\mathscr{B}$. Cylindrical base vectors $\mathbf{E}_{\rho}(\phi), \mathbf{E}_{\phi}(\phi)$, and $\mathbf{E}_{\xi}$ are introduced for the reference configuration and $\mathbf{e}_{r}(\theta), \mathbf{e}_{\theta}(\theta)$, and $\mathbf{e}_{z}$ for both the unloaded and the deformed configuration. The base vectors are associated with the radial, circumferential, and axial direction, respectively. Note that the base vectors associated with the radial and circumferential direction depend on the circumferential position, as indicated, which is suppressed in the remainder of this dissertation. The deformation gradients read:

$$
\mathbf{F}_{\mathrm{r}}=\frac{\partial R}{\partial \rho} \mathbf{E}_{\rho} \otimes \mathbf{e}_{r}+k \frac{R}{\rho} \mathbf{E}_{\phi} \otimes \mathbf{e}_{\theta}+\frac{L}{\zeta} \mathbf{E}_{\xi} \otimes \mathbf{e}_{z},
$$




$$
\mathbf{F}_{\mathrm{e}}=\frac{\partial r}{\partial R} \mathbf{e}_{r} \otimes \mathbf{e}_{r}+\frac{r}{R} \mathbf{e}_{\theta} \otimes \mathbf{e}_{\theta}+\frac{l}{L} \mathbf{e}_{z} \otimes \mathbf{e}_{z}
$$

and

$$
\mathbf{F}=\frac{\partial r}{\partial \rho} \mathbf{E}_{\rho} \otimes \mathbf{e}_{r}+k \frac{r}{\rho} \mathbf{E}_{\phi} \otimes \mathbf{e}_{\theta}+\frac{l}{\zeta} \mathbf{E}_{\xi} \otimes \mathbf{e}_{z},
$$

where $k=\pi /\left(\pi-\Phi_{0}\right)$. The deformation gradient is diagonal and may be expressed in terms of the principal stretches

$$
\lambda_{r}=\frac{\partial r}{\partial \rho}, \quad \lambda_{\theta}=k \frac{r}{\rho}, \quad \text { and } \quad \lambda_{z}=\lambda \Lambda
$$

where $\lambda=l / L$ is the in situ axial prestretch and $\Lambda=L / \zeta$ is an axial stretch associated with the closing of the cut-open arterial segment. Following Chuong and Fung (1986), the axial closing stretch is typically assumed to be unity, i.e. $\Lambda=1$. The artery is considered incompressible, cf. Section 2.2, and the deformation must, therefore, be volume preserving. Following Eq. (3) the principle stretches must satisfy:

$$
\lambda_{r} \lambda_{\theta} \lambda_{z}=1
$$

The incompressibility constraint in Eq. (51) can be used to find a relation between the referential and deformed radii. Using the principle stretches in Eq. (50) in Eq. (51), one finds after integration over the radius and some straightforward manipulations:

$$
k \lambda \Lambda r^{2}=\rho^{2}+D
$$

where $D=k \lambda \Lambda r_{\mathrm{i}}^{2}-\rho_{\mathrm{i}}^{2}$ is a constant of integration found by inserting the inner radius into Eq. (52). In case of the uniform strain hypothesis (Takamizawa and Hayashi, 1987), $D=0$ as shown in Paper II.

Another implication of incompressibility together with a (constant) in situ axial prestretch is that the current cross-sectional area

$$
A=2 \pi r_{\mathrm{i}} h+\pi h^{2}
$$

of an artery is constant.

\subsubsection{Constitutive equation}

The choice of constitutive equation is fundamental for an appropriate description of arterial tissue since it introduces most of the arterial characteristics into the mechanical model. The concept of hyperelasticity is adopted in this dissertation meaning that the stress-deformation relationship derives from a strain-energy 
function $\Psi$, cf. Section 3.4. Since an artery is assumed to be incompressible, we have according to Eq. (36):

$$
\boldsymbol{\sigma}=-p \mathbf{I}+2 \mathbf{F} \frac{\partial \bar{\Psi}}{\partial \mathbf{C}} \mathbf{F}^{\top}
$$

Many different strain-energy functions have been proposed for vascular tissue. The Holzapfel-Gasser-Ogden (HGO) strain-energy function (Holzapfel et al., 2000) is used in this dissertation to describe the passive response of the tissue. Furthermore, in Paper IV this passive model is extended to account for smooth muscle contraction using the model proposed in Rachev and Hayashi (1999).

\subsubsection{Passive constitutive model}

The HGO model is inspired by the microstructure of the arterial wall as it takes individual constituents and their orientation into account. The HGO strain-energy function is additively decomposed into an isotropic part representing the noncollagenous matrix material, e.g. elastin, and an anisotropic part associated with the embedded collagen fibers. The isotropic part is expressed in terms of the classical neo-Hookean model (Treloar, 1943):

$$
\bar{\Psi}^{\text {iso }}=c\left(I_{1}-3\right),
$$

where $c>0$ is a stress-like material parameter. Following Schriefl et al. (2012), all embedded collagen fibers are taken to belong to one of two fiber families symmetrically arranged around the circumferential direction with the pitch angle $\pm \beta$ in the reference configuration, see Figure 12. The two fiber families are assumed to be mechanically equivalent and their orientation is described by the two referential unit vectors

$$
\mathbf{M}=\cos \beta \mathbf{E}_{\phi}+\sin \beta \mathbf{E}_{\xi} \quad \text { and } \quad \mathbf{N}=\cos \beta \mathbf{E}_{\phi}-\sin \beta \mathbf{E}_{\xi} .
$$

The anisotropic part of the strain-energy function associated with the two collagen fiber families is given by

$$
\bar{\Psi}^{\text {aniso }}=\frac{k_{1}}{2 k_{2}}\left(\mathrm{e}^{k_{2}\left(I_{4}-1\right)^{2}}+\mathrm{e}^{k_{2}\left(I_{6}-1\right)^{2}}-2\right),
$$

where $k_{1}>0$ is a stress-like material parameter and $k_{2}>0$ is a dimensionless parameter. It holds by Eqs. (40), (42), (49), (50), and (56) that

$$
I_{4}=I_{6}=\lambda_{\theta}^{2} \cos ^{2} \beta+\lambda_{z}^{2} \sin ^{2} \beta .
$$

The collagen fibers are assumed to only support tensile loads and buckle in compression (Holzapfel et al., 2000). Recalling that $I_{4}$ and $I_{6}$ are equal to the squared stretch of their respective fiber family, cf. Section 3.4.2, the anisotropic contribution $\bar{\Psi}^{\text {aniso }}$ is therefore omitted if $I_{4}, I_{6}<1$, i.e.

$$
\bar{\Psi}^{\text {pas }}= \begin{cases}\bar{\Psi}^{\text {iso }}+\bar{\Psi}^{\text {aniso }} & \text { if } I_{4}, I_{6} \geq 1 \\ \bar{\Psi}^{\text {iso }} & \text { otherwise }\end{cases}
$$


Using the additive split of the HGO strain-energy function, it is also possible to decompose the isochoric part of the Cauchy stress tensor in Eq. (54) into two parts: an isotropic and an anisotropic stress defined by

$$
\overline{\boldsymbol{\sigma}}^{\text {iso }}=2 \mathbf{F} \frac{\partial \bar{\Psi}^{\text {iso }}}{\partial \mathbf{C}} \mathbf{F}^{\top} \text { and } \overline{\boldsymbol{\sigma}}^{\text {aniso }}=2 \mathbf{F} \frac{\partial \bar{\Psi}^{\text {aniso }}}{\partial \mathbf{C}} \mathbf{F}^{\top},
$$

respectively. By combining Eqs. (54), (59), and (60), the following constitutive equation for a passive artery is obtained:

$$
\boldsymbol{\sigma}= \begin{cases}-p \mathbf{I}+\overline{\boldsymbol{\sigma}}^{\text {iso }}+\overline{\boldsymbol{\sigma}}^{\text {aniso }} & \text { if } I_{4}, I_{6} \geq 1 \\ -p \mathbf{I}+\overline{\boldsymbol{\sigma}}^{\text {iso }} & \text { otherwise }\end{cases}
$$

The HGO model is the basis for more sophisticated (passive) models which account for a distribution of the collagen fibers along the preferred direction (Gasser et al., 2006, Holzapfel et al., 2015). These models reflect the microstructure of the arterial wall more accurately, but introduce additional parameters associated with the collagen distribution and are, therefore, less suited for an in vivo parameter identification method.

\subsubsection{Active constitutive model}

The ability of smooth muscle cells to actively contract is introduced into the mechanical model of an artery through the constitutive equation by adding an active stress $\overline{\boldsymbol{\sigma}}^{\text {act }}$ to Eq. (54) (Hill, 1938, Rachev and Hayashi, 1999):

$$
\boldsymbol{\sigma}=-p \mathbf{I}+2 \mathbf{F} \frac{\partial \bar{\Psi}}{\partial \mathbf{C}} \mathbf{F}^{\top}+\overline{\boldsymbol{\sigma}}^{\text {act }}
$$

The HGO strain-energy function introduced in Section 4.1.2.1 is used for the passive response of an artery, i.e. $\bar{\Psi}=\bar{\Psi}^{\text {pas }}$. As pointed out in Section 2.2 , smooth muscle cells contract slowly but once contracted maintain this state for long periods of time. Hence, it is assumed that the contracted state does not change during a cardiac cycle. Furthermore, smooth muscle cells are primarily oriented in the circumferential direction, see Section 2.2, and following Rachev and Hayashi (1999) the active stress is therefore computed as

$$
\overline{\boldsymbol{\sigma}}^{\text {act }}=\hat{S} \lambda_{\theta} \mathbf{e}_{\theta} \otimes \mathbf{e}_{\theta},
$$

where $\hat{S}>0$ is a parameter associated with the stiffness provided by the contracted smooth muscle cells, cf. the latch-bridges in Section 2.3. The parameter $\hat{S}$ depends on the contracted state of the smooth muscle cells, hence on the length-tension relationship, the tension-velocity relationship, and the degree of smooth muscle activation, cf. Section 2.3. Here only the dependence on the length-tension relationship is made explicit:

$$
\hat{S}=S f\left(\bar{\lambda}_{\theta}\right)
$$




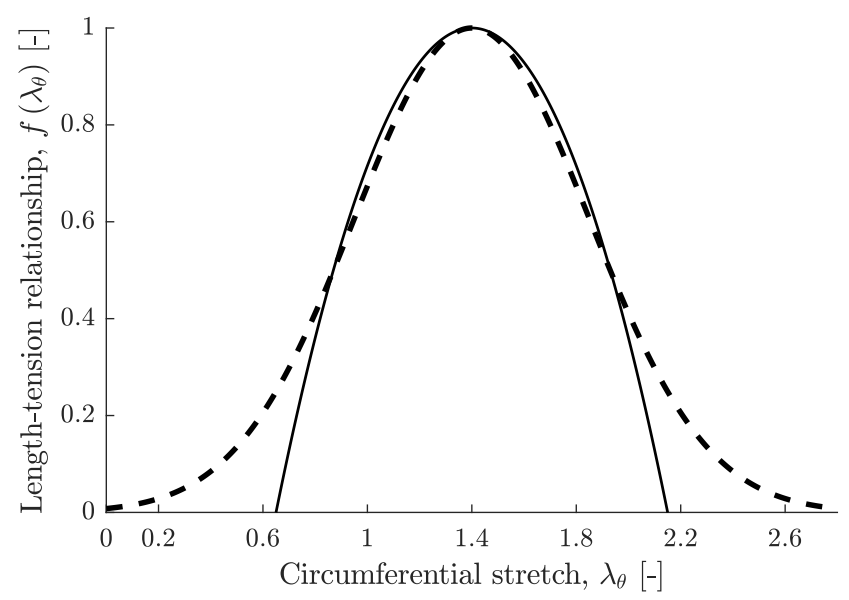

Figure 13: Length-tension relationship of smooth muscle cells. The solid and dashed lines denote the parabola suggested in Rachev and Hayashi (1999) and its parabola-like exponential representation in Eq. (65), with $\omega=0.45$, respectively.

where $S>0$ is the generated isometric stress per unit reference area ${ }^{2}, f\left(\lambda_{\theta}\right)>0$ is a function describing the length-tension relationship of smooth muscle cells with $\max f\left(\lambda_{\theta}\right)=1$, and $\bar{\lambda}_{\theta}$ is the stretch at which the smooth muscle cells contracted ${ }^{3}$.

As written in Section 2.3 the length-tension relationship of smooth muscle cells has a parabolic shape and in order to satisfy $f\left(\lambda_{\theta}\right)>0$ the following parabolic-like exponential function is used:

$$
f\left(\lambda_{\theta}\right)=\exp \left[-\frac{\left(\lambda_{\theta}^{\mathrm{opt}}-\lambda_{\theta}\right)^{2}}{2 \omega^{2}}\right],
$$

where $\lambda_{\theta}^{\text {opt }}=1.4$ is the optimal stretch for maximum force generation (Rachev and Hayashi, 1999) and $\omega$ controls the width of the parabola-like exponential function. Here $\omega=0.45$ is used such that the length-tension relationship in Eq. (65) resembles the parabola suggested in Rachev and Hayashi (1999) well in the interval $0.8 \leq \lambda_{\theta} \leq 1.4$, see Figure 13 .

The presented extension to account for smooth muscle contraction is simple and only performs adequately when smooth muscle cells do not change their contracted state. More advanced active models have been proposed to study smooth muscle contraction (Kroon, 2009, Murtada et al., 2012, Schmitz and Böl, 2011, Stålhand et al., 2011, Zulliger et al., 2004). These advanced models require many parameters

\footnotetext{
${ }^{2}$ In Paper IV, $S=0$ is used to provide an easy-to-understand means to reduce the active model to the passive model.

${ }^{3}$ In the original work the length-tension relationship is not evaluated at a specific stretch but used as function of the smooth muscle stretch instead (Rachev and Hayashi, 1999).
} 
and are, therefore, less suited for an in vivo parameter identification method. For the presented active model only one parameter, namely $S$, needs to be identified.

\subsubsection{Boundary conditions}

An artery is exposed to the flow of pressurized blood from inside the lumen, constrained by its surrounding from the outside, and axially prestreched in situ, cf. Section 2.2. Since short time spans are considered and, therefore, growth and remodeling do not need to be accounted for, shear stress can also be neglected as it is several orders of magnitude smaller than the normal stress caused by the blood pressure, cf. Section 2.2. Furthermore, the outside of an artery is assumed to be traction free, since information about the perivascular state is not included in the used data sets. Using a population averaged (De Keulenaer et al., 2009, Guyton and Hall, 2006) or identifying a patient-specific perivascular pressure (Humphrey and Na, 2002, Singh and Devi, 1990) were considered, but these ideas were ultimately discarded. As a consequence, the transmural stress state is slightly overestimated, see Figure 5.

Summarizing the loading situation of an artery, it is assumed that the artery is prestretched by a constant $\lambda$ in the axial direction and exposed to a pressure $P=P(t)$ on the inside with zero traction on the outside, i.e. the radial stress is

$$
\sigma_{r r}= \begin{cases}-P & \text { on } r=r_{\mathrm{i}}, \\ 0 & \text { on } r=r_{\mathrm{o}}\end{cases}
$$

\subsubsection{Equilibrium}

Arterial tissue is typically assumed to be in quasi-static equilibrium in vivo. Despite this simplification of the equation of motion, studies suggest that this choice is appropriate because inertia appears to only play a minor role (Chaudhry et al., 1997, Demiray and Vito, 1983, Humphrey, 2002, Singh and Devi, 1990). Dropping the inertia term and further neglecting body forces, the equation of motion in Eq. (14) reduces to the equilibrium equation

$$
\operatorname{div} \boldsymbol{\sigma}=\mathbf{0}
$$

Equations (49) and (54) together with either the passive constitutive model in Section 4.1.2.1 or its active extension in Section 4.1.2.2 yield a shear-free stress field and the only non-trivial component of Eq. (67) in cylindrical coordinates is in the radial direction ${ }^{4}$ :

$$
\frac{\partial \sigma_{r r}}{\partial r}+\frac{1}{r}\left(\sigma_{r r}-\sigma_{\theta \theta}\right)=0
$$

\footnotetext{
${ }^{4} \mathrm{~A}$ complete derivation of the equilibrium equation in cylindrical coordinates can be found in Volokh (2006).
} 
Integrating Eq. (68) from the inner radius $r_{\mathrm{i}}$ to the radial coordinate $r$, the radial stress becomes

$$
\sigma_{r r}(r)=\sigma_{r r}\left(r_{\mathrm{i}}\right)+\int_{r_{\mathrm{i}}}^{r}\left(\sigma_{\theta \theta}-\sigma_{r r}\right) \frac{\mathrm{d} r}{r} .
$$

Applying the boundary conditions in Eq. (66), the transmural pressure is obtained as

$$
P=\int_{r_{\mathrm{i}}}^{r_{\mathrm{o}}}\left(\sigma_{\theta \theta}-\sigma_{r r}\right) \frac{\mathrm{d} r}{r}=\int_{r_{\mathrm{i}}}^{r_{\mathrm{o}}}\left(\bar{\sigma}_{\theta \theta}-\bar{\sigma}_{r r}\right) \frac{\mathrm{d} r}{r},
$$

where the latter equality follows from Eq. (36).

In addition, the axial force $N$ is calculated from equilibrium in the axial direction as

$$
N=2 \pi \int_{r_{\mathrm{i}}}^{r_{\mathrm{o}}} \sigma_{z z} r \mathrm{~d} r
$$

This is not the force measured in in vitro experiments where the blood pressure acts on the closed ends and, therefore, reduces the force necessary to hold the artery in place. The reduced axial force is given by

$$
F_{\text {red }}=2 \pi \int_{r_{\mathrm{i}}}^{r_{\mathrm{o}}} \sigma_{z z} r \mathrm{~d} r-\pi P r_{\mathrm{i}}^{2}
$$

and has been reported to be approximately constant throughout the cardiac cycle at the in situ axial prestretch, cf. Section 2.2.

Furthermore, the Lagrange multiplier in Eq. (54) can be calculated by combining Eqs. (54), (66), and (69) to get:

$$
p(r)=P+\bar{\sigma}_{r r}(r)-\int_{r_{\mathrm{i}}}^{r}\left(\bar{\sigma}_{\theta \theta}-\bar{\sigma}_{r r}\right) \frac{\mathrm{d} r}{r} .
$$

\subsection{Constitutive membrane model}

The constitutive membrane model is an arterial model specialized for the in vivo parameter identification method and based upon the model proposed in Stålhand (2009). It is a simplified version of the general model in Section 4.1. The main difference lies in the treatment of an artery as a membrane instead of a thick-walled structure. Neglecting transmural dependencies allows for the analytical treatment of the governing equations and no numerical solution techniques need to be employed.

Two sets of stresses are required by the in vivo parameter identification method for the constitutive membrane model: equilibrium and constitutively determined stresses.

Depending on whether the constitutively determined stresses are calculated with the passive constitutive model in Section 4.1.2.1 or its active extension in Section 4.1.2.2, the constitutive membrane model is referred to as passive or active, respectively. 


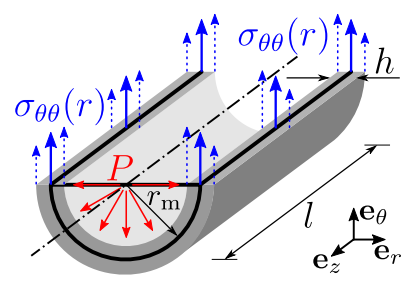

(a) Diametral-section cut

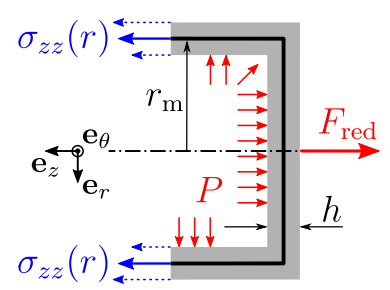

(b) Cross-section cut

Figure 14: Cuts of a vessel with capped ends subjected to a blood pressure $P$ and a pressure-independent axial force $\bar{F}_{\text {red }}$. The solid black lines and the grey area represent the thin-walled and the thick-walled vessel, respectively.

\subsubsection{Equilibrium stresses}

The stress state of an artery subjected to a blood pressure ${ }^{5} P$ and a constant reduced axial force $F_{\text {red }}$ is calculated by enforcing global equilibrium. Stating equilibrium, one gets in the circumferential direction, cf. Figure 14a:

$$
-2 P l\left(r_{\mathrm{m}}-\frac{h}{2}\right)+2 l \int_{r_{\mathrm{m}}-\frac{h}{2}}^{r_{\mathrm{m}}+\frac{h}{2}} \sigma_{\theta \theta} \mathrm{d} r=0,
$$

where $r_{\mathrm{m}}$ denotes the deformed mean radius, and in the axial direction, cf. Figure 14b:

$$
-P \pi\left(r_{\mathrm{m}}-\frac{h}{2}\right)^{2}-F_{\text {red }}+\int_{r_{\mathrm{m}}-\frac{h}{2}}^{r_{\mathrm{m}}+\frac{h}{2}} \sigma_{z z} 2 \pi r \mathrm{~d} r=0 .
$$

Assuming that both the circumferential and axial stress are constant through the thickness, i.e. independent of $r$, they are calculated by using Eqs. (74) and (75) as:

$$
\sigma_{\theta \theta}^{\mathrm{Lp}}=\frac{r_{\mathrm{m}}-0.5 h}{h} P=\left(\frac{r_{\mathrm{i}}}{h}+\alpha\right) P
$$

and

$$
\sigma_{z z}^{\mathrm{Lp}}=\frac{\pi r_{\mathrm{i}}^{2} P+F_{\mathrm{red}}}{\pi h\left(2 r_{\mathrm{i}}+h\right)}
$$

where the superscript Lp denotes Laplace's law and $\alpha=0$. In Papers I, II, and III the circumferential stress is overestimated by half the blood pressure. This is achieved by using $\alpha=0.5$ in Eq. (76) and is equivalent to the thin-walled simplification $r_{\mathrm{m}}-h / 2 \approx r_{\mathrm{m}}$ based on $r_{\mathrm{m}} \gg h$ (Ugural, 1999).

The reduced axial force $F_{\text {red }}$ cannot be measured in vivo. Following SchulzeBauer and Holzapfel (2003) this force is therefore estimated by assuming that the

\footnotetext{
${ }^{5}$ In case information about the perivascular pressure is available, it can be accounted for by simply replacing the blood pressure by the transmural pressure.
} 
ratio $\gamma$ between the axial and circumferential stress is $\gamma=0.59$ at MAP. Combining this with Eqs. (76) and (77) gives the estimated reduced axial force

$$
\bar{F}_{\text {red }}=P^{\mathrm{MAP}} \pi\left[\gamma\left(r_{\mathrm{i}}^{\mathrm{MAP}}+\alpha h^{\mathrm{MAP}}\right)\left(2 r_{\mathrm{i}}^{\mathrm{MAP}}+h^{\mathrm{MAP}}\right)-\left(r_{\mathrm{i}}^{\mathrm{MAP}}\right)^{2}\right],
$$

where the superscript MAP denotes a quantity evaluated at MAP.

The radial stress $\sigma_{r r}$ varies from the applied blood pressure $P$ on the inner boundary to zero on the outer boundary, cf. Eq. (66), and is thus much smaller compared to the membrane stresses in Eqs. (76) and (77). The radial stress is, therefore, neglected and specified as zero, i.e.

$$
\sigma_{r r}^{\mathrm{Lp}}=0 .
$$

\subsubsection{Constitutively determined stresses}

To be able to use the constitutive equation in Eq. (54), the deformation gradient $\mathbf{F}^{\text {mod }}$ associated with the constitutive membrane model needs to be established.

The artery is modeled as a homogeneous, incompressible, residual-stress free, and thin-walled tube. The corresponding stress-free reference configuration is the unloaded configuration $\mathscr{B}^{*}$ in Figure 12 with mean referential radius $R_{\mathrm{m}}$, wall thickness $H$, and axial length $L$. Since residual stress is neglected the deformation gradient in Eq. (46) associated with the deformation between the reference and the deformed configuration reduces to $\mathbf{F}_{\mathrm{e}}$. In addition, the principal stretches in Eq. (50) are no longer functions of the radial position due to the thin-walled assumption. Let the artery be prestretched in situ by a constant $\lambda$ in the axial direction; the circumferential stretch be defined in the mid-wall according to

$$
\lambda_{\theta, \mathrm{m}}=\frac{r_{\mathrm{o}}+r_{\mathrm{i}}}{R_{\mathrm{o}}+R_{\mathrm{i}}}=\frac{2 r_{\mathrm{i}}+h}{R_{\mathrm{i}}+\sqrt{R_{\mathrm{i}}^{2}+\lambda h\left(2 r_{\mathrm{i}}+h\right)}} ;
$$

and the radial stretch is then given implicitly by the incompressibility constraint in Eq. (51). The deformation gradient for the constitutive membrane model is thus

$$
\mathbf{F}^{\mathrm{mod}}=\left(\lambda_{\theta, \mathrm{m}} \lambda\right)^{-1} \mathbf{e}_{r} \otimes \mathbf{e}_{r}+\lambda_{\theta, \mathrm{m}} \mathbf{e}_{\theta} \otimes \mathbf{e}_{\theta}+\lambda \mathbf{e}_{z} \otimes \mathbf{e}_{z} .
$$

As in Eq. (79) the radial model stress is set to $\sigma_{r r}^{\text {pas }}=0$ and the Lagrange multiplier $p$ arising from the incompressibility constraint can be calculated from the radial component in Eq. (54) as

$$
p^{\bmod }=2 c\left(\lambda_{\theta, \mathrm{m}} \lambda\right)^{-2} .
$$

Back-substitution of the result into Eq. (54) and using Eqs. (40), (42), and (55) to (59) with the deformation gradient $\mathbf{F}^{\text {mod }}$ in Eq. (81), give the passive constitutively determined stresses in the circumferential direction:

$$
\sigma_{\theta \theta}^{\mathrm{pas}}= \begin{cases}2 c\left[\lambda_{\theta, \mathrm{m}}^{2}-\left(\lambda_{\theta, \mathrm{m}} \lambda\right)^{-2}\right]+\ldots & \text { if } I_{4} \geq 1, \\ 4 k_{1}\left(I_{4}-1\right) \lambda_{\theta, \mathrm{m}}^{2} \cos ^{2} \beta \mathrm{e}^{k_{2}\left(I_{4}-1\right)^{2}} & \\ 2 c\left[\lambda_{\theta, \mathrm{m}}^{2}-\left(\lambda_{\theta, \mathrm{m}} \lambda\right)^{-2}\right] & \text { otherwise }\end{cases}
$$


and in the axial direction:

$$
\sigma_{z z}^{\mathrm{pas}}= \begin{cases}2 c\left[\lambda^{2}-\left(\lambda_{\theta, \mathrm{m}} \lambda\right)^{-2}\right]+\ldots & , \text { if } I_{4} \geq 1 \\ 4 k_{1}\left(I_{4}-1\right) \lambda^{2} \sin ^{2} \beta \mathrm{e}^{k_{2}\left(I_{4}-1\right)^{2}} & \\ 2 c\left[\lambda^{2}-\left(\lambda_{\theta, \mathrm{m}} \lambda\right)^{-2}\right] & \text { otherwise. }\end{cases}
$$

By analogy with Eq. (60), the isochoric stress can be decomposed into an isotropic and an anisotropic part in the constitutive membrane model.

Equations (83) and (84) are the constitutively determined stresses of the passive constitutive membrane model used in Papers I, II, and III.

In case of the active constitutive membrane model, which is used in Paper IV, the active stress given in Eqs. (63) to (65), with $\lambda_{\theta}$ replaced by Eq. (80), is simply added to Eq. (83) and together with Eq. (84) they represent the constitutively determined stresses, i.e. in the circumferential direction:

$$
\sigma_{\theta \theta}^{\text {act }}=\left\{\begin{array}{cc}
2 c\left[\lambda_{\theta, \mathrm{m}}^{2}-\left(\lambda_{\theta, \mathrm{m}} \lambda\right)^{-2}\right]+S \lambda_{\theta, \mathrm{m}} f\left(\bar{\lambda}_{\theta, \mathrm{m}}\right)+\ldots & , \text { if } I_{4} \geq 1, \\
4 k_{1}\left(I_{4}-1\right) \lambda_{\theta, \mathrm{m}}^{2} \cos ^{2} \beta \mathrm{e}^{k_{2}\left(I_{4}-1\right)^{2}} & \text {, otherwise } \\
2 c\left[\lambda_{\theta, \mathrm{m}}^{2}-\left(\lambda_{\theta, \mathrm{m}} \lambda\right)^{-2}\right]+S \lambda_{\theta, \mathrm{m}} f\left(\bar{\lambda}_{\theta, \mathrm{m}}\right) & \text {, }
\end{array}\right.
$$

and in the axial direction:

$$
\sigma_{z z}^{\text {act }}= \begin{cases}2 c\left[\lambda^{2}-\left(\lambda_{\theta, \mathrm{m}} \lambda\right)^{-2}\right]+\ldots & \\ 4 k_{1}\left(I_{4}-1\right) \lambda^{2} \sin ^{2} \beta \mathrm{e}^{k_{2}\left(I_{4}-1\right)^{2}} & , \text { if } I_{4} \geq 1 \\ 2 c\left[\lambda^{2}-\left(\lambda_{\theta, \mathrm{m}} \lambda\right)^{-2}\right] & , \text { otherwise }\end{cases}
$$





\section{Parameter Identification}

The constitutive membrane model is developed in Section 4.2 to approximate arterial tissue. It includes a number of unknown model parameter which are related to the mechanical properties of the tissue. These parameters need to be determined given the measurable data which is generally limited to time-resolved blood pressure and radial deformation, cf. Figure $3 \mathrm{~b}$.

On a more general level the constitutive membrane model constitutes a parameterized response function approximating arterial tissue. The model parameters cannot be measured directly but must be determined by a parameter identification. Within the parameter identification the model parameters are tuned such that the response function is able to explain the observed measurements. Consider therefore the measured blood pressure $P$ exciting the tissue and causing a deformation in terms of the inner radius $r_{\mathrm{i}}$. Given this data, the inverse problem to identify the model parameters, which are collected in the vector $\boldsymbol{\kappa}$, consists in solving the non-linear equations ${ }^{1}$

(IIP) $r_{\mathrm{i}}^{\bmod }\left(\boldsymbol{\kappa}, P_{j}\right)=r_{\mathrm{i}, j}$,

where $r_{\mathrm{i}}^{\text {mod }}$ is the model's response function to predict the inner radius, $j=1, \ldots, n$ is a measurement sample, and $n$ is the total number of measurements.

The inverse problem $(\mathbb{I P})$ does not have a solution since measurement errors exist in general and in this particular case the response function cannot account for the hysteresis present in the pressure-radius data, see Figure 3b, because it is not a function of time. By rewriting the inverse problem as an optimization problem, however, in which the differences between the measurements and the model predictions are minimized, the problem can be solved (Mahnken, 2004). The optimization problem is typically formulated as a least-squares minimization problem according to

$(\mathbb{M P}) \underset{\kappa}{\operatorname{minimize}} \sum_{j=1}^{n}\left[r_{\mathrm{i}, j}-r_{\mathrm{i}}^{\bmod }\left(\boldsymbol{\kappa}, P_{j}\right)\right]^{2}$.

Depending on the response function and the number of model parameters, the minimization problem $(\mathbb{M P})$ might not have a unique solution but is instead

\footnotetext{
${ }^{1}$ More common is to reverse the causality and reformulate the problem in terms of the blood pressure because it is easier to calculate the blood pressure given a deformation than vice versa, cf. Eq. (70).
} 
overparameterized, i.e. there are many different model parameter combinations which solve $(\mathbb{M P})$ equally well. It is, therefore, not possible to select one set of model parameters over another (Mahnken, 2004).

Overparameterization can be prevented by including measurements exciting other modes ${ }^{2}$ of the model, introducing constraints, and if the former are not sufficient, the response function must ultimately be simplified. Common constraints are to add lower and upper bounds on the model parameters and to constrain the response function to a behavior which has been observed in experiments. A specific constraint, which is frequently used in the parameter identification for arteries, is to require that the reduced axial force is constant throughout the cardiac cycle at the in situ axial prestretch $\lambda$, i.e.

$$
\left.\frac{\partial F_{\text {red }}}{\partial P}\right|_{\lambda}=0
$$

cf. Section 2.2. An even stronger version of this constraint is to require that the predicted reduced axial force $F_{\text {red }}^{\text {mod }}$ is equal to some estimated value. Denoting the estimated reduced axial force by $\bar{F}_{\text {red }}$, cf. Section 4.2 .1 , the corresponding constraint reads:

$$
F_{\text {red }}^{\text {mod }}\left(\boldsymbol{\kappa}, P_{j}\right)=\bar{F}_{\text {red }} .
$$

Equality constraints, such as in Eqs. (87) and (88), restrict the solution to a hypersurface in the solution space. A solution, however, only exists if the response function allows for the constraint behavior in the first place. To relax the constraint, the deviation of the constraint behavior is instead penalized, e.g. in the least-squares sense. The corresponding minimization problem has now two objectives: minimize the least-squares differences of measured and predicted inner radius; and minimize the deviation from the constraint behavior. One way to solve this multi-objective minimization problem is to minimize the sum of the individual objectives, thus making it a single-objective minimization problem again. Using the reduced axial force constraint in Eq. (88) and introducing box constraints on the model parameters, the constrained minimization problem becomes

$(\mathbb{C M P})\left\{\begin{array}{l}\underset{\kappa}{\operatorname{minimize}} \sum_{j=1}^{n}\left\{\left[r_{\mathrm{i}, j}-r_{\mathrm{i}}^{\bmod }\left(\boldsymbol{\kappa}, P_{j}\right)\right]^{2}+\left[\bar{F}_{\text {red }}-F_{\text {red }}^{\bmod }\left(\boldsymbol{\kappa}, P_{j}\right)\right]^{2}\right\}, \\ \text { subject to: } \boldsymbol{\kappa}^{\min } \leq \boldsymbol{\kappa} \leq \boldsymbol{\kappa}^{\max }\end{array}\right.$

where $\boldsymbol{\kappa}^{\min }$ and $\boldsymbol{\kappa}^{\max }$ denote the lower and upper bound of $\boldsymbol{\kappa}$, respectively. Depending on the magnitude of the individual objectives one of them will dominate the solution. By introducing weighting factors for the individual objectives, e.g. $w_{1}$ for the deformation and $w_{2}$ for the reduced axial force in $(\mathbb{C M P})$, the influence of the individual objectives can be controlled. A closely related topic is normalization in

\footnotetext{
${ }^{2}$ For example, the minimization problem $(\mathbb{M P})$ considers only measurements in the radialcircumferential plane. The behavior of the model in the axial direction is not controlled.
} 
which the magnitude of the individual objectives are normalized such that they influence the minimization equally. More details about normalization are found in Section 5.1. Another very important aspect concerns solving the minimization problem numerically and this topic is discussed in Section 5.2. To shorten the notation, the scalar-valued function $\varepsilon$ is introduced denoting the objective function, e.g. for $(\mathbb{C M P})$ :

$$
\varepsilon(\boldsymbol{\kappa})=\sum_{j=1}^{n}\left\{\left[r_{\mathrm{i}, j}-r_{\mathrm{i}}^{\mathrm{mod}}\left(\boldsymbol{\kappa}, P_{j}\right)\right]^{2}+\left[\bar{F}_{\text {red }}-F_{\text {red }}^{\bmod }\left(\boldsymbol{\kappa}, P_{j}\right)\right]^{2}\right\} .
$$

The objective function or sometimes called the error function depends on the measurements as well, cf. Eq. (89). For convenience, this dependency is omitted throughout.

\subsection{Normalization}

Normalization is explained with respect to the objective function in Eq. (89). For this particular objective function, the goal is generally not to weigh both parts equally but to weigh the reduced axial force part just enough such that the arterial model behaves realistically. The reduced axial force part in Eq. (89) is therefore used as a regularization (Smoljkić et al., 2015, Spronck et al., 2015, Stålhand and Klarbring, 2005). For the remainder of this section it is assumed that both parts in Eq. (89) are supposed to have an equal effect on the objective function, however.

Several approaches have been proposed in the literature to normalize an objective function containing multiple objectives. In the following three common approaches are introduced.

Normalization 1: The first normalization approach has already been mentioned and concerns the addition of weighting factors for the individual objectives. The weighted objective function reads:

$$
\varepsilon(\boldsymbol{\kappa})=\sum_{j=1}^{n}\left\{w_{1}\left[r_{\mathrm{i}, j}-r_{\mathrm{i}}^{\mathrm{mod}}\left(\boldsymbol{\kappa}, P_{j}\right)\right]^{2}+w_{2}\left[\bar{F}_{\text {red }}-F_{\text {red }}^{\bmod }\left(\boldsymbol{\kappa}, P_{j}\right)\right]^{2}\right\},
$$

where the weights $w_{1}$ and $w_{2}$ are chosen such that the individual objectives are equally weighted. In the literature there is no consensus about how these weights should be selected (Mausser, 2006). One common choice however is to equalize the magnitude of the measurements, e.g. if the inner radius is in the range of $5-10 \mathrm{~mm}$ and the reduced axial force ${ }^{3}$ around $1000 \mathrm{mN}$ favorable weights could be $w_{1}=1$ and $w_{2}=2 \cdot 10^{-2}$. A second common choice is to set the weights equal to one divided by the average value of the respective measurement, i.e.

$$
w_{1}=n\left(\sum_{j=1}^{n} r_{\mathrm{i}, j}\right)^{-1} \text { and } w_{2}=\bar{F}_{\text {red }}^{-1} .
$$

\footnotetext{
${ }^{3}$ Forces are measured in $\mathrm{mN}$ if a length is measured in $\mathrm{mm}$, time in $\mathrm{s}$, and mass in $\mathrm{kg}$. This unit-system is used if stress-like quantities are supposed to be given in $\mathrm{kPa}$.
} 
Normalization 2: The second normalization approach employs, so called, Utopia and Nadir points. In this approach the normalized objective function reads (Mausser, 2006):

$$
\varepsilon(\boldsymbol{\kappa})=\sum_{q=1}^{2} \frac{\varepsilon_{q}(\boldsymbol{\kappa})-\varepsilon_{q}^{\text {Utopia }}}{\varepsilon_{q}^{\text {Nadir }}-\varepsilon_{q}^{\text {Utopia }}},
$$

where $q=1,2$,

$$
\begin{aligned}
& \varepsilon_{1}(\boldsymbol{\kappa})=\sum_{j=1}^{n}\left[r_{\mathrm{i}, j}-r_{\mathrm{i}}^{\bmod }\left(\boldsymbol{\kappa}, P_{j}\right)\right]^{2}, \\
& \varepsilon_{2}(\boldsymbol{\kappa})=\sum_{j=1}^{n}\left[\bar{F}_{\text {red }}-F_{\text {red }}^{\bmod }\left(\boldsymbol{\kappa}, P_{j}\right)\right]^{2},
\end{aligned}
$$

and $\varepsilon_{q}^{\text {Utopia }}$ and $\varepsilon_{q}^{\text {Nadir }}$ are the Utopia and Nadir points, respectively, of the individual objectives in Eqs. (93) and (94). The Utopia point of objective $q$ is the lowest objective function value if only that objective is considered, i.e.

$$
\varepsilon_{q}^{\mathrm{Utopia}}=\underset{\kappa}{\operatorname{minimize}} \varepsilon_{q}(\boldsymbol{\kappa}),
$$

where $\boldsymbol{\kappa} \in\left[\boldsymbol{\kappa}^{\min }, \boldsymbol{\kappa}^{\max }\right]$. The Nadir point of objective $q$ is conversely the objective function value when the parameter vector of another Utopia point is used to evaluate $\varepsilon_{q}$, e.g. the Nadir point of objective $q=1$ is:

$$
\varepsilon_{1}^{\mathrm{Nadir}}=\varepsilon_{1}\left(\boldsymbol{\kappa}_{2}^{*}\right)
$$

where $\boldsymbol{\kappa}_{2}^{*}$ is the parameter vector of $\varepsilon_{2}^{\text {Utopia }}$. The Utopia and Nadir points are, therefore, best-case and worst-case values and it is easy to see that each individual objective in Eq. (92) satisfies:

$$
0 \leq \frac{\varepsilon_{q}(\boldsymbol{\kappa})-\varepsilon_{q}^{\text {Utopia }}}{\varepsilon_{q}^{\text {Nadir }}-\varepsilon_{q}^{\text {Utopia }}} \leq 1,
$$

providing equal weight to each individual objective.

Normalization 3: The third normalization approach concerns rewriting the original objective function in such a way that the individual objectives are implicitly normalized. In the context of parameter identification for arteries, it is suggested in Schulze-Bauer and Holzapfel (2003) to convert the pressure-radius measurements and the (estimated) reduced axial force into corresponding stresses in the circumferential, axial, and radial direction using the equilibrium equation (67). The normalized 
objective function is:

$$
\begin{gathered}
\varepsilon(\boldsymbol{\kappa})=\sum_{j=1}^{n}\left\{\left[\sigma_{\theta \theta}^{\mathrm{Eq}}\left(P_{j}, r_{\mathrm{i}, j}\right)-\sigma_{\theta \theta}^{\bmod }\left(\boldsymbol{\kappa}, P_{j}, r_{\mathrm{i}, j}\right)\right]^{2}+\ldots\right. \\
{\left[\sigma_{z z}^{\mathrm{Eq}}\left(P_{j}, r_{\mathrm{i}, j}\right)-\sigma_{z z}^{\bmod }\left(\boldsymbol{\kappa}, P_{j}, r_{\mathrm{i}, j}\right)\right]^{2}+\ldots} \\
\\
\left.\left[\sigma_{r r}^{\mathrm{Eq}}\left(P_{j}, r_{\mathrm{i}, j}\right)-\sigma_{r r}^{\bmod }\left(\boldsymbol{\kappa}, P_{j}, r_{\mathrm{i}, j}\right)\right]^{2}\right\}
\end{gathered}
$$

where the superscript Eq denotes equilibrium. This normalization approach is particularly useful if the equilibrium stresses $\sigma_{\theta \theta}^{\mathrm{Eq}}, \sigma_{z z}^{\mathrm{Eq}}$, and $\sigma_{r r}^{\mathrm{Eq}}$ are easily calculated, e.g. for a thin-walled tube using Eqs. (76), (77), and (79).

Although the single objectives in Eq. (98) describe now analogous quantities, the three parts do not necessarily contribute equally to the objective function and an additional normalization using e.g. Normalization 1 and 2 should be considered.

\subsection{Solving the minimization problem numerically}

Deterministic optimization algorithms are the gold standard to solve deterministic minimization problems as are dealt with in this dissertation. These algorithms start from an estimate of the model parameters, identify a direction in which the objective function decreases, update the model parameters with respect to the identified direction, and repeat these steps until the objective function does not decrease in any direction any more, i.e. a stationary point is found. Since the gradient of a function renders the function's slope, at a stationary point the gradient of the objective function is the zero vector and this constitutes the first-order necessary condition for a minimum of an unconstrained minimization problem. Furthermore, if the Hessian at the solution is positive definite, i.e. all eigenvalues of the Hessian are positive, the found stationary point is truly a minimizer of the objective function since the objective function does not only remain the same but increases in all directions (second-order sufficient condition). If the Hessian is instead positive-semidefinite, i.e. one or more eigenvalues are zero and the Hessian has not full rank, the solution is non-unique and there exists a sequence of parameter combinations for which the objective function value remains the same, i.e. the problem is overparameterized.

For constrained minimization problems the first-order necessary condition does not only involve the gradient of the objective function, but also the constraints must be fulfilled at the solution. Before the conditions are stated, the Lagrangian of the objective function is introduced:

$$
\mathcal{L}(\boldsymbol{\kappa}, \boldsymbol{p})=\varepsilon(\boldsymbol{\kappa})+\sum_{l} p_{l} C_{l}(\boldsymbol{\kappa}),
$$

where $\boldsymbol{p}$ is the vector of Lagrange multipliers $p_{l}, l \in \mathcal{E} \cup \mathcal{I}, \mathcal{E}$ represents all indices associated with equality constraints of the form $C(\boldsymbol{\kappa})=0$, and $\mathcal{I}$ denotes all indices 
associated with inequality constraints of the form $C(\boldsymbol{\kappa}) \leq 0$. Assuming sufficient regularity of the feasible set ${ }^{4}$, the first-order necessary conditions for a constrained minimization problem are:

$$
\begin{aligned}
\nabla_{\boldsymbol{\kappa}} \mathcal{L}\left(\boldsymbol{\kappa}^{*}, \boldsymbol{p}^{*}\right) & =\mathbf{0}, \\
C_{l}\left(\boldsymbol{\kappa}^{*}\right) & =0, \text { for all } l \in \mathcal{E}, \\
C_{l}\left(\boldsymbol{\kappa}^{*}\right) & \leq 0, \text { for all } l \in \mathcal{I}, \\
p_{l}^{*} & \geq 0, \text { for all } l \in \mathcal{I}, \\
p_{l}^{*} C_{l}\left(\boldsymbol{\kappa}^{*}\right) & =0, \text { for all } l \in \mathcal{E} \cup \mathcal{I},
\end{aligned}
$$

where $\nabla_{\kappa}=\partial / \partial \kappa$ and the superimposed ${ }^{*}$ denotes a quantity evaluated at the solution. The conditions in Eq. (100) are known as the Karush-Kuhn-Tucker (KKT) conditions (Karush, 2014, Kuhn and Tucker, 2014). By analogy with the unconstrained case, if the Hessian of the Lagrangian is positive definite at a point fulfilling the first-order necessary conditions, that point is a strict minimizer of the constrained minimization problem (second-order sufficient condition).

Many different deterministic optimization algorithms have been developed to identify stationary points for unconstrained and constrained optimization problems. Algorithms based on the interior-point method have been found to efficiently solve the minimization problems considered in this dissertation. The algorithmic functionality, however, exceeds the scope of this section and the interested reader is referred to Nocedal and Wright (1999) for general information about interior-point methods and the manuals of the particular algorithms used in this dissertation: the interior-point algorithm of the MATLAB function fmincon (MATLAB, 2020); and the Interior Point Optimizer (IPOPT) (Wächter and Biegler, 2006).

In order to highlight a drawback of deterministic optimization algorithms, the calculation of the search direction for an unconstrained minimization problem is considered ${ }^{5}$. The modified Newton method is frequently used and the search direction $\boldsymbol{d}$ is computed according to (Nocedal and Wright, 1999)

$$
\boldsymbol{d}=-\underline{B}^{-1} \nabla_{\kappa} \varepsilon,
$$

where $\underline{B}$ is a positive definite approximation of the Hessian of the objective function. If the Hessian is easily calculated analytically, a favorable choice for $\underline{B}$ is

$$
\underline{B}=\nabla_{\kappa}^{2} \varepsilon+\psi \underline{I},
$$

where $\nabla_{\kappa}^{2} \varepsilon$ is the Hessian of the objective function, $\psi=\max \left(0,-\mu_{\min }\right), \mu_{\min }$ is the smallest eigenvalue of the Hessian, and $\underline{I}$ is the identity matrix (Nocedal and Wright, 1999). Otherwise $\underline{B}$ must be approximated completely, e.g. by the BFGS method (Fletcher, 2000).

\footnotetext{
${ }^{4}$ Fox example, Slater's constraint qualification, which states that the feasible region must have an interior point, must hold if only box constraints are considered. More constraint qualifications are found in Nocedal and Wright (1999).

${ }^{5}$ For a constrained minimization problem the system of equations becomes more complicated but is essentially the same.
} 


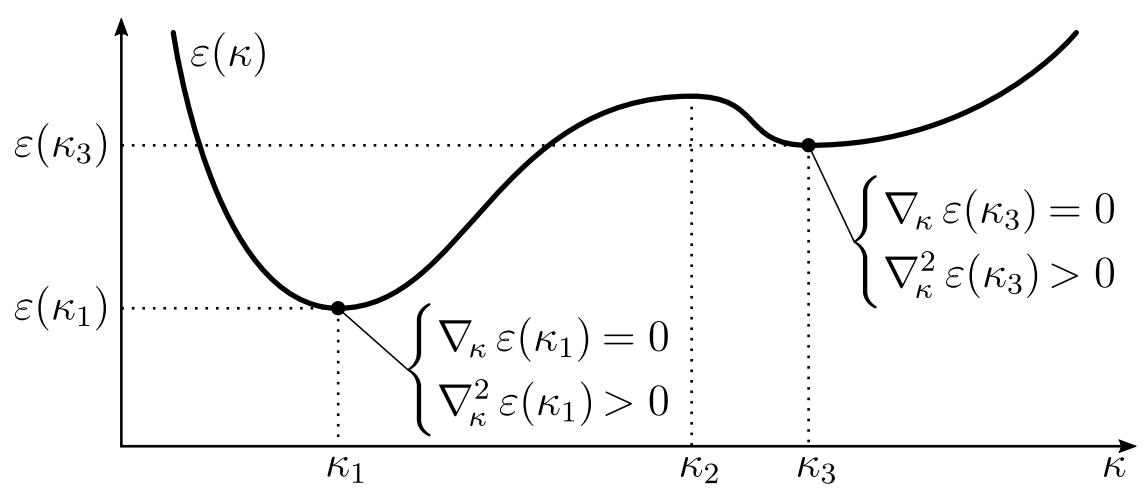

Figure 15: Simple one-dimensional objective function $\varepsilon(\kappa)$ plotted versus its argument $\kappa$. The global minimum of the objective function is located at $\kappa_{1}$. For starting points $\kappa>\kappa_{2}$ the local minimum at $\kappa_{3}$ is obtained if a gradient-based method is used.

The search directions defined in Eqs. (101) and (102) are descent directions and obviously an optimization algorithm stops once a stationary point has been identified since the search direction becomes the zero vector, cf. Eq. (101). They are, however, only local decent directions and even if the identified stationary point fulfills the second-order sufficient condition and is therefore a strict minimizer of the minimization problem, the found solution is only a strict minimizer locally. In case the minimization problem is non-convex, i.e. several stationary points exist, it is in practice not possible to determine whether the found local solution is the absolute minimum of the objective function, i.e. the global solution. This fundamental drawback is illustrated in Figure 15 for a simple one-dimensional objective function. For starting values of the model parameter $\kappa>\kappa_{2}$ not the global minimum located at $\kappa_{1}$, but the local minimum at $\kappa_{3}$ is obtained.

An obvious approach to increase the likelihood that the global minimum is identified is to initiate the optimization algorithm from multiple starting points and take the found local solution with the lowest objective function value as the bestguess for the global solution. Typically the starting points are randomly selected within the parameter space spanned by the box constraints on the model parameters, i.e. Monte Carlo Sampling. It is, however, computationally expensive to start from multiple starting points and to reduce the number while still representing the whole parameter space, more sophisticated sampling methods can be used. One such method is Latin Hypercube Sampling (McKay et al., 2000).

In Latin Hypercube Sampling the whole parameter space is divided into equally sized subspaces in which the starting points are selected. The difference between Monte Carlo Sampling and Latin Hypercube Sampling is illustrated for the selection of ten pairs of model parameters denoted $k_{1}, k_{2} \in[0,100]$ in Figure 16. As can be seen, the starting points are more evenly distributed in the parameter space using Latin Hypercube Sampling than with Monte Carlo Sampling. 


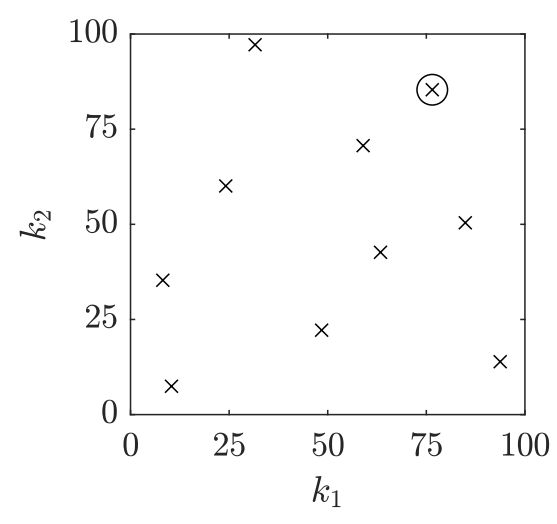

(a) Latin Hypercube Sampling

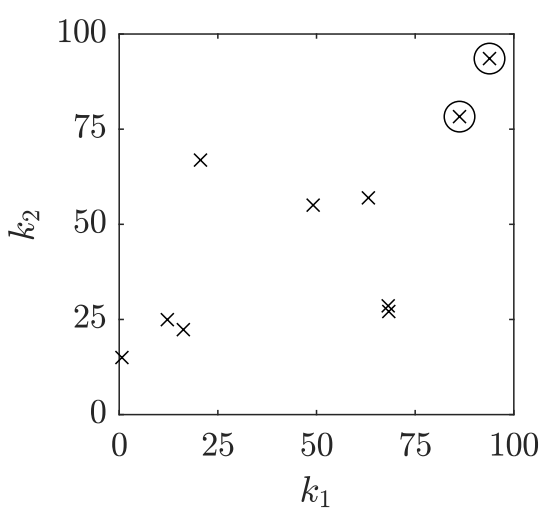

(b) Monte Carlo Sampling

Figure 16: Selection of ten starting points for parameters $k_{1}, k_{2} \in[0,100]$ using Latin Hypercube Sampling and Monte Carlo Sampling. A starting point is denoted by a diagonal cross and the circled starting points are assumed to produce an unrealistic model response.

To enhance the selection of starting points even further, it can be advantageous to set requirements for a starting point, e.g. that the starting point is feasible and that the predicted model response is somewhat reasonable. Assume therefore that starting points close to all upper bounds on the parameters, i.e. the circled starting points in the upper right corner of Figure 16, result in a completely unrealistic model response. If the model does not produce realistic results in this part of the parameter space, it is unlikely that the global solution is located there and it is more efficient to initiate the time consuming optimization algorithm from somewhere else. Since it is generally not known a priori which part of the solution space produces realisitic results, the number of starting points seeded using Latin Hypercube Sampling should be continuously increased until at least a certain amount of them fulfill the initially set requirements. Obviously this approach is only practicable if the time it takes to select advantageous starting points is compensated by the time spent solving the minimization problem and ultimately if the global solution (or a good estimate of it) is obtained.

Another aspect which can facilitate the numerical solving of the minimization problem is scaling. A problem is called poorly scaled if changes in one parameter cause a much larger response in the objective function compared to the other parameters. The contour lines of the objective function of a poorly scaled problem appear, therefore, as an ellipse, i.e. they are elongated, while they look like a circle for a well scaled problem. Although optimization algorithms using Newton's method to compute the search direction are in principle unaffected by a poorly scaled problem, it is still advisable to rescale a problem considering these guidelines (Betts, 2010, Nocedal and Wright, 1999): 
- Normalize the independent model parameters to have the same range.

- Normalize the objective and constraint functions to have the same magnitude.

- Scale the problem such that the condition number of the Hessian is close to one, i.e. that the minimum and maximum eigenvalue of the Hessian have approximately the same magnitude.

Despite a sophisticated heuristic method no guarantee can be given that indeed the global solution will be obtained. Deterministic global optimization methods on the other hand, guarantee that in finite time, a solution is found whose objective function value is at worst a user-specified value higher than that of the global solution (Horst and Tuy, 1996). In the next section, the Branch-and-Bound (B\&B) method is introduced which is used to construct a deterministic global optimization algorithm in Paper III.

\subsection{Branch-and-Bound method}

The basic idea of the $\mathrm{B} \& \mathrm{~B}$ method is to successively partition the parameter space, denoted $\Pi$, and exclude regions which cannot contain the global solution. For each partitioned parameter region two minimization problems are solved: an upper problem $(\mathbb{U P})$ providing an upper bound on the (globally) optimal value; and a lower problem $(\mathbb{L P})$ providing a lower bound on the optimal value. The original non-convex minimization problem can be taken for $(\mathbb{U P})$, while $(\mathbb{L} \mathbb{P})$ must be a convex relaxation of $(\mathbb{U P})$ with the requirement that $(\mathbb{L P})$ generates successively tighter lower bounds ${ }^{6}$ on the optimal value as the parameter space gets partitioned. During the process the $\mathrm{B} \& \mathrm{~B}$ method keeps track of the lowest upper bound found so far, denoted $U B$, and a list of active regions $\mathcal{A}$ containing subsets $\Pi_{i} \subseteq \Pi$ which potentially contain the global solution. For each $\Pi_{i}$ the corresponding lower bound $L B_{i}$ on the optimal value is stored in $\mathcal{A}$ as well. During each $\mathrm{B} \& \mathrm{~B}$ iteration, the subset $\Pi_{i}$ with the lowest lower bound $L B_{i}$ of all active regions is removed from $\mathcal{A}$ and partitioned into the new subsets $\varpi_{1}$ and $\varpi_{2}$, i.e. $\Pi_{i}=\varpi_{1} \cup \varpi_{2}$. This partitioning is called the branching step. Afterwards the, so called, bounding step is performed on the new subsets:

1. Solve $(\mathbb{U P})$ in $\varpi_{l}$ and if the upper bound, $U B_{l}$, fulfills $U B_{l}<U B$, set $U B=U B_{l}$.

2. Solve $(\mathbb{L} \mathbb{P})$ in $\varpi_{l}$ and if the lower bound, $L B_{l}$, fulfills $L B_{l}<U B$, add $\varpi_{l}$ and $L B_{l}$ to $\mathcal{A}$.

The bounding step causes, therefore, that the sequence of upper bounds $U B$ is non-increasing and that regions which cannot contain the global solution, because the lower bound on the optimal value in the region is higher than the lowest upper bound found so far, are discarded. Furthermore, as $(\mathbb{L P})$ generates successively tighter lower bounds on the optimal value, the lower bound $L B_{l}$ of the partitioned

\footnotetext{
${ }^{6}$ Tightness in the context of convex relaxation means that the difference between the non-convex function and its convex relaxation is small.
} 
(a) B\&B initialization

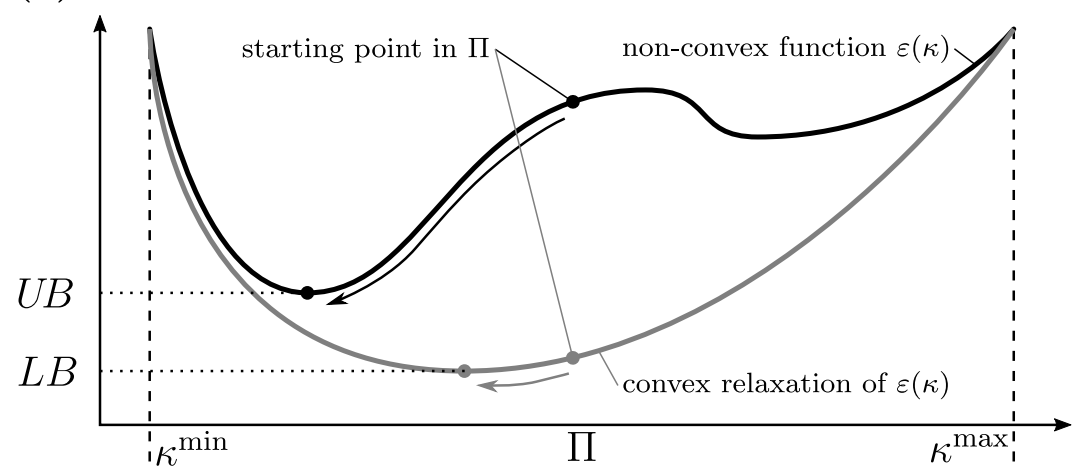

(b) $\mathrm{B} \& \mathrm{~B}$ iteration 1

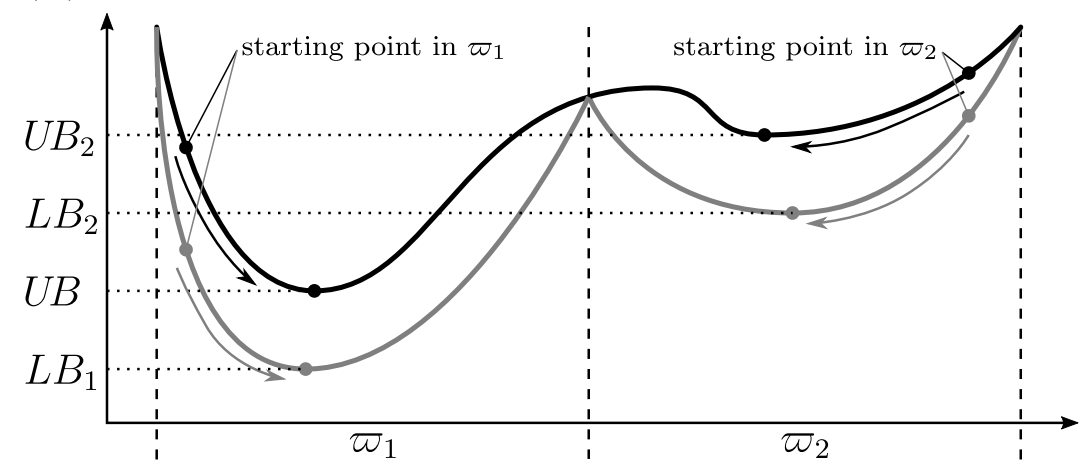

Figure 17: Initialization (a) and first iteration (b) of a B\&B algorithm applied to the one-dimensional objective function $\varepsilon(\kappa)$. The black line denotes $\varepsilon(\kappa)$ and the grey line its convex relaxation in the respective parameter range. By chance the global solution is identified during the initialization of the $\mathrm{B} \& \mathrm{~B}$ algorithm and $\Pi$ together with $L B$ are added to the list of active regions $\mathcal{A}$. During the first iteration $\Pi$ together with $L B$ are removed from $\mathcal{A}$ and since $L B$ and $U B$ are far apart, $\Pi$ is bisected into $\varpi_{1}$ and $\varpi_{2}$. The same solution is found for the upper problem in $\varpi_{1}$ and $U B$ does not need to be updated. Since $L B_{1}<U B$, the region $\varpi_{1}$ together with $L B_{1}$ are added to $\mathcal{A}$. In region $\varpi_{2}$, the upper bound is $U B_{2}>U B$ and $U B$ is not updated. Furthermore, the lower bound is $L B_{2}>U B$ and, therefore, the region $\varpi_{2}$ is discarded because it cannot contain the global solution. 
subset $\varpi_{l}$ fulfills $L B_{l} \geq L B_{i}$. Hence, the sequence of lower bounds $L B_{i}$ is nondecreasing and will converge towards the non-increasing sequence of upper bounds $U B$.

The $\mathrm{B} \& \mathrm{~B}$ algorithm is stopped once the relative difference of $L B_{i}$ and $U B$ is less than a user-specified tolerance $\tau$. In this context the terminology $\tau$-optimality ${ }^{7}$ is used and means that the relative difference of the found (local) optimal value and the globally optimal value is at worst $\tau$.

A particular algorithm based on this B\&B method is given in Paper III. In Figure 17 the initialization and the first iteration of a B\&B based algorithm are visualized for the simple one-dimensional objective function in Figure 15.

For the efficiency of a $B \& B$ algorithm the construction of $(\mathbb{L} \mathbb{P})$, i.e. the convex relaxation of $(\mathbb{U P})$, and the partitioning of the parameter space during the branching step are crucial. These two topics are covered in Sections 5.3.1 and 5.3.2 based on the following example of a non-convex minimization (upper example) problem:

$(\mathbb{U E P}) \begin{cases}\underset{\boldsymbol{\kappa} \in \mathbb{R}^{2+n}}{\operatorname{minimize}} & \sum_{j=1}^{n}\left[r_{\mathrm{i}, j}-r_{\mathrm{i}}^{\bmod }\left(\boldsymbol{\kappa}, P_{j}, h_{j}\right)\right]^{2}, \\ \text { subject to: } & \boldsymbol{\kappa}^{\min } \leq \boldsymbol{\kappa} \leq \boldsymbol{\kappa}^{\max },\end{cases}$

where $\boldsymbol{\kappa}=\left(c, \tilde{\lambda}_{\theta, j}, \tilde{\lambda}_{z}\right)$ and

$$
r_{\mathrm{i}, j}^{\bmod }=\frac{h_{j}}{P_{j}} c\left[\tilde{\lambda}_{\theta, j}-\left(\tilde{\lambda}_{\theta, j} \tilde{\lambda}_{z}\right)^{-1}\right] .
$$

Thus, for every $j=1, \ldots, n$ inner radius measurement, one model parameter $\tilde{\lambda}_{\theta, j}$ is identified. In order to shorten the notation, $\tilde{\lambda}_{\theta, j}$ instead of $\tilde{\lambda}_{\theta, 1}, \ldots, \tilde{\lambda}_{\theta, n}$ is used in the parameter vector $\boldsymbol{\kappa}$ and this abbreviating notation is used throughout.

The problem $(\mathbb{U E P})$ corresponds to fitting a thin-walled arterial model, which treats arterial tissue as an incompressible neo-Hookean material, to pressure-radius data, cf. Eq. (76) with $\alpha=0$ and the isotropic part of Eq. (83). Observe that $\tilde{\lambda}_{\theta, j}=\lambda_{\theta, m, j}^{2}$ and $\tilde{\lambda}_{z}=\lambda^{2}$ are used.

\subsubsection{Construction of convex relaxation}

The basic concept of constructing a convex relaxation of a non-convex function is to continuously reduce the degree of non-convexity by replacing non-convex terms by auxiliary variables in combination with constraints. Ready-to-use convex relaxations have been developed for a variety of non-linear functions, e.g. for bilinear, fractional, componentwise convex, and generally non-linear terms (Al-Khayyal, 1990, Maranas and Floudas, 1994, 1995, McCormick, 1976, Najman et al., 2019, Zamora and Grossmann, 1999) (Paper III). Multiple convex relaxations are possible for a nonconvex function and these variants will differ in tightness and required additional auxiliary variables and constraints.

\footnotetext{
${ }^{7}$ More common is to denote the tolerance $\varepsilon$ and therefore use the terminology $\varepsilon$-optimality. Since $\varepsilon$ is used for the objective function in this dissertation, the tolerance is denoted $\tau$.
} 
The problem $(\mathbb{U E P})$ is non-convex due to its non-linear model equation in Eq. (103) and the degree of non-linearity is amplified by the squaring in the leastsquares objective function. The amplification can be resolved by introducing the model equation not in the objective function directly but as an equality constraint instead (Esposito and Floudas, 1998). Auxiliary variables $w_{1, j}$ replacing the model equation are introduced and the intermediate example problem becomes

$(\mathbb{I E P}) \begin{cases}\underset{(\boldsymbol{\kappa}, \boldsymbol{w}) \in \mathbb{R}^{2+2 n}}{\operatorname{minimize}} & \sum_{j=1}^{n}\left[r_{\mathrm{i}, j}-w_{1, j}\right]^{2}, \\ \text { subject to: } \quad & \left\{\begin{array}{l}-w_{1, j}+\frac{h_{j}}{P_{j}} c\left[\tilde{\lambda}_{\theta, j}-\left(\tilde{\lambda}_{\theta, j} \tilde{\lambda}_{z}\right)^{-1}\right]=0, \\ \boldsymbol{w}^{\min } \leq \boldsymbol{w} \leq \boldsymbol{w}^{\max }, \\ \boldsymbol{\kappa}^{\min } \leq \boldsymbol{\kappa} \leq \boldsymbol{\kappa}^{\max },\end{array}\right.\end{cases}$

where $\boldsymbol{w}$ contains all auxiliary variables, and $\boldsymbol{w}^{\min }$ and $\boldsymbol{w}^{\max }$ are the lower and upper bound of $\boldsymbol{w}$, respectively. The objective function of $(\mathbb{E} \mathbb{E})$ is now convex in the auxiliary variable $w_{1, j}$ at the expense of introducing $n$ non-linear equality constraints. The non-linearity in the equality constraints is due to the terms $c \tilde{\lambda}_{\theta, j}$ and $c\left(\tilde{\lambda}_{\theta, j} \tilde{\lambda}_{z}\right)^{-1}$. Observe that the measurements $h_{j}$ and $P_{j}$ do not contribute to the degree of non-linearity since they act as simple factors.

The bilinear terms $c \tilde{\lambda}_{\theta, j}$ in $(\mathbb{I E P})$ are replaced by the auxiliary variables $w_{2, j}$ and $n$ times the following four linear inequality constraints are added:

$$
\begin{array}{r}
c \tilde{\lambda}_{\theta, j}^{\min }+\tilde{\lambda}_{\theta, j} c^{\min }-c^{\min } \tilde{\lambda}_{\theta, j}^{\min }-w_{2, j} \leq 0, \\
c \tilde{\lambda}_{\theta, j}^{\max }+\tilde{\lambda}_{\theta, j} c^{\max }-c^{\max } \tilde{\lambda}_{\theta, j}^{\max }-w_{2, j} \leq 0, \\
-c \tilde{\lambda}_{\theta, j}^{\min }-\tilde{\lambda}_{\theta, j} c^{\max }+c^{\max } \tilde{\lambda}_{\theta, j}^{\min }+w_{2, j} \leq 0, \\
-c \tilde{\lambda}_{\theta, j}^{\max }-\tilde{\lambda}_{\theta, j} c^{\min }+c^{\min } \tilde{\lambda}_{\theta, j}^{\max }+w_{2, j} \leq 0 .
\end{array}
$$

The first two linear inequality constraints in Eq. (104) represent the convex hull (McCormick, 1976), i.e. they bound $w_{2, j}$ from below, and the latter two the concave hull (Al-Khayyal, 1990), i.e. they bound $w_{2, j}$ from above.

Similarly, the terms $c\left(\tilde{\lambda}_{\theta, j} \tilde{\lambda}_{z}\right)^{-1}$ are relaxed, but three choices are possible:

(i) Relax the bilinear terms $\tilde{\lambda}_{\theta, j} \tilde{\lambda}_{z}$ first by replacing them by the auxiliary variables $w_{3, j}$ and afterwards relax the fractional terms $c w_{3, j}^{-1}$ by replacing them by $w_{4, j}$.

(ii) Relax the fractional terms $c \tilde{\lambda}_{\theta, j}^{-1}$ first by replacing them by the auxiliary variables $w_{3, j}$ and afterwards relax the fractional terms $w_{3, j} \tilde{\lambda}_{z}^{-1}$ by replacing them by $w_{4, j}$.

(iii) Relax the fractional term $c \tilde{\lambda}_{z}^{-1}$ first by replacing it by the auxiliary variable $w_{3}$ and afterwards relax the fractional terms $w_{3} \tilde{\lambda}_{\theta, j}^{-1}$ by replacing them by $w_{4, j}$.

Out of these three options, option (iii) introduces the lowest amount of additional auxiliary variables and is therefore preferable, cf. the missing index $j$ for $w_{3}$ in (iii). 
The four inequality constraints per fractional term are for $w_{3}$ :

$$
\begin{array}{r}
\frac{c}{\tilde{\lambda}_{z}^{\max }}+\frac{c^{\min }}{\tilde{\lambda}_{z}}-\frac{c^{\min }}{\tilde{\lambda}_{z}^{\max }}-w_{3} \leq 0, \\
\frac{c}{\tilde{\lambda}_{z}^{\min }}+\frac{c^{\max }}{\tilde{\lambda}_{z}}-\frac{c^{\max }}{\tilde{\lambda}_{z}^{\min }}-w_{3} \leq 0, \\
-\frac{c}{\tilde{\lambda}_{z}^{\min }}+\frac{\tilde{\lambda}_{z} c^{\min }}{\tilde{\lambda}_{z}^{\min } \tilde{\lambda}_{z}^{\max }}-\frac{c^{\min }}{\tilde{\lambda}_{z}^{\max }}+w_{3} \leq 0, \\
-\frac{c}{\tilde{\lambda}_{z}^{\max }}+\frac{\tilde{\lambda}_{z} c^{\max }}{\tilde{\lambda}_{z}^{\min } \tilde{\lambda}_{z}^{\max }}-\frac{c^{\max }}{\tilde{\lambda}_{z}^{\min }}+w_{3} \leq 0,
\end{array}
$$

and similarly for $w_{4, j}$. The first two inequality constraints in Eq. (105) are convex and represent the convex hull of $w_{4, j}$ (Maranas and Floudas, 1995). The latter two inequality constraints are linear and represent the concave hull (Zamora and Grossmann, 1999). At this point the non-convexity of $(\mathbb{U E P})$ has been taken care of and the relaxed (lower) example problem reads:

$(\mathbb{L} \mathbb{E} \mathbb{P}) \begin{cases}\underset{(\boldsymbol{\kappa}, \boldsymbol{w}) \in \mathbb{R}^{3+4 n}}{\operatorname{minimize}} & \sum_{j=1}^{n}\left[r_{\mathrm{i}, j}-w_{1, j}\right]^{2}, \\ \text { subject to: } \quad \begin{array}{l}-w_{1, j}+\frac{h_{j}}{P_{j}}\left(w_{2, j}-w_{4, j}\right)=0, \\ \text { constraints for bilinear terms: } w_{2, j} \rightarrow c \tilde{\lambda}_{\theta, j}, \\ \text { constraints for fractional term: } w_{3} \rightarrow c \tilde{\lambda}_{z}^{-1}, \\ \text { constraints for fractional terms: } w_{4, j} \rightarrow w_{3} \tilde{\lambda}_{\theta, j}^{-1}, \\ \boldsymbol{w}^{\text {min }} \leq \boldsymbol{w} \leq \boldsymbol{w}^{\text {max }}, \\ \boldsymbol{\kappa}^{\text {min }} \leq \boldsymbol{\kappa} \leq \boldsymbol{\kappa}^{\text {max }},\end{array}\end{cases}$

where the notation $\rightarrow$ denotes an auxiliary variable replacing the non-linear term following the arrow. The convex problem $(\mathbb{L} \mathbb{E P})$ has in total $3+4 n$ unknown variables $(2+n$ model parameters and $1+3 n$ auxiliary variables $), n$ linear equality constraints, $2+6 n$ linear inequality constraints, $2+2 n$ convex inequality constraints, and $6+8 n$ simple box constraints. The original non-convex problem $(\mathbb{U E P})$ has in comparison only $2+n$ unknown variables and $4+2 n$ simple box constraints.

Figure 18 visualizes the original non-linear model Eq. (103) and its convex relaxation used in $(\mathbb{L E P})$. The thick line represents the non-linear model Eq. (103) as a function of $\tilde{\lambda}_{\theta, j}$ with $h_{j}=P_{j}=c=\tilde{\lambda}_{z}=1$. In $(\mathbb{L} \mathbb{E} \mathbb{P})$ this model equation is replaced by the auxiliary variable $w_{1, j}$. The remaining auxiliary variables together with the constraints form a convex set for $w_{1, j}$ which is indicated by the grey area in between the convex and concave hull of $w_{1, j}$. Hence, while in $(\mathbb{U E P})$ the optimization algorithm has to select $r_{\mathrm{i}, j}$ on the hypersurface represented by the solid black line, the algorithm can chose $w_{1, j}$ freely from the grey area in $(\mathbb{L} \mathbb{E} \mathbb{P})$. The smaller the convex set is for an auxiliary variable the tighter is the convex relaxation and the faster a $\mathrm{B} \& \mathrm{~B}$ algorithm will certify $\tau$-optimality. 


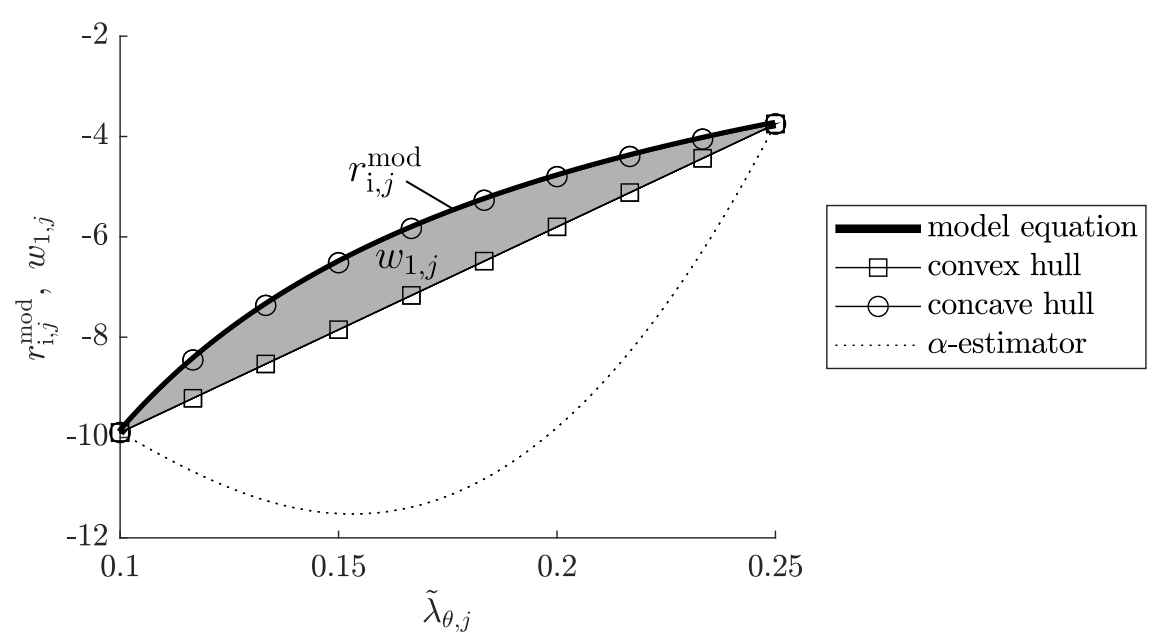

Figure 18: Original non-linear model Eq. (103) and its convex relaxation used in $(\mathbb{L} \mathbb{E} \mathbb{P})$. The thick line denotes the model equation, and the lines with circles and squares denote the concave and convex hull of the model equation, respectively. The grey area represents the convex set of the auxiliary variable $w_{1, j}$. The dotted line represents an alternative convex hull of the model equation determined with the $\alpha$-estimator.

To highlight the efficiency of the presented convex relaxation, the convex hull obtained by using the $\alpha$-estimator (Maranas and Floudas, 1994) to underestimate the non-linear model equation is also included in Figure 18. As can be seen, $(\mathbb{L} \mathbb{E} \mathbb{P})$ appears to be the tightest possible convex relaxation of $(\mathbb{U E P})$.

\subsubsection{Branching method}

During the branching step a decision must be made on how the current parameter space should be partitioned, cf. $\Pi_{i}$ in Section 5.3. The simplest form of partitioning is to select a parameter and bisect its range. Both the model parameters and the auxiliary variables can be partitioned. Afterwards each new parameter space must be determined by computing the lower and upper bounds on all parameters and auxiliary variables. The calculation of the new parameter space is trivial if a model parameter is bisected but becomes complicated if an auxiliary variable is bisected instead. Consider therefore the auxiliary variable $w_{2, j}$ replacing the bilinear term $c \tilde{\lambda}_{\theta, j}$, cf. Section 5.3.1. The lower and upper bound of $w_{2, j}$ in a given region where $c \in\left[c^{\min }, c^{\max }\right]$ and $\tilde{\lambda}_{\theta, j} \in\left[\tilde{\lambda}_{\theta, j}^{\min }, \tilde{\lambda}_{\theta, j}^{\max }\right]$ are easily calculated:

$$
w_{2, j}^{\min }=c^{\min } \tilde{\lambda}_{\theta, j}^{\min } \quad \text { and } \quad w_{2, j}^{\max }=c^{\max } \tilde{\lambda}_{\theta, j}^{\max }
$$

Hence, if the model parameters $c$ or $\tilde{\lambda}_{\theta, j}$ are bisected, the bounds in Eq. (106) are easily updated. If instead $w_{2, j}$ is bisected, it is not trivial how the bounds 
on $c, \tilde{\lambda}_{\theta, j}$, and all other auxiliary variables are affected. In Esposito and Floudas (1998) it is suggested to determine those bounds by an additional optimization step. The additional optimization step, which has to be performed twice during every $\mathrm{B} \& \mathrm{~B}$ iteration, becomes very time consuming and only branching on the model parameters is therefore considered in this dissertation.

The selection of a parameter to bisect should be driven by the goal to tighten the convex relaxation as much as possible. Several strategies have been proposed:

Strategy 1: Bisect the model parameter which has been bisected the fewest times, i.e. the one which has the largest region reduction measure defined as

$$
r_{x}=\frac{x^{\max }-x^{\min }}{x^{\text {max }, \text { orig }}-x^{\text {min,orig }}},
$$

where $x$ is any model parameter and the superscript orig denotes original (Adjiman et al., 1998). Hence, in Eq. (107) the ratio of the parameter range in the current subspace is divided by the parameter range in the original parameter space.

Strategy 2: Determine the auxiliary variable which deviates the most from the term it replaces at the solution of the lower bounding problem. Bisect the model parameter involved in this auxiliary variable which has the largest reduction measure according to Strategy 1 (Adjiman et al., 1998). As an example, the deviation for the auxiliary variable $w_{2, j}$ replacing the bilinear term $c \tilde{\lambda}_{\theta, j}$ is given by

$$
\delta_{2, j}=\left|c^{*} \tilde{\lambda}_{\theta, j}^{*}-w_{2, j}^{*}\right|,
$$

where $c^{*}, \tilde{\lambda}_{\theta, j}^{*}$, and $w_{2, j}^{*}$ are the values at the solution of $(\mathbb{L} \mathbb{E} \mathbb{P})$.

Strategy 3: Determine the model parameter which influences all auxiliary variables the most by adding all deviations determined in Strategy 2 that the model parameter is involved in (Adjiman et al., 1998). As an example, the total deviation for the model parameter $c$ in $(\mathbb{L} \mathbb{E P})$ is:

$$
\delta_{c}=\left|\frac{c^{*}}{\tilde{\lambda}_{z}^{*}}-w_{3}^{*}\right|+\sum_{j=1}^{n}\left|c^{*} \tilde{\lambda}_{\theta, j}^{*}-w_{2, j}^{*}\right| .
$$

Strategy 4: Determine the product of region reduction measure according to Strategy 1 and total deviation according to Strategy 3 for every model parameter (Paper III). Bisect the model parameter with the highest product. As an example, the product for the model parameter $c$ in $(\mathbb{L E P})$ is:

$$
\delta_{c}=\frac{c^{\max }-c^{\min }}{c^{\text {max }, \text { orig }}-c^{\text {min,orig }}}\left(\left|\frac{c^{*}}{\tilde{\lambda}_{z}^{*}}-w_{3}^{*}\right|+\sum_{j=1}^{n}\left|c^{*} \tilde{\lambda}_{\theta, j}^{*}-w_{2, j}^{*}\right|\right) .
$$

Additional, although similar, branching strategies can be found in Adjiman et al. (1998), Esposito and Floudas (1998). 



\section{In Vivo Parameter Identification Method}

The in vivo parameter identification method determines the mechanical properties of an artery by fitting a non-linear continuum-mechanical model, the constitutive membrane model in Section 4.2, to clinical measurements. The required clinical measurements are either triplets of blood pressure, radial deformation of the arterial wall, and wall thickness or alternatively pairs of blood pressure and radial deformation of the arterial wall, and information about the current cross-sectional area for one pressure-radius pair. Both types of raw data sets are pre-processed and converted into a, so called, pressure-radius data set containing the current cross-sectional area $A$ and $n$ triplets of blood pressure $P$, inner radius $r_{\mathrm{i}}$, and wall thickness $h$. The pre-processing routine is described in Section 6.1.

Once the clinical measurements have been pre-processed, the model parameters are tuned such that the constitutive membrane model is able to explain the pressureradius data set, and the resulting best-fit model parameters are the mechanical properties of the artery in question. The tuning is formulated in terms of a weighted least-squares minimization problem. Depending on whether smooth muscle activity is accounted for or neglected, the minimization problem is slightly different. In the following, the passive constitutive membrane model is used and its active extension is considered separately in Section 6.4.

The following objective function is used for the passive constitutive membrane model:

$$
\begin{array}{r}
\varepsilon^{\mathrm{pas}}(\boldsymbol{\kappa})=\sum_{j=1}^{n}\left\{w\left[\sigma_{\theta \theta}^{\mathrm{Lp}}\left(P_{j}, r_{\mathrm{i}, j}, h_{j}\right)-\sigma_{\theta \theta}^{\mathrm{pas}}\left(\boldsymbol{\kappa}, r_{\mathrm{i}, j}, h_{j}\right)\right]^{2}+\ldots\right. \\
\left.(1-w)\left[\sigma_{z z}^{\mathrm{Lp}}\left(P_{j}, r_{\mathrm{i}, j}, h_{j}\right)-\sigma_{z z}^{\mathrm{pas}}\left(\boldsymbol{\kappa}, r_{\mathrm{i}, j}, h_{j}\right)\right]^{2}\right\},
\end{array}
$$

where $\boldsymbol{\kappa}=\left(R_{\mathrm{i}}, \lambda, c, k_{1}, k_{2}, \beta\right)$ is the parameter vector, $w \in[0,1]$ is a weighting factor to control the contribution of the circumferential and axial part, and $\sigma_{\theta \theta}^{\mathrm{Lp}}, \sigma_{z z}^{\mathrm{Lp}}$, $\sigma_{\theta \theta}^{\text {pas }}$, and $\sigma_{z z}^{\text {pas }}$ are given by Eqs. (76), (77), (83), and (84), respectively. Hence, the pressure-radius data set is converted into equilibrium stresses $\sigma_{\theta \theta}^{\mathrm{Lp}}$ and $\sigma_{z z}^{\mathrm{Lp}}$ to obtain an implicit normalization and a weighting factor is added, see Normalization 1 and 3 in Section 5.1. Observe that both the equilibrium and constitutively determined stresses are zero in the radial direction due to the thin-walled assumption and are therefore neglected in Eq. (111), cf. Section 4.2. Furthermore, the weighting factor is set to $w=0.99$ to let the objective function be dominated by the circumferential part while the axial part, which contains the estimated reduced axial force, is only 
Table 1: Box constraints on the model parameters used for the human abdominal aorta.

\begin{tabular}{ccccccc}
\hline Parameter & $\begin{array}{c}R_{\mathrm{i}} \\
{[\mathrm{mm}]}\end{array}$ & $\begin{array}{c}\lambda \\
{[-]}\end{array}$ & $\begin{array}{c}c \\
{[\mathrm{kPa}]}\end{array}$ & $\begin{array}{c}k_{1} \\
{[\mathrm{kPa}]}\end{array}$ & $\begin{array}{c}k_{2} \\
{[-]}\end{array}$ & $\begin{array}{c}\beta \\
{[\mathrm{deg}]}\end{array}$ \\
\hline $\boldsymbol{\kappa}^{\min }$ & 1 & 1 & $10^{-4}$ & $10^{-4}$ & $10^{-4}$ & 0 \\
$\boldsymbol{\kappa}^{\max }$ & 20 & 1.5 & $10^{3}$ & $10^{3}$ & $10^{3}$ & 90 \\
\hline
\end{tabular}

used as a regularization. The complete parameter identification problem reads:

$(\mathbb{P I}) \begin{cases}\underset{\kappa \in \mathbb{R}^{6}}{\operatorname{minimize}} & \varepsilon^{\text {pas }}(\boldsymbol{\kappa}), \\ \text { subject to: } & \boldsymbol{\kappa}^{\text {min }} \leq \boldsymbol{\kappa} \leq \boldsymbol{\kappa}^{\max } .\end{cases}$

The box constraints $\boldsymbol{\kappa}^{\min }$ and $\boldsymbol{\kappa}^{\max }$ are introduced to limit the parameter space but chosen wide enough such that they do not become active during the minimization. Furthermore, the lower bounds on the material parameters $c, k_{1}$, and $k_{2}$ are required to be positive to ensure that the strain-energy function is convex with respect to the deformation (Truesdell and Noll, 1992) and the angle $\beta$ is bounded by $\beta \in[0, \pi / 2]$ because it appears only in trigonometric functions. In order to prevent buckling in the axial direction, which the constitutive membrane model does not account for, the lower bound of the axial prestretch is set to $\lambda^{\mathrm{min}}=1$. The box constraints ${ }^{1}$ used for the human abdominal aorta are summarized in Table 1.

The minimization problem $(\mathbb{P I})$ is well-posed, smooth, but non-convex. The latter implies that it may contain several local minima that are not global minima (Nocedal and Wright, 1999). To address this issue, the heuristic method described in Section 5.2 is used. The gradient-based optimization algorithm is initiated from 100 starting points and the found local minimum with the lowest objective function value is taken to be the global minimum. The starting points are selected using Latin Hypercube Sampling and are required to produce stress values in the range $0 \mathrm{kPa} \leq \sigma_{\theta \theta}^{\bmod }, \sigma_{z z}^{\text {mod }} \leq 10^{5} \mathrm{kPa}$ which are loosely based on reported values for human arteries (Gasser et al., 2002, Holzapfel and Ogden, 2015, Labrosse et al., 2013). Furthermore, the objective function, gradient, and Hessian evaluated at a starting point are required to only contain real numbers.

In order to improve the scaling of the minimization problem, the model parameters $c, k_{1}, k_{2}$, and $\beta$ are replaced by scaled counterparts according to ${ }^{2}$

$$
c=\mathrm{e}^{\tilde{c}}, \quad k_{1}=\mathrm{e}^{\tilde{k}_{1}}, \quad k_{2}=\mathrm{e}^{\tilde{k}_{2}}, \quad \text { and } \quad \beta=\arcsin \sqrt{\tilde{\beta}} .
$$

The minimization problem $(\mathbb{P I})$ is solved in MatlaB (The MathWorks Inc., Natick, MA, USA). A MultiStart class is defined to initiate the interior-point optimization algorithm of the function fmincon from the generated 100 starting points. Analytical expressions for the gradient and Hessian of the objective function

\footnotetext{
${ }^{1}$ Inconsequentially wider box constraints are used in Paper I.

${ }^{2}$ In Paper I, $\beta=\arcsin \tilde{\beta}$ is used which results in a slightly poorer scaled problem.
} 
in Eq. (111) are determined with MAPLE (Maplesoft, Waterloo, Ontario) and provided to fmincon to facilitate the minimization.

After the description of the pre-processing routine in Section 6.1, the presented in vivo parameter identification method for arteries is validated for the human abdominal aorta in Section 6.2. In Section 6.3 the heuristic approach to solve the minimization problem $(\mathbb{P I})$ is replaced by a global optimization approach which ensures that a global minimum is obtained. Finally, the passive constitutive membrane model is extended to account for smooth muscle activity in Section 6.4.

\subsection{Pre-processing clinical data}

The clinical raw data consist of simultaneous measurements of blood pressure $\breve{P}$, radial deformation generally in terms of the inner radius $\breve{r}_{\mathrm{i}}$, and possibly thickness of the arterial wall $h$ typically in terms of the intima-media thickness (IMT). For the remainder it is assumed that the raw data contains $\breve{P}, \breve{r}_{\mathrm{i}}$, and $\breve{h}_{\mathrm{IMT}}$ measurements.

The raw data are pre-processed to generate a standardized pressure-radius data set consisting of current cross-sectional area $A$ of the arterial wall and $n$ triplets of blood pressure $P$, inner radius $r_{\mathrm{i}}$, and arterial wall thickness $h$. The pre-process follows the routine proposed in Stålhand (2009):

Step 1: Remove disturbances resulting from noise in the measurements, respiratory artifacts, and measurement errors, e.g. by involuntary movement of the measurement probe.

Step 2: Re-align the measurements in time to remove an offset which is possibly introduced through the measurement setup.

Step 3: Segment the measurements into consecutive pulses associated with the cardiac cycles.

Step 4: Calculate an average pulse using the segmented pulses.

Step 5: Re-sample the average pulse.

First of all the raw data are visually inspected and reduced to the part which contain somewhat stable $\breve{P}$ and $\breve{r}_{\mathrm{i}}$ measurements. The $\breve{h}_{\mathrm{IMT}}$ measurement is reduced to the same part but is not considered during the decision. Afterwards, the measurements are zero-phase filtered through a low-pass fourth-order Butterworth filter with cut-off frequency $15 \mathrm{~Hz}$ (Stålhand, 2009).

In step 2 , the time delay between the $\breve{P}$ and $\breve{r}_{\mathrm{i}}$ measurements is estimated and all three measurements are re-aligned following the routine described in Stålhand (2009). Furthermore, the cross-sectional area of the arterial wall $A$ is calculated using

$$
A=\operatorname{average}\left(2 \pi \breve{r}_{\mathrm{i}} \Upsilon \breve{h}_{\mathrm{IMT}}+\pi\left[\Upsilon \breve{h}_{\mathrm{IMT}}\right]^{2}\right),
$$



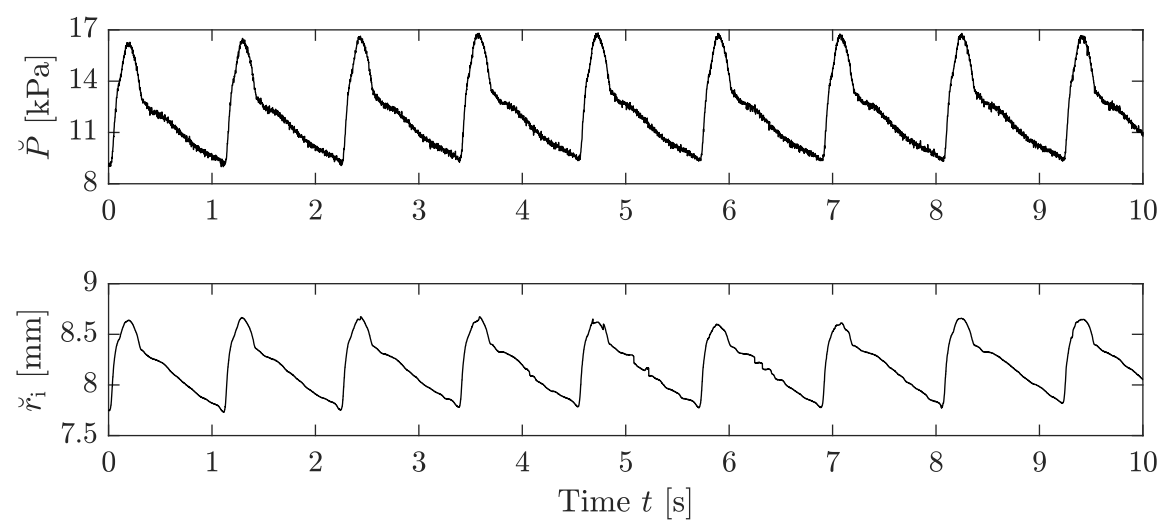

(a) Raw data

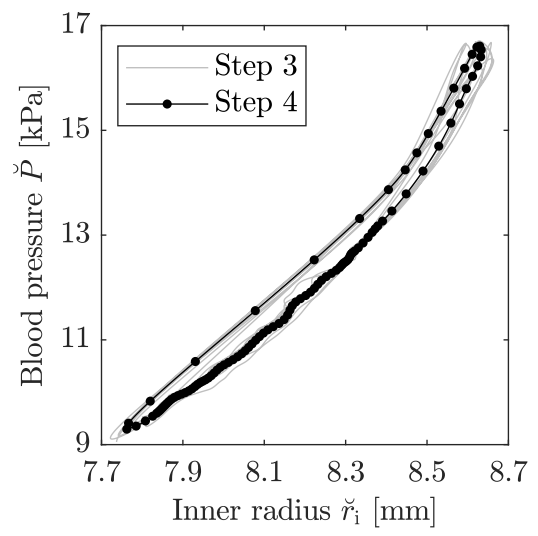

(b) Step 3 and 4

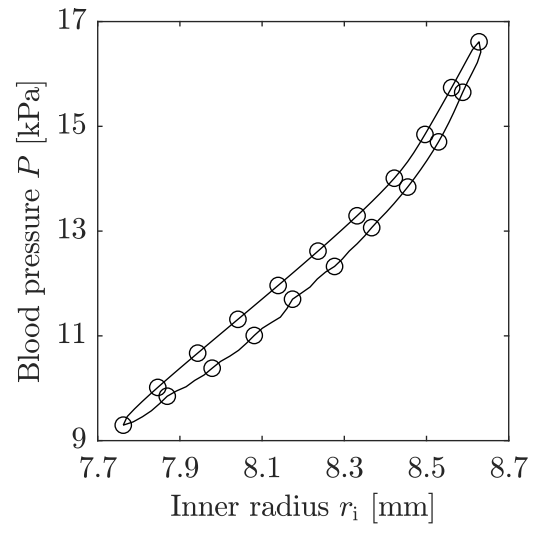

(c) Step 5

Figure 19: Pre-processing the raw data of the abdominal aorta in a healthy 24 -year-old male. In (a) the raw blood pressure and inner radius measurements are presented. Only measurement noise is visual and the complete raw data are used. The grey lines in (b) denote the data obtained after step 3, i.e. once the raw data in (a) are segmented into consecutive cycles, and the black line represents the average pressure-radius loop after step 4. Furthermore, every tenth sample of the average loop is indicated by a black dot, thus indicating that far more samples are collected during diastole compared to systole. In (c) the average loop is re-sampled using $n=20$ path equidistant samples denoted by a circle, i.e. step 5 . Data is taken from Sonesson et al. (1994). 
where $\Upsilon$ is a factor converting $\breve{h}_{\text {IMT }}$ into the arterial wall thickness $\breve{h}$, i.e. the intima-media-adventitia thickness. For the human abdominal aorta this factor is $\Upsilon=3 / 2$ (Holzapfel et al., 2007). In case the wall thickness is not included in the clinical data, the cross-sectional area is estimated, e.g. by using population averaged data (Stålhand, 2009) (Paper IV).

The segmentation of the continuous-time measurements into consecutive cycles is done in step 3 based on the onset of the sharp increase of $\breve{P}$ during systole. The sharp increase is identified by evaluating the second time derivative of $\breve{P}$ which has a characteristic peak-up-peak-down pattern at the onset of systole (Stålhand, 2009). The second time derivative is estimated through the central difference scheme.

In step 4 , an average $\breve{P}-\breve{r}_{\mathrm{i}}$ loop is calculated from the consecutive pulse cycles using the, so called, corrected integral shape averaging (CISA) method (Boudaoud et al., 2007, Stålhand, 2009).

Lastly, the average $\breve{P}-\breve{r}_{\mathrm{i}}$ loop is split into a systolic and a diastolic flank, where both flanks contain the diastolic and systolic blood pressure. Each flank is normalized and re-sampled using $n / 2+1$ path equidistant samples. Afterwards both flanks are combined and the duplicate diastolic and systolic samples are removed. Hence, the loop contains $n$ samples and is denoted the $P-r_{\mathrm{i}}$ loop. The presented re-sampling ensures that both the low and high pressure domain but also the systolic and diastolic flank will be weighted equally in the parameter identification. To conclude step 5, the current cross-sectional wall thickness $h$ is computed using Eq. (53) with $r_{\mathrm{i}}$ and $A$ calculated in Eq. (113) as

$$
h=-r_{\mathrm{i}}+\sqrt{r_{\mathrm{i}}^{2}+\frac{A}{\pi}}
$$

The calculation of $h$ according to Eq. (114) ensures an incompressible deformation which the constitutive membrane model assumes.

Finally, the pressure-radius data set containing $n\left(P, r_{\mathrm{i}}, h\right)$ triplets and $A$ is assembled which concludes the pre-processing routine. In Figure 19 the pre-processing steps 3,4 , and 5 are visualized.

\subsection{Validation}

In order to check whether the proposed in vivo parameter identification method identifies the desired mechanical properties of an artery correctly it needs to be validated.

The preferred experimental environment for arteries are controlled in vitro tests. Depending on the testing set-up, axial loading, twisting moments and perivascular state can be controlled and measured. By placing the artery in a chemical solution the in vivo environment is reasonably well replicated and by performing a series of stress-relieving cuts the (presumably) stress-free reference configuration can be determined. For an overview of in vitro testing devices and protocols the reader is referred to Humphrey (2002) and references therein. Classically bi-axial extension or inflation tests are performed to determine the model parameters for a 
specific constitutive model. Despite the controlled experimental environment and the possibility to measure e.g. layer dependent collagen fiber orientation, the model parameters must be identified by solving a minimization problem similar to $(\mathbb{P I})$ and the same complications as discussed in Section 5.2 arise. Furthermore, in vitro experiments of vascular tissue are complicated, measurement inaccuracies arise, and when dealing with human tissue ethical approval is required.

An alternative to in vitro tests are computer-based, i.e. in silico, experiments using arterial-like finite element models. In contrast to the in vitro case, the finite element environment is completely controllable since all quantities are either specified or can be measured. This is especially useful when validating an in vivo parameter identification method because the mechanical properties of the artery in question are pre-defined in terms of the model parameters and it is therefore possible to validate the method, not only qualitatively but also quantitatively. In addition, certain arterial characteristics can be included or excluded in the finite element model to study its effect on the parameter identification method. Nevertheless, in silico experiments are only an intermediate step and ultimately in vitro tests should be performed to fully validate the in vivo parameter identification method.

In total, 22 in silico arteries are created using model parameters of the human abdominal aorta from the literature. These arteries are based on the general continuum-mechanical model in Section 4.1 and respect, therefore, the thick-walled nature of an artery, account for residual stress, and the axial prestretch is chosen such that the reduced axial force is approximately constant throughout the cardiac cycle. An in silico artery is, however, assumed to be a homogeneous cylinder consisting of an isotropic matrix with embedded collagen fibers and the active contribution of smooth muscle cells is neglected. The created in silico arteries consider therefore more arterial characteristics than the passive constitutive membrane model but the straightforward comparison is nevertheless possible. For more information about the in silico arteries the reader is referred to Papers I and II.

Nine out of the 22 in silico arteries possess a significantly lower value for the parameter $c(p$-value $<0.01)$ associated with the non-collagenous matrix material. Such low values for $c$ have only been reported for the elderly and for abdominal aortic aneurysms (Ferruzzi et al., 2011), and these nine sets are, therefore, referred to as pathological. The remaining 13 arteries are considered healthy.

With these in silico arteries, in vivo-like raw data are generated and the mechanical properties are identified using the proposed in vivo parameter identification method. Solving the minimization problem $(\mathbb{P I})$ with MATLAB version R2019b takes about one minute on a standard hexa-core computer and approximately $80 \%$ of all starting points converge to the same solution with the lowest error function value for the healthy arteries ${ }^{3}$. For the pathological arteries significantly fewer

\footnotetext{
${ }^{3}$ It is stated in Paper I that the average time to solve the minimization problem is 39 minutes and that only $59 \%$ of all starting points converge to the same solution with the lowest objective function value. The calculation of the gradient and Hessian was, however, erroneous for the case $I_{4}<1$, i.e. when the anisotropic part of the strain-energy function is neglected. Affected starting points were not able to converge and the optimization was terminated once the iteration limit of $10^{5}$ was reached. The best-fit model parameters fulfill $I_{4}>1$ and were successfully identified.
} 
Table 2: Mean difference and $95 \%$ limits of agreement for healthy arteries (Paper I).

\begin{tabular}{cccc}
\hline Parameter & Unit & Mean difference & $95 \%$ limits of agreement \\
\hline$R_{\mathrm{i}}$ & {$[\mathrm{mm}]$} & 0.09 & $-0.34-0.52$ \\
$\lambda$ & {$[-]$} & 0.04 & $-0.13-0.20$ \\
$c$ & {$[\mathrm{kPa}]$} & 0.36 & $-1.80-2.51$ \\
$k_{1}$ & {$[\mathrm{kPa}]$} & -0.01 & $-10.23-10.21$ \\
$k_{2}$ & {$[-]$} & $0.98^{\mathrm{a}}$ & $0.75-1.21^{\mathrm{a}}$ \\
$\beta$ & {$[\mathrm{deg}]$} & -3.80 & $-9.97-2.37$ \\
\hline
\end{tabular}

${ }^{a}$ Mean ratio and the corresponding $95 \%$ limits of agreement are presented instead.

starting points, on average $5 \%$, converge to the same solution $(p$-value $<0.01)$ and the Hessian is rank-deficient at the solution in three cases.

In the following subsections Sections 6.2.1 and 6.2.2 the identified model parameters and the predicted stress state are separately compared to the in silico arteries.

\subsubsection{Model parameters}

By comparing the identified model parameters with the pre-defined ones of the in silico arteries the in vivo parameter identification method can be quantitatively validated.

In case of healthy arteries, the method overestimates $R_{\mathrm{i}}, \lambda$, and $c$ on average and underestimates $k_{1}, k_{2}$, and $\beta$ on average, see Table 2 . For parameter $k_{2}$ the differences between identified and pre-defined values increase as the pre-defined values increase. To compensate for this systematic error, the ratio of identified and pre-defined values is presented instead. Table 2 also contains the $95 \%$ limits of agreement representing the range one would expect the difference between identified and correct parameter to lie in, in $95 \%$ of the cases. The smaller the range the better the in vivo parameter identification method is able to identify a certain parameter.

For arteries with a pathologically small isotropic matrix contribution, unphysiological parameters are identified. This circumstance together with fewer starting points converging to the same solution and the Hessian losing its rank at the solution reveals the presence of a pathological artery, however.

Hence, the comparison indicates that the proposed in vivo parameter identification method determines adequate parameters for healthy arteries and exposes pathological arteries from in vivo-like pressure-radius measurements.

For detailed information about the quantitative validation of the in vivo parameter identification method the reader is referred to Paper I. 


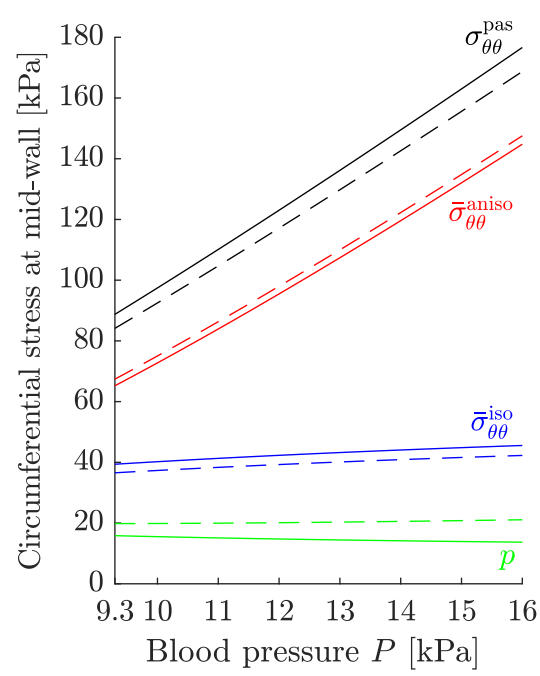

(a) Artery with small transmural stress gradient $\left(\left|\sigma_{\theta \theta}^{\text {pas }}\left(r_{\mathrm{i}}\right)-\sigma_{\theta \theta}^{\text {pas }}\left(r_{\mathrm{o}}\right)\right|<42 \mathrm{kPa}\right)$

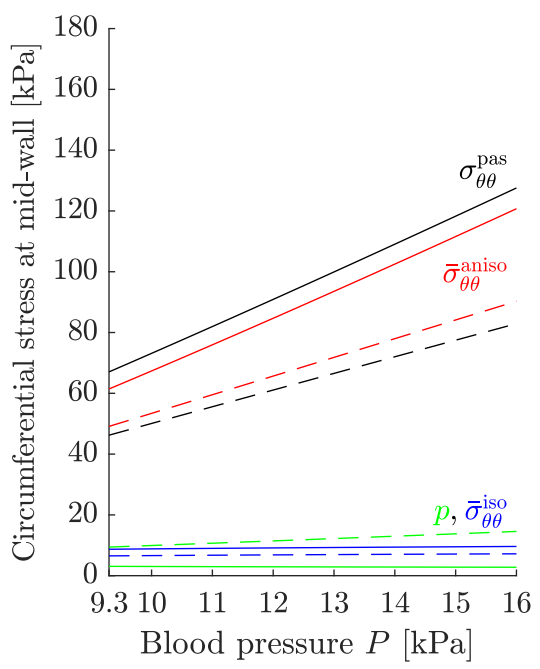

(b) Artery with large transmural stress gradient $\left(\left|\sigma_{\theta \theta}^{\text {pas }}\left(r_{\mathrm{i}}\right)-\sigma_{\theta \theta}^{\text {pas }}\left(r_{\mathrm{o}}\right)\right|>210 \mathrm{kPa}\right)$

Figure 20: Circumferential stress state during cardiac cycle for two representative arteries. The solid lines represent the predicted values of the passive constitutive membrane model using in vivo identified parameters. The dashed lines are associated with the stress state in the mid-wall of the in silico artery. The black color represents the total stress $\sigma_{\theta \theta}^{\text {pas }}$, red the anisotropic stress $\bar{\sigma}_{\theta \theta}^{\text {aniso }}$, blue the isotropic stress $\bar{\sigma}_{\theta \theta}^{\text {iso }}$ and green the reaction stress $p$. Data is taken from Paper II: (a) corresponds to set 15 (F63); and (b) to set 1 (F49).

\subsubsection{Stress state}

The identified model parameters are used in the passive constitutive membrane model to predict the stress state of the artery in question. The stress state is decomposed into a reaction stress, an isotropic stress, and an anisotropic stress which are associated with the incompressibilty constraint, the isotropic matrix, and the collagen fibers, respectively.

The agreement of the predicted decomposed stress state and the correct in silico stress state depends on the transmural stress gradient in the in silico arteries. For arteries experiencing a small transmural gradient, the decomposed stress prediction in the circumferential direction shows a high agreement with the in silico one throughout the cardiac cycle, see Figure 20a. The agreement deteriorates with increasing transmural gradient, see Figure 20b. Despite the identified unphysiological parameters for arteries with a pathologically small isotropic matrix contribution, the agreement of the predicted and the in silico stress states is similar to healthy arteries experiencing the same transmural stress gradient.

Larger differences between the predicted and the in silico stress state occur in 
the axial direction and is related to an erroneous estimate of the reduced axial force.

Furthermore, the predicted reaction stress is always approximately half the blood pressure smaller than in the corresponding in silico artery. An interesting observation is that by systematically overestimating the total circumferential stress by half the blood pressure, cf. $\alpha=0.5$ in Section 4.2.1, the erroneous reaction stress is compensated for. As a consequence, the isotropic and anisotropic stress components in the circumferential direction show a high agreement with the in silico arteries, see Figure 20a.

Since arteries are reported to grow and remodel themselves to equalize transmural gradients, cf. Section 2.2, their stress state appears to be adequately described by the thin-walled constitutive membrane model.

For detailed information about the comparison of the predicted and in silico stress states, the reader is referred to Paper II.

\subsection{Global optimization}

The minimization problem $(\mathbb{P I})$ is non-convex and to increase the likelihood that the global minimum is identified a heuristic method is used, cf. Chapter 6 . To ensure that indeed the global minimum is identified a deterministic global optimization algorithm based on the B\&B method introduced in Section 5.3 is created. The complete algorithm and the convex relaxation of $(\mathbb{P I})$ are found in Paper III.

The proposed B\&B algorithm is applied to three different clinical data sets taken from Sonesson et al. (1994). Despite parallelizing the B\&B algorithm and using a cluster with 108 cores as well as two personal computers, it is not feasible to solve the minimization problem to global $\tau$-optimality considering the whole parameter space given in Table 1. Instead a candidate global solution is determined for each clinical data set with the proposed heuristic method and the parameter space around these solutions is investigated. In total four increasingly larger, so called, vicinity regions are investigated for each data set, where the largest region is five times the $95 \%$ limits of agreement in Table 2 around the candidate global solution.

Solving the minimization problem with the largest vicinity region takes between $0.5-4$ months for each of the three clinical data sets. For each of the three clinical data sets and each vicinity region, the candidate global solution is identified during the initialization phase of the $\mathrm{B} \& \mathrm{~B}$ algorithm and a solution with a lower objective function value is never found. Hence, the same solution is obtained regardless of solving $(\mathbb{P I})$ to $\tau$-optimality using the $\mathrm{B} \& \mathrm{~B}$ algorithm or applying the proposed heuristic method.

For detailed information about the global optimization algorithm and the results, the reader is referred to Paper III. 


\subsection{Extension to account for smooth muscle activity}

The in vivo parameter identification method is extended to account for smooth muscle activity. For that purpose, the passive constitutive membrane model is replaced by the active constitutive membrane model, cf. Section 4.2 , and one additional parameter is identified for a pressure-radius data set, namely the generated isometric stress $S$ associated with the degree of smooth muscle activation. In order to avoid overparameterization, the active constitutive membrane model is fit simultaneously to three pressure-radius data sets of the same artery. These three data sets are collected at different levels of smooth muscle tone: basal during rest; constricted, induced by lower-body negative pressure; and dilated, induced by physical exercise. For each of the three pressure-radius data sets one unique model parameter $S_{q}$ is identified, where $q=1$ (basal), 2 (constricted), 3 (dilated). The parameters associated with the deformation $\left(R_{\mathrm{i}}, \lambda\right)$ and the passive response $\left(c, k_{1}, k_{2}, \beta\right)$ are the same for each of the three arterial conditions. Hence, in total nine model parameters are identified for an artery.

These nine model parameters are identified by minimizing the normalized sum of the individual objectives defined as

$$
\begin{array}{r}
\varepsilon_{q}^{\mathrm{act}}\left(\boldsymbol{\kappa}, S_{q}\right)=\sum_{j=1}^{n}\left\{w\left[\sigma_{\theta \theta}^{\mathrm{Lp}}\left(P_{j}, r_{\mathrm{i}, j}, h_{j}\right)-\sigma_{\theta \theta}^{\mathrm{act}}\left(\boldsymbol{\kappa}, S_{q}, r_{\mathrm{i}, j}, h_{j}\right)\right]^{2}+\ldots\right. \\
\left.(1-w)\left[\sigma_{z z}^{\mathrm{Lp}}\left(P_{j}, r_{\mathrm{i}, j}, h_{j}\right)-\sigma_{z z}^{\mathrm{act}}\left(\boldsymbol{\kappa}, r_{\mathrm{i}, j}, h_{j}\right)\right]^{2}\right\},
\end{array}
$$

where $w=0.99$, cf. Chapter 6 , and $\sigma_{\theta \theta}^{\mathrm{Lp}}, \sigma_{z z}^{\mathrm{Lp}}, \sigma_{\theta \theta}^{\text {act }}$, and $\sigma_{z z}^{\text {act }}$ are given by Eqs. $(76)$, (77), (85), and (86), respectively. In order to weigh each individual objective equally, i.e. each arterial condition, they are normalized using Utopia and Nadir points, cf. Normalization 2 in Section 5.1, and the active parameter identification problem reads:

$(\mathbb{A P I}) \begin{cases}\operatorname{minimize}_{(\boldsymbol{\kappa}) \in \mathbb{R}^{9}} & \sum_{q=1}^{3} \frac{\varepsilon_{q}^{\text {act }}\left(\boldsymbol{\kappa}, S_{q}\right)-\varepsilon_{q}^{\text {Utopia }}}{\varepsilon_{q}^{\text {Nadir }}-\varepsilon_{q}^{\text {Utopia }}}, \\ \text { subject to: } & \left\{\begin{array}{l}\boldsymbol{\kappa}^{\text {min }} \leq \boldsymbol{\kappa} \leq \boldsymbol{\kappa}^{\text {max }} \\ \boldsymbol{S}^{\text {min }} \leq \boldsymbol{S} \leq \boldsymbol{S}^{\text {max }},\end{array}\right.\end{cases}$

where $\boldsymbol{S}=\left(S_{1}, S_{2}, S_{3}\right)$, the box constraints for $\boldsymbol{\kappa}$ are given in Table 1, and $S_{1,2,3}^{\min }=$ $10^{-4} \mathrm{kPa}$ and $S_{1,2,3}^{\max }=150 \mathrm{kPa}$ are the lower and upper bound of $S$, respectively. The Utopia points $\varepsilon_{q}^{\text {Utopia }}$ in $(\mathbb{A P I})$ are determined using the passive constitutive membrane model, i.e. by solving $(\mathbb{P I})$ for each condition individually. This is done to obtain a unique solution which can be used to calculate the Nadir points $\varepsilon_{q}^{\text {Nadir }}$.

The minimization problem $(\mathbb{A P I})$ is similar to $(\mathbb{P I})$ and is solved in the same way: rescale the minimization problem according to Eq. (112); provide analytical expressions for gradient and Hessian of the objective function; and solve the minimization problem in MATLAB using the interior-point algorithm of fmincon with the proposed heuristic method and 100 starting points. 


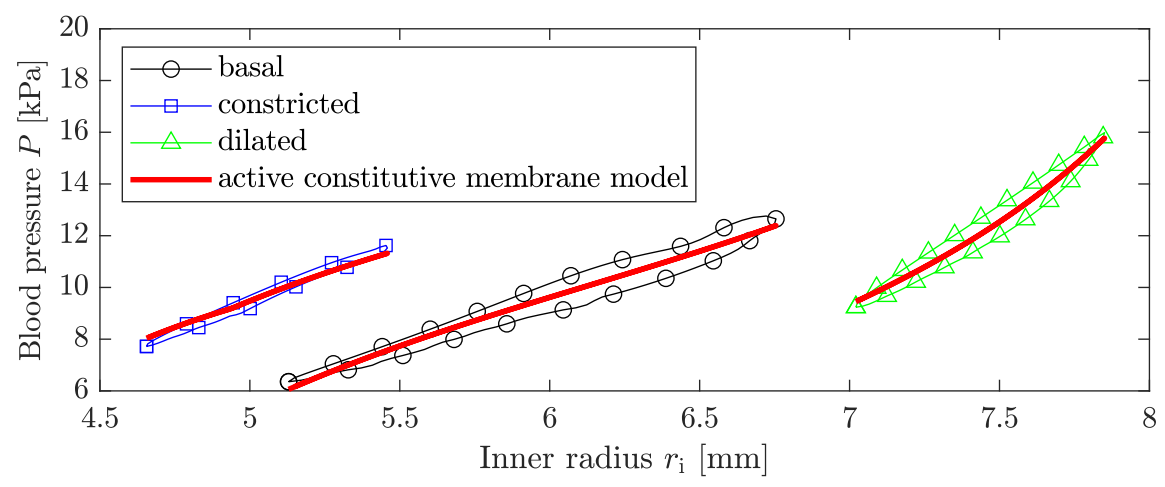

(a) Subject I, 24-year-old female

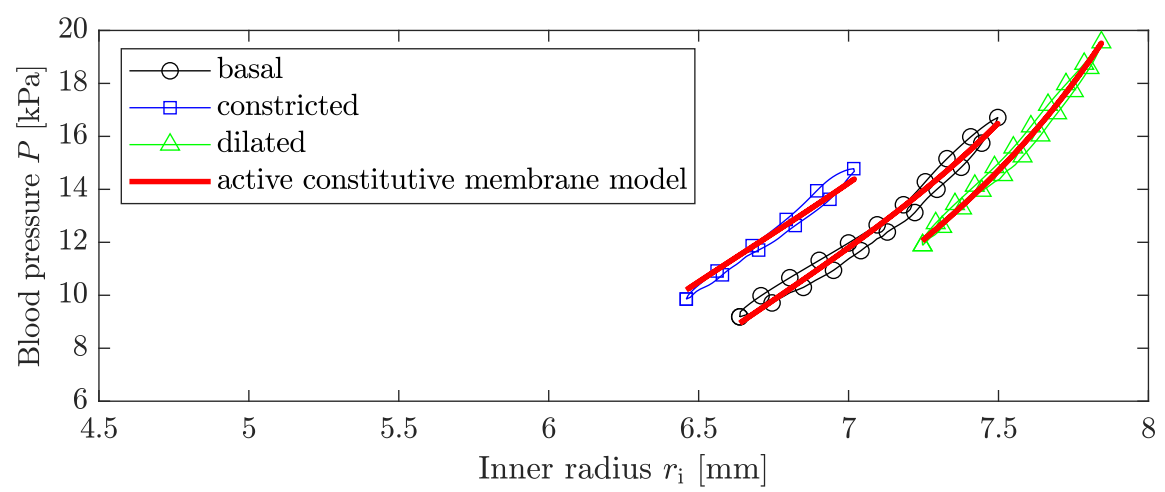

(b) Subject II, 26-year-old female

Figure 21: Measured pressure-radius curves and prediction of the active constitutive membrane model. Data is taken from Paper IV.

The mechanical properties considering smooth muscle activation are identified for the abdominal aorta of two female subjects taken from Sonesson et al. (1994, 1997). In both cases, the minimization problem is solved in less than a minute on a standard hexa-core computer, at least $98 \%$ of all starting points converge to the same solution with the lowest objective function value, and the Hessian has full rank at the solution.

In Figure 21 the clinical data measured during the three arterial conditions as well as the model prediction are visualized for both subjects. As can be seen, smooth muscle activity has an enormous effect on the mechanical response of an abdominal aorta and despite the simple smooth muscle model, the active constitutive membrane model is able to fit the pressure-radius curves well at rest, under lower-body negative pressure, and during physical exercise.

For detailed information, the reader is referred to Paper IV. 



\section{Discussion and Conclusion}

In this dissertation, a method is proposed that identifies the mechanical properties of an artery using clinical measurements. The mechanical properties of an artery are associated with the development of cardiovascular diseases and the possibility to identify the mechanical properties could, therefore, guide clinicians when diagnosing, treating, and monitoring diseases.

The proposed in vivo parameter identification method identifies the mechanical properties of an artery by fitting a continuum-mechanical model, the constitutive membrane model, to clinical data. The best-fit model parameters, which are related to the mechanical properties of the artery in question, are determined by solving a non-convex minimization problem stated in terms of the least-squares differences between the model's prediction and the clinical measurements. Since clinical data are generally limited to time-resolved blood pressure and inner radius of the arterial wall, the number of model parameters is restricted. Introducing too many parameters leads to overparameterization, meaning that not one set of model parameters describes the unique mechanical properties of the artery but that many parameter combinations characterize the vessel equally well. For that reason, the passive constitutive membrane model introduces only six model parameters and treats an artery as a homogeneous, incompressible, residual stress-free, and thin-walled tube consisting of an elastin dominated matrix with embedded collagen fibers.

The passive constitutive membrane model accounts for fewer arterial characteristics compared to the mechanical models used in other in vivo parameter identification methods. The models in other methods account for the thick-walled nature (Masson et al., 2008, Smoljkić et al., 2015, Spronck et al., 2015), real aortic geometry (Wittek et al., 2016), dispersion of collagen fibers (Smoljkić et al., 2015, Spronck et al., 2015, Wittek et al., 2016), smooth muscle activity (Masson et al., 2008, Spronck et al., 2015), and perivascular pressure (Masson et al., 2008). Only the mechanical model used in Schulze-Bauer and Holzapfel (2003) is similar to the constitutive membrane model but differs in the choice of the constitutive description.

All mentioned methods demonstrated the capability to determine reasonable mechanical properties of an artery for a few, or in case of the method proposed in Stålhand (2009), which the method proposed herein is based upon, for several, in vivo data sets. Unlike the method proposed herein however, none of them has undergone a proper validation ${ }^{1}$.

\footnotetext{
${ }^{1}$ The method presented in Smoljkić et al. (2015) has been validated, however, only using one in silico abdominal aorta of rat.
} 
The in vivo parameter identification method proposed in this dissertation is validated using 22 state-of-the-art finite element models using parameters corresponding to the human abdominal aorta from the literature. In this validation, see Paper I, it is shown, that despite the simplified passive constitutive membrane model, adequate model parameters are identified for healthy arteries and that pathological arteries are revealed from in vivo-like data (RQ1). In addition, the thin-walled mechanical model represents the stress state, even decomposed into an isotropic and anisotropic component, well for arteries with a physiological transmural stress gradient because arteries experience essentially a two-dimensional stress state in vivo (RQ2), see Paper II.

Another important aspect, common to all of the above-mentioned methods, is the identification of the model parameters by solving a non-convex minimization problem. While all of them use a more or less sophisticated heuristic method to solve the non-convex minimization problem, only for the in vivo parameter identification method proposed in this dissertation it is shown that indeed the global solution is obtained, see Paper III. Using the proposed global optimization algorithm to identify the mechanical properties of an artery appears to be impractical, however, especially since it is now known that the proposed heuristic method identifies the same solution in a fraction of the time (RQ3).

Lastly, the passive constitutive membrane model is extended by a smooth muscle contraction model which is similar to the ones used in Masson et al. (2008), Spronck et al. (2015). While the mechanical model in Masson et al. (2008) requires 14 parameters to be identified, making it very questionable whether a unique solution is obtained, Spronck et al. (2015) fix model parameters to reported values to prevent overparameterization. A different approach is chosen in this dissertation where the active constitutive membrane model is fit simultaneously to three pressure-radius data sets measured during different states of smooth muscle activity, see Paper IV. Not only is a unique solution obtained, but the active constitutive membrane model fits the clinical data measured at rest, under lower-body negative pressure, and during physical exercise well (RQ4). 


\section{Outlook}

An in vivo parameter identification method capable of identifying the mechanical properties of arteries is proposed in this dissertation and four important research questions are addressed. An overview of the research outcome is provided in Figure 22 .

Concerning the in vivo parameter identification method two aspects should be investigated further. First, the validation is based only on arterial-like finite element models. Although this validation approach provides a straight-forward possibility to compare the identified and pre-defined model parameters, as well as the decomposed stress state, this is only an intermediate step and eventually the method should be validated using in vitro experiments. Second, the method has only been used to identify the mechanical properties of healthy abdominal aortas and abdominal aortas with a pathologically small isotropic matrix contribution. Other arteries and especially arteries with different pathology should be investigated in the future.

Another open question is how the identified mechanical properties of an artery can be used in a clinical setting to guide clinicians when diagnosing, treating, and monitoring cardiovascular diseases.

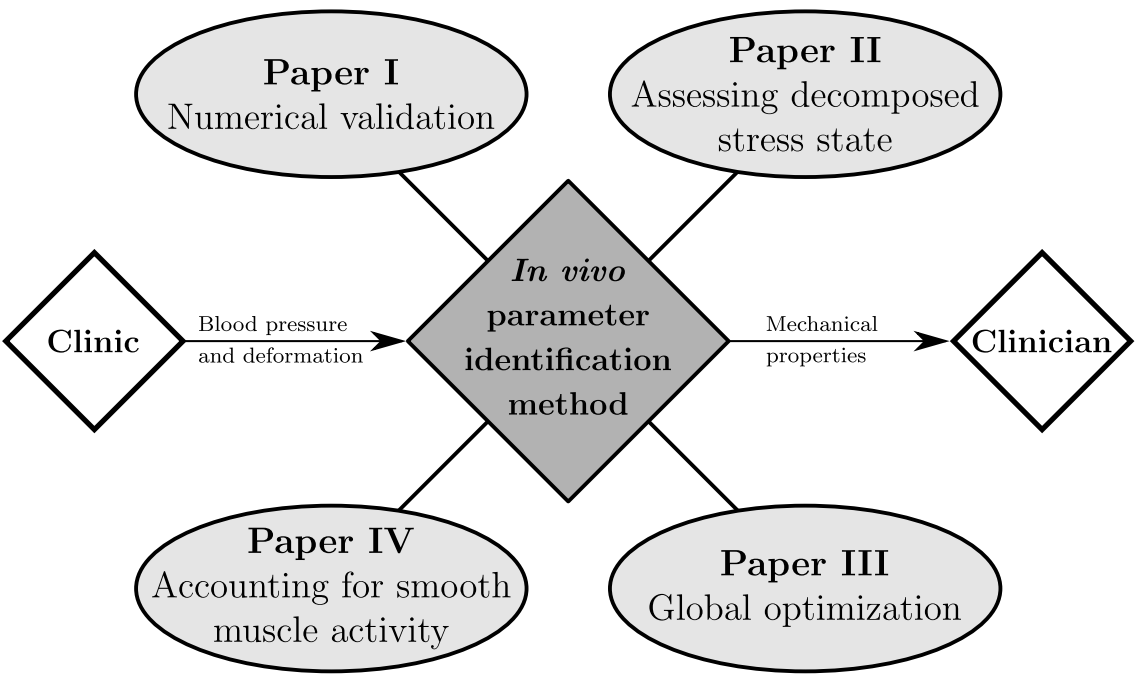

Figure 22: Overview of the Ph.D. project. 



\section{Review of Appended Papers}

\section{Paper I}

An in vivo parameter identification method for arteries: numerical validation for the human abdominal aorta

Review: An in vivo parameter identification method for arteries is proposed and validated for the human abdominal aorta. The parameter identification method determines the mechanical properties of an artery in terms of six relevant parameters based on in vivo measured pressure-radius data. To get insight into the accuracy of the method 22 arterial-like finite element models are created using published data for the human abdominal aorta. These in silico abdominal aortas serve as mock experiments with exactly known material properties and boundary conditions. Subsequently the in vivo parameter identification method is used to estimate the mechanical properties of the in silico arteries. By comparing the identified and pre-defined (material) parameters the method is quantitatively validated. The numerical validation indicates that the parameter identification method is able to identify adequate parameters for healthy abdominal aortas and reveals pathological aortas from in vivo-like data.

\section{Addressed research question:}

RQ1: How well does the proposed in vivo parameter identification method identify the mechanical properties of an artery?

\section{Paper II}

Assessing the accuracy of the stress predicted by an in vivo parameter identification method for arteries

Review: In this study, the stress state inside the arterial wall of the in silico arteries of Paper I is predicted. The stress state is decomposed into an isotropic and an anisotropic component which are associated with, primarily, the isotropic matrix and the collagen fibers, respectively. In order to assess the accuracy of the predicted stress state it is compared to the known stress state of the in silico arteries. The predicted decomposed stress state in the circumferential direction agrees well for 
arteries exhibiting a small (physiological) transmural stress gradient. In the axial direction larger discrepancies occur which is related to an erroneous estimate of the reduced axial force. The agreement in both the circumferential and the axial direction deteriorates with increasing transmural stress gradient and is related to the thin-walled assumption of the constitutive membrane model.

\section{Addressed research question:}

RQ2: How well does the arterial model used in the in vivo parameter identification method in combination with the identified model parameters represent the examined artery?

\section{Paper III}

Identification of mechanical properties of arteries with certification of global optimality

Review: The in vivo parameter identification method determines the mechanical properties of an artery by fitting the response of the constitutive membrane model to clinical data. The fitting of the model is formulated as a constrained non-linear, non-convex minimization problem. The non-convexity implies that incorrect model parameters, corresponding to local minima or stationary points may be found, however. A deterministic global optimization algorithm based on the B\&B method is, therefore, created and the minimization problem is solved to global optimality. The algorithm is tested on three clinical data sets. Despite dedicated hardware, it is not feasible to solve the minimization problem to global optimality considering the whole parameter space and four increasingly larger regions around a candidate global solution are considered instead. In all cases, the candidate global solution is found in the initialization phase of the $\mathrm{B} \& \mathrm{~B}$ algorithm and a better solution is never found. Although the $\mathrm{B} \& \mathrm{~B}$ algorithm is parallelized, solving the minimization problem to global optimality is very time-consuming.

\section{Addressed research question:}

RQ3: Is it feasible to employ deterministic global optimization methods to solve the minimization problem within the in vivo parameter identification method to obtain the global solution?

\section{Paper IV}

In vivo parameter identification in arteries considering multiple levels of smooth muscle activity

Review: In this study, the in vivo parameter identification method is extended to account for smooth muscle activity. Including smooth muscle activity into the 
constitutive membrane model increases the number of model parameters and, therefore, the risk of overparameterization. To prevent overparameterization, the active constitutive membrane model is fit simultaneously to clinical data measured at different levels of smooth muscle activity. Three arterial conditions are considered: basal during rest; constricted, induced by lower-body negative pressure; and dilated, induced by physical exercise. By fitting the active constitutive membrane model to these three arterial conditions simultaneously, overparameterization is prevented and a unique set of model parameters is obtained. The model prediction agrees well with the clinical data of two abdominal aortas.

\section{Addressed research question:}

RQ4: How can the arterial model be extended to account for smooth muscle activity without causing overparameterization? 



\section{References}

Adjiman CS, Androulakis IP, Floudas CA. 1998. A global optimization method, $\alpha \mathrm{BB}$, for general twice-differentiabe constrained NLPs-II. Implementation and computational results. Comput Chem Eng. 22(9):1159-1179.

Al-Khayyal FA. 1990. Jointly constrained bilinear programs and related problems: An overview. Comput Math with Appl. 19(11):53-62.

Arner A. 1982. Mechanical characteristics of chemically skinned guinea-pig taenia coli. Pflügers Arch Eur J Physiol. 395(4):277-284.

Atkinson CL, Carter HH, Naylor LH, Dawson EA, Marusic P, Hering D, Schlaich MP, Thijssen DH, Green DJ. 2015. Opposing effects of shear-mediated dilation and myogenic constriction on artery diameter in response to handgrip exercise in humans. J Appl Physiol. 119(8):858-864.

Auer M, Stollberger R, Regitnig P, Ebner F, Holzapfel GA. 2010. In vitro angioplasty of atherosclerotic human femoral arteries: Analysis of the geometrical changes in the individual tissues using mri and image processing. Ann Biomed Eng. 38(4):1276-1287.

Betts JT. 2010. Practical Methods for Optimal Control and Estimation Using Nonlinear Programming. 2nd ed.

Boudaoud S, Rix H, Meste O, Heneghan C, O'Brien C. 2007. Corrected Integral Shape Averaging Applied to Obstructive Sleep Apnea Detection from the Electrocardiogram. EURASIP J Adv Signal Process. 2007(1).

Bramwell JC, Hill AV. 1922. The Velocity of the Pulse Wave in Man. Proc R Soc B Biol Sci. 93(652):298-306.

Burton AC. 1954. Relation of Structure to Function of the Tissues of the Wall of Blood Vessels. Physiol Rev. 34(5):619-642.

Chadwick P. 1999. Continuum mechanics: concise theory and problems. 2nd ed. George Allen \& Unwin, Ltd.

Chaudhry HR, Bukiet B, Davis A, Ritter AB, Findley T. 1997. Residual stresses in oscillating thoracic arteries reduce circumferential stresses and stress gradients. J Biomech. 30(1):57-62. 
Choke E, Cockerill G, Wilson WRW, Sayed S, Dawson J, Loftus I, Thompson MM. 2005. A Review of Biological Factors Implicated in Abdominal Aortic Aneurysm Rupture. Eur J Vasc Endovasc Surg. 30(3):227-244.

Chuong CJ, Fung YC. 1986. On Residual Stresses in Arteries. J Biomech Eng. 108(2):189-192.

Cocciolone AJ, Hawes JZ, Staiculescu MC, Johnson EO, Murshed M, Wagenseil JE. 2018. Elastin, arterial mechanics, and cardiovascular disease. Am J Physiol - Hear Circ Physiol. 315(2):H189-H205.

Conroy RM, Pyörälä K, Fitzgerald AP, Sans S, Menotti A, De Backer G, De Bacquer D, Ducimetière P, Jousilahti P, Keil U, et al. 2003. Estimation of tenyear risk of fatal cardiovascular disease in Europe: The SCORE project. Eur Heart J. 24(11):987-1003.

Cox RH. 1978a. Comparison of carotid artery mechanics in the rat, rabbit, and dog. Am J Physiol - Hear Circ Physiol. 3(3):280-288.

Cox RH. 1978b. Regional Variation of Series Elasticity in Canine Arterial Smooth Muscles. Am J Physiol. 234(18):H542-H551.

Curry SJ, Krist AH, Owens DK, Barry MJ, Caughey AB, Davidson KW, Doubeni CA, Epling JW, Kemper AR, Kubik M, et al. 2018. Risk assessment for cardiovascular disease with nontraditional risk factors: US preventive services task force recommendation statement. JAMA - J Am Med Assoc. 320(3):272-280.

D'Agostino RB, Vasan RS, Pencina MJ, Wolf PA, Cobain M, Massaro JM, Kannel WB. 2008. General cardiovascular risk profile for use in primary care: The Framingham heart study. Circulation. 117(6):743-753.

De Keulenaer BL, De Waele JJ, Powell B, Malbrain ML. 2009. What is normal intra-abdominal pressure and how is it affected by positioning, body mass and positive end-expiratory pressure? Intensive Care Med. 35(6):969-976.

Demiray H, Vito RP. 1983. On Large Periodic Motions of Arteries. J Biomech. 16(8):643-648.

Dillon PF, Aksoy MO, Driska SP, Murphy RA. 1981. Myosin Phosphorylation and the Cross-Bridge Cycle in Arterial Smooth Muscle. Science. 211(4481):495-497.

Dobrin PB. 1973. Influence of initial length on length tension relationship of vascular smooth muscle. Am J Physiol. 225(3):664-670.

Dobrin PB. 2011. Vascular Mechanics. In: Compr. physiol. p. 65-102.

Ecobici M, Stoicescu C. 2017. Arterial Stiffness and Hypertension - Which Comes First? Maedica (Buchar). 12(3):184-190. 
Esposito WR, Floudas CA. 1998. Global optimization in parameter estimation of nonlinear algebraic models via the error-in-variables approach. Ind Eng Chem Res. 37(5):1841-1858.

Ferruzzi J, Vorp DA, Humphrey JD. 2011. On Constitutive Descriptors of the Biaxial Mechanical Behaviour of Human Abdominal Aorta and Aneurysms. J R Soc Interface. 8(56):435-450.

Fletcher R. 2000. Practical Methods of Optimization. John Wiley \& Sons, Ltd.

Frank O. 1990. The basic shape of the arterial pulse. First treatise: Mathematical analysis. J Mol Cell Cardiol. 22(3):255-277.

Fung YC. 1991. What are the residual stresses doing in our blood vessels? Ann Biomed Eng. 19(3):237-249.

Fung YC. 1993. Biomechanics: Mechanical Properties of Living Tissues. 2nd ed. Springer-Verlag New York.

Fung YC, Fronek K, Patitucci P. 1979. Pseudoelasticity of arteries and the choice of its mathematical expression. Am J Physiol. 237(5):H620-H631.

Fung YC, Liu SQ. 1989. Change of Residual Strains in Arteries due to Hypertrophy Caused by Aortic Constriction. Circ Res. 65(5):1340-1349.

Gade JL. 2019. Mechanical properties of arteries: Identification and Application. Linköping Stud Sci Technol Licent Thesis. (1849).

Gasser TC, Ogden RW, Holzapfel GA. 2006. Hyperelastic Modelling of Arterial Layers with Distributed Collagen Fibre Orientations. J R Soc Interface. 3(6):1535.

Gasser TC, Schulze-Bauer CAJ, Holzapfel GA. 2002. A Three-Dimensional Finite Element Model for Arterial Clamping. J Biomech Eng. 124(4):355-363.

Green DJ, Hopman MT, Padilla J, Laughlin MH, Thijssen DH. 2017. Vascular adaptation to exercise in humans: Role of hemodynamic stimuli. Physiol Rev. 97(2):495-528.

Greenwald SE, Moore JE, Rachev A, Kane TP, Meister JJ. 1997. Experimental Investigation of the Distribution of Residual Strains in the Artery Wall. J Biomech Eng. 119(4):438-444.

Gundiah N, B Ratcliffe M, A Pruitt L. 2007. Determination of strain energy function for arterial elastin: Experiments using histology and mechanical tests. J Biomech. 40(3):586-594.

Gundiah N, Ratcliffe MB, Pruitt LA. 2009. The biomechanics of arterial elastin. J Mech Behav Biomed Mater. 2(3):288-296. 
Gurtin ME. 1981. An introduction to continuum mechanics. Academic Press.

Guyton AC, Hall JE. 2006. Textbook of Medical Physiology. 11th ed. Elsevier Saunders.

Hai CM, Murphy RA. 1988. Cross-bridge phosphorylation and regulation of latch state in smooth muscle. Am J Physiol Physiol. 254(1):C99-C106.

Han HC, Fung YC. 1996. Direct Measurement Strains in Aorta of Transverse Residual. Am J Physiol. 270(2):H750-H759.

Hill AV. 1938. The heat of shortening and the dynamic constants of muscle. Proc R Soc London Ser B - Biol Sci. 126(843):136-195.

Holzapfel GA. 2000. Nonlinear Solid Mechanics: A Continuum Approach for Engineering. Chichester: John Wiley, cop. 2000.

Holzapfel GA, Gasser TC, Ogden RW. 2000. A New Constitutive Framework for Arterial Wall Mechanics and a Comperative Study of Material Models. J Elast. 61(1):1-48.

Holzapfel GA, Niestrawska JA, Ogden RW, Reinisch AJ, Schriefl AJ. 2015. Modelling Non-Symmetric Collagen Fibre Dispersion in Arterial Walls. J R Soc Interface. $3(6): 15-35$.

Holzapfel GA, Ogden RW. 2015. On the Tension - Compression Switch in Soft Fibrous Solids. Eur J Mech / A Solids. 49:561-569.

Holzapfel GA, Sommer G, Auer M, Regitnig P, Ogden RW. 2007. Layer-Specific 3D Residual Deformations of Human Aortas with Non-Atherosclerotic Intimal Thickening. Ann Biomed Eng. 35(4):530-545.

Horný L, Adámek T, Gultova E, Zitny R, Vesely J, Chlup H, Konvickova S. 2011. Correlations Between Age, Prestrain, Diameter and Atherosclerosis in the Male Abdominal Aorta. J Mech Behav Biomed Mater. 4(8):2128-2132.

Horst R, Tuy H. 1996. Global Optimization: Deterministic Approaches. 3rd ed. Springer-Verlag Berlin Heidelberg.

Humphrey JD. 2002. Cardiovascular Solid Mechanics. 1st ed. Springer-Verlag New York.

Humphrey JD, Na S. 2002. Elastodynamics and arterial wall stress. Ann Biomed Eng. 30(4):509-523.

Humphrey JD, Wilson E. 2003. A potential role of smooth muscle tone in early hypertension: A theoretical study. J Biomech. 36(11):1595-1601.

Huxley AF, Niedergerke R. 1954. Structural changes in muscle during contraction. Nature. 173(4412):971-973. 
Huxley H, Hanson J. 1954. Changes in the Cross-Striations of Muscle during Contraction and Stretch and their Structural Interpretation. Nature. 173(4412):973-976.

Karush W. 2014. Minima of functions of several variables with inequalities as side conditions. In: Traces emerg. nonlinear program.

Kawasaki T, Sasayama S, Yagi SI, Asakawa T, Hirai T. 1987. Non-Invasive Assessment of the Age Related Changes in Stiffness of Major Branches of the Human Arteries. Cardiovasc Res. 21(9):678-687.

Kim J, Peruski B, Hunley C, Kwon S, Baek S. 2013. Influence of Surrounding Tissues on Biomechanics of Aortic Wall. Int J Exp Comput Biomech. 2(2):105-117.

Kroon M. 2009. A constitutive model for smooth muscle including active tone and passive viscoelastic behaviour. Math Med Biol. 27(2):129-155.

Kuhn HW, Tucker AW. 2014. Nonlinear programming. In: Traces emerg. nonlinear program.

Labrosse MR, Gerson ER, Veinot JP, Beller CJ. 2013. Mechanical Characterization of Human Aortas from Pressurization Testing and a Paradigm Shift for Circumferential Residual Stress. J Mech Behav Biomed Mater. 17:44-55.

Laksari K, Shahmirzadi D, Acosta CJ, Konofagou E. 2016. Energy-based constitutive modelling of local material properties of canine aortas. R Soc Open Sci. 3(9):1-10.

Laurent S, Alivon M, Beaussier H, Boutouyrie PH. 2012. Aortic stiffness as a tissue biomarker for predicting future cardiovascular events in asymptomatic hypertensive subjects. Ann Med. 44(SUPPL. 1).

Laurent S, Boutouyrie PH, Lacolley P. 2005. Structural and Genetic Bases of Arterial Stiffness. Hypertension. 45(6):1050-1055.

Laurent S, Cockcroft J, Van Bortel LM, Boutouyrie PH, Giannattasio C, Hayoz D, Pannier B, Vlachopoulos C, Wilkinson I, Struijker-Boudier HAJ. 2006. Expert Consensus Document on Arterial Stiffness: Methodological Issues and Clinical Applications. Eur Heart J. 27(21):2588-2605.

Learoyd BM, Taylor MG. 1966. Alterations with age in the viscoelastic properties of human arterial walls. Circ Res. 18(3):278-292.

Mahnken R. 2004. Identification of Material Parameters for Constitutive Equations. American Cancer Society. chap. 19.

Mancia G, De Backer G, Dominiczak A, Cifkova R, Fagard R, Germano G, Grassi G, Heagerty AM, Kjeldsen SE, Laurent S, et al. 2007. 2007 Guidelines for the Management of Arterial Hypertension. Eur Heart J. 28(12):1462-1536.

Maranas CD, Floudas CA. 1994. Global minimum potential energy conformations of small molecules. J Glob Optim. 4(2):135-170. 
Maranas CD, Floudas CA. 1995. Finding all solutions of nonlinearly constrained systems of equations. J Glob Optim. 7(2):143-182.

Masson I, Boutouyrie PH, Laurent S, Humphrey JD, Zidi M. 2008. Characterization of Arterial Wall Mechanical Behavior and Stresses from Human Clinical Data. J Biomech. 41(12):2618-2627.

MATLAB. 2020. Optimization Toolbox: User's Guide.

Matsumoto T, Goto T, Furukawa T, Sato M. 2004. Residual stress and strain in the lamellar unit of the porcine aorta: Experiment and analysis. J Biomech. $37(6): 807-815$.

Mausser H. 2006. Normalization and Other Topics in Multi-Objective Optimization. In: Proceeding Fields-MITACS Ind. Probl. Work. p. 89-101.

McCormick GP. 1976. Computability of global solutions to factorable nonconvex programs: Part I - Convex underestimating problems. Math Program. 10:147-175.

McKay MD, Beckman RJ, Conover WJ. 2000. A comparison of three methods for selecting values of input variables in the analysis of output from a computer code. Technometrics. 42(1):55-61.

Mozaffarian D, Benjamin EJ, Go AS, Arnett DK, Blaha MJ, Cushman M, Das SR, de Ferranti S, Després JP, Fullerton HJ, et al. 2016. Heart Disease and Stroke Statistics - 2016 Update: A Report From the American Heart Association. vol. 133.

Murphy RA. 1982. Myosin Phosphorylation and Crossbridge regulation in Arterial Smooth Muscle: State-of-the-art review. Hypertension. 4(3):3-7.

Murtada SI, Arner A, Holzapfel GA. 2012. Experiments and mechanochemical modeling of smooth muscle contraction: Significance of filament overlap. J Theor Biol. 297:176-186.

Murtada SI, Kroon M, Holzapfel GA. 2010. A calcium-driven mechanochemical model for prediction of force generation in smooth muscle. Biomech Model Mechanobiol. 9(6):749-762.

Najman J, Bongartz D, Mitsos A. 2019. Convex relaxations of componentwise convex functions. Comput Chem Eng. 130.

Nichols WW, O'Rourke MF. 2005. McDonald's Blood Flow in Arteries Theoretical, Experimental and Clinical Principles. 5th ed. London : New York, NY : Hodder Arnold ; Distributed in the United States of America by Oxford University Press, 2005 .

Nocedal J, Wright SJ. 1999. Numerical Optimization. 2nd ed. New York: Springer. 
O'Rourke MF. 1990. Arterial stiffness, systolic blood pressure, and logical treatment of arterial hypertension. Hypertension. 15(4):339-347.

Peterson LH, Jensen RE, Parnell J. 1960. Mechanical Properties of Arteries in Vivo. Circ Res. 8(3):622-639.

Price JM, Davis DL, Knauss EB. 1981. Length-dependent sensitivity in vascular smooth muscle. Am J Physiol - Hear Circ Physiol. 10(4):557-563.

Rachev A, Hayashi K. 1999. Theoretical Study of the Effects of Vascular Smooth Muscle Contraction on Strain and Stress Distributions in Arteries. Ann Biomed Eng. 27(4):459-468.

Reesink KD, Spronck B. 2019. Constitutive interpretation of arterial stiffness in clinical studies: A methodological review. Am J Physiol - Hear Circ Physiol. 316(3):H693-H709.

Rhodin JAG. 2014. Architecture of the Vessel Wall. In: Compr. physiol. American Cancer Society; chap. 1; p. 1-31.

Roach MR, Burton AC. 1957. The Reason for the Shape of the Distensibility Curves of Arteries. Can J Biochem Physiol. 35(8):681-690.

Rodbard S. 1975. Vascular Caliber. Cardiology. 60:4-49.

Roy CS. 1881. The Elastic Properties of the Arterial Wall. J Physiol. 3(2):125-159.

Saini A, Berry C, Greenwald SE. 1995. Effect of Age and Sex on Residual Stress in the Aorta. J Vasc Res. 32(6):398-405.

Schmitz A, Böl M. 2011. On a phenomenological model for active smooth muscle contraction. J Biomech. 44(11):2090-2095.

Schriefl AJ, Schmidt T, Balzani D, Sommer G, Holzapfel GA. 2015. Selective enzymatic removal of elastin and collagen from human abdominal aortas: Uniaxial mechanical response and constitutive modeling. Acta Biomater. 17:125-136.

Schriefl AJ, Zeindlinger G, Pierce DM, Regitnig P, Holzapfel GA. 2012. Determination of the layer-specific distributed collagen fibre orientations in human thoracic and abdominal aortas and common iliac arteries. J R Soc Interface. 9(71):1275-1286.

Schulze-Bauer CAJ, Holzapfel GA. 2003. Determination of Constitutive Equations for Human Arteries from Clinical Data. J Biomech. 36(2):165-169.

Schulze-Bauer CAJ, Mörth C, Holzapfel GA. 2003. Passive Biaxial Mechanical Response of Aged Human Iliac Arteries. J Biomech Eng. 125(3):395-406. 
Schulze-Bauer CAJ, Regitnig P, Holzapfel GA. 2002. Mechanics of the human femoral adventitia including the high-pressure response. Am J Physiol Circ Physiol. 282(6):H2427-H2440.

Singh SI, Devi LS. 1990. A Study on Large Radial Motion of Arteries In Vivo. J Biomech. 23(11):1087-1091.

Smoljkić M, Vander Sloten J, Segers P, Famaey N. 2015. Non-Invasive, Energy-Based Assessment of Patient-Specific Material Properties of Arterial Tissue. Biomech Model Mechanobiol. 14(5):1045-1056.

Somlyo AP, Somlyo AV. 1992. Smooth Muscle Structure and Function. In: Fozzard HA, editor. Hear. cardiovasc. syst. 2nd ed. Raven Press, Ltd., New York; chap. 48; p. 1295-1324.

Sonesson B, Länne T, Vernersson E, Hansen F. 1994. Sex difference in the mechanical properties of the abdominal aorta in human beings. J Vasc Surg. 20(6):959-969.

Sonesson B, Vernersson E, Hansen F, Länne T. 1997. Influence of Sympathetic Stimulation on the Mechanical Properties of the Aorta in Humans. Acta Physiol Scand. 159(2):139-145.

Spencer AJ. 1984. Constitutive Theroy for Strongly Anisotropic Solids.

Spronck B, Heusinkveld MH, Donders WP, De Lepper AG, Roodt JO, Kroon AA, Delhaas T, Reesink KD. 2015. A constitutive modeling interpretation of the relationship among carotid artery stiffness, blood pressure, and age in hypertensive subjects. Am J Physiol - Hear Circ Physiol. 308(6):H568-H582.

Stålhand J. 2009. Determination of Human Arterial Wall Parameters from Clinical Data. Biomech Model Mechanobiol. 8(2):141-148.

Stålhand J, Klarbring A. 2005. Aorta In Vivo Parameter Identification Using an Axial Force Constraint. Biomech Model Mechanobiol. 3(4):191-199.

Stålhand J, Klarbring A, Holzapfel GA. 2011. A mechanochemical 3D continuum model for smooth muscle contraction under finite strains. J Theor Biol. 268(1):120130.

Stålhand J, Klarbring A, Karlsson M. 2004. Towards in vivo aorta material identification and stress estimation. Biomech Model Mechanobiol. 2(3):169-186.

Taber LA, Humphrey JD. 2002. Stress-Modulated Growth, Residual Stress, and Vascular Heterogeneity. J Biomech Eng. 123(6):528.

Tadmor EB, Miller RE, Elliott RS. 2011. Continuum mechanics and thermodynamics: From fundamental concepts to governing equations. Cambridge: Cambridge University Press. 
Takamizawa K, Hayashi K. 1987. Strain Energy Density Function and Uniform Strain Hypothesis for Arterial Mechanics. J Biomech. 20(1):7-17.

Takamizawa K, Hayashi K, Matsuda T. 1992. Isometric biaxial tension of smooth muscle in isolated cylindrical segments of rabbit arteries. Am J Physiol - Hear Circ Physiol. 263(1 32-1):30-34.

Tortora GJ, Derrickson B. 2012. Prinicples of anatomy \& physiology. 13th ed. John Wiley \& Sons, Inc.

Treloar LRG. 1943. The elasticity of a network of long-chain molecules-II. Trans Faraday Soc. 39(0):241-246.

Truesdell C, Noll W. 1992. The Non-Linear Field Theories of Mechanics.

Tsamis A, Krawiec JT, Vorp DA. 2013. Elastin and Collagen Fibre Microstructure of the Human Aorta in Ageing and Disease: a Review. J R Soc Interface. 10(83):20121004.

Ugural AC. 1999. Stresses in plates and shells. 2nd ed. New York, London: McGrawHill.

Van Loon P, Klip W, Bradley EL. 1977. Length-Force and Volume-Pressure Relationships of Arteries. Biorheology. 14(4):181-201.

Van Sloten TT, Schram MT, Van Den Hurk K, Dekker JM, Nijpels G, Henry RM, Stehouwer CD. 2014. Local stiffness of the carotid and femoral artery is associated with incident cardiovascular events and all-cause mortality: The hoorn study. J Am Coll Cardiol. 63(17):1739-1747.

Vlachopoulos C, Xaplanteris P, Aboyans V, Brodmann M, Cífková R, Cosentino F, De Carlo M, Gallino A, Landmesser U, Laurent S, et al. 2015. The role of vascular biomarkers for primary and secondary prevention. A position paper from the European Society of Cardiology Working Group on peripheral circulation. Endorsed by the Association for Research into Arterial Structure and Physiology (ARTERY. Atherosclerosis. 241(2):507-532.

Volokh KY. 2006. Lagrangian equilibrium equations in cylindrical and spherical coordinates. Comput Mater Contin. 3(1):37-42.

Vorp DA. 2007. Biomechanics of Abdominal Aortic Aneurysm. J Biomech. 40(9):1887-1902.

Vossoughi J, Hedjazi Z, Borris FS. 1993. Intimal Residual Stress and Strain in Large Arteries. In: ASME Bioeng. Conf.; vol. 24; New York. ASME. p. 434-437.

Wächter A, Biegler LT. 2006. On the implementation of an interior-point filter line-search algorithm for large-scale nonlinear programming. Math Program. 106(1):25-57. 
Weizsäcker HW, Lambert H, Pascale K. 1983. Analysis of the Passive Mechanical Properties of Rat Carotid Arteries. J Biomech. 16(9):703-715.

Wilkins E, Wilson L, Wickramasinghe K, Bhatnagar P, Leal J, Luengo-Fernandez R, Burns R, Rayner M, Townsend N. 2017. European Cardiovascular Disease Statistics 2017. Eur Hear Network, Brussels:192.

Wittek A, Derwich W, Karatolios K, Fritzen P, Vogt S, Schmitz-Rixen T, Blase C. 2016. A Finite Element Updating Approach for Identification of the Anisotropic Hyperelastic Properties of Normal and Diseased Aortic Walls from 4D Ultrasound Strain Imaging. J Mech Behav Biomed Mater. 58:122-138.

Yosibash Z, Manor I, Gilad I, Willentz U. 2014. Experimental evidence of the compressibility of arteries. J Mech Behav Biomed Mater. 39:339-354.

Zamora JM, Grossmann IE. 1999. A Branch and Contract Algorithm for Problems with Concave Univariate, Bilinear and Linear Fractional Terms. J Glob Optim. 14(3):217-249.

Zeller PJ, Skalak TC. 1998. Contribution of individual structural components in determining the zero-stress state in small arteries. J Vasc Res. 35(1):8-17.

Zieff GH, Heffernan K, Stone K, Fryer S, Credeur D, Hanson ED, Faulkner J, Stoner L, Article O. 2019. The pressure-dependency of local measures of arterial stiffness. J Hypertens. 37(5):956-963.

Zieman SJ, Melenovsky V, Kass DA. 2005. Mechanisms, pathophysiology, and therapy of arterial stiffness. Arterioscler Thromb Vasc Biol. 25(5):932-943.

Zulliger MA, Rachev A, Stergiopulos N. 2004. A constitutive formulation of arterial mechanics including vascular smooth muscle tone. Am J Physiol - Hear Circ Physiol. 287(3 56-3):1335-1343. 
Part II

Appended Papers 


\section{Papers}

The papers associated with this thesis have been removed for copyright reasons. For more details about these see:

http://urn.kb.se/resolve?urn=urn:nbn:se:liu:diva-172297 


\section{FACULTY OF SCIENCE AND ENGINEERING}

Linköping Studies in Science and Technology. Dissertations, No. 2113, 2021

Solid Mechanics, Department of Management and Engineering

Linköping University

SE-581 83 Linköping, Sweden

www.liu.se 